\title{
Twinning, a promising dynamic process to strengthen the agency of midwives
}

Citation for published version (APA):

Cadee, F. (2020). Twinning, a promising dynamic process to strengthen the agency of midwives. [Doctoral Thesis, Maastricht University]. Maastricht University. https://doi.org/10.26481/dis.20201009fc

Document status and date:

Published: 01/01/2020

DOI:

10.26481/dis.20201009fc

Document Version:

Publisher's PDF, also known as Version of record

\section{Please check the document version of this publication:}

- A submitted manuscript is the version of the article upon submission and before peer-review. There can be important differences between the submitted version and the official published version of record.

People interested in the research are advised to contact the author for the final version of the publication, or visit the DOI to the publisher's website.

- The final author version and the galley proof are versions of the publication after peer review.

- The final published version features the final layout of the paper including the volume, issue and page numbers.

Link to publication

\footnotetext{
General rights rights.

- You may freely distribute the URL identifying the publication in the public portal. please follow below link for the End User Agreement:

www.umlib.nl/taverne-license

Take down policy

If you believe that this document breaches copyright please contact us at:

repository@maastrichtuniversity.nl

providing details and we will investigate your claim.
}

Copyright and moral rights for the publications made accessible in the public portal are retained by the authors and/or other copyright owners and it is a condition of accessing publications that users recognise and abide by the legal requirements associated with these

- Users may download and print one copy of any publication from the public portal for the purpose of private study or research.

- You may not further distribute the material or use it for any profit-making activity or commercial gain

If the publication is distributed under the terms of Article $25 \mathrm{fa}$ of the Dutch Copyright Act, indicated by the "Taverne" license above, 


\section{Twinning, a promising dynamic process to strengthen the agency of midwives}

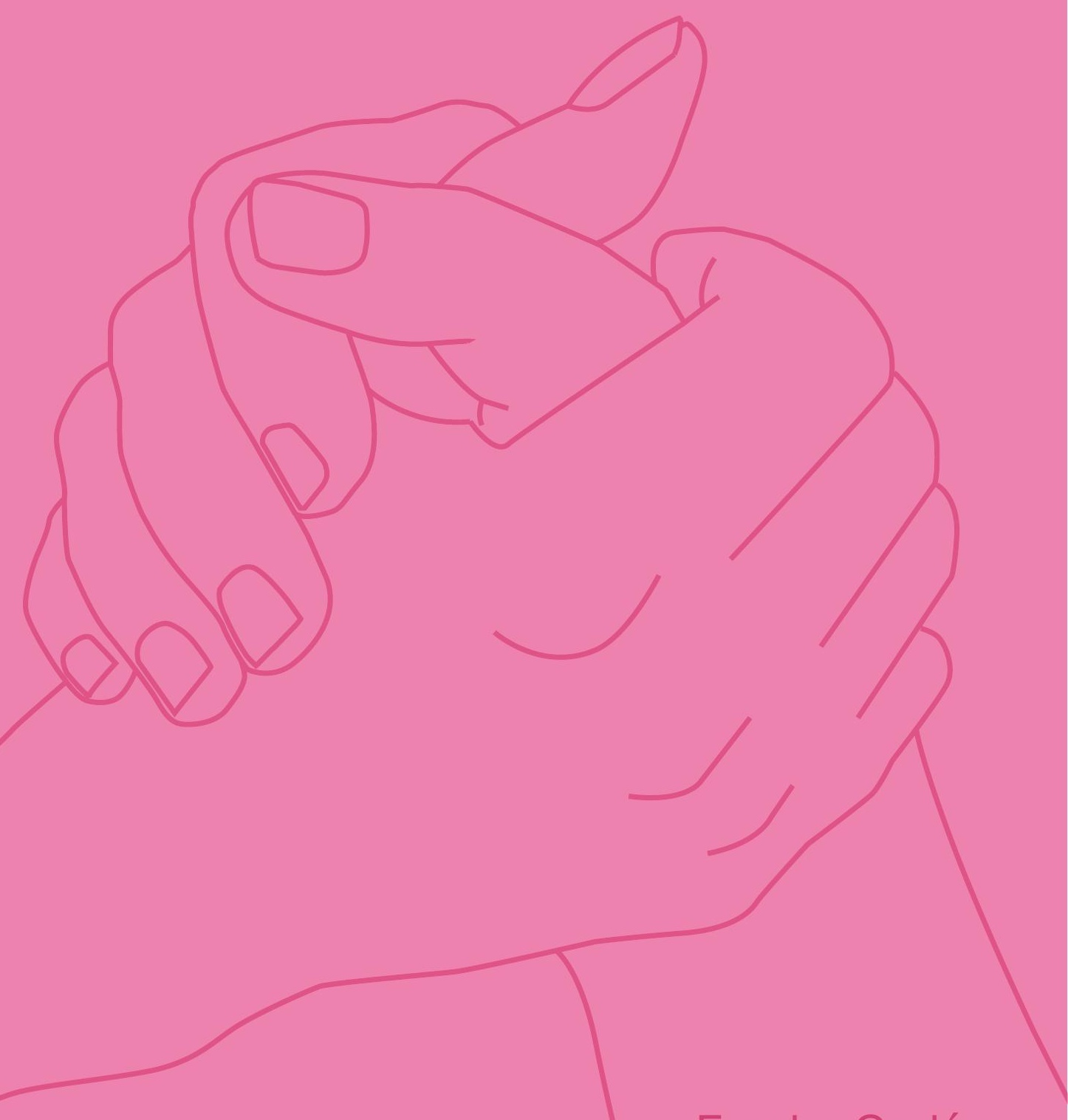

Franka Cadée 
Twinning, a promising dynamic process

to strengthen the agency of midwives 
Twinning, a promising dynamic process

to strengthen the agency of midwives

Thesis

To obtain the degree of Doctor at Maastricht University

On the authority of the Rector Magnificus, Prof. Dr. Rianne.M.Letchert

In accordance with the decision of the Board of Deans

To be defended in public on $9^{\text {th }}$ October 2020, 10:00

Twinning, a promosing dynamic process to strengthen the agency of midwives

\section{ISBN: $\quad$ 978-94-6402-165-3 \\ Cover: $\quad$ Ella Cadée \\ Lay-out: Ilse Modder | www.ilsemodder.nl \\ Printing: $\quad$ Gildeprint B.V. | www.gildeprint.nl}

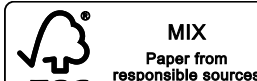

Copyright @ F. Cadée, 2020

All rights reserved. No part of this thesis may be reproduced, stored in a retrieval system,

or transmitted in any form or by any means, electronically, mechanically, by photocopy, by

recording, or otherwise, without prior written permission of the author.
By

Franka Cadée

Date of Birth: $16^{\text {th }}$ January 1963 
Supervisors

Prof. Dr. Raymond de Vries

Prof. Dr. Antoine L.M Lagro-Janssen

Co-supervisor

Prof. Dr. Marianne J Nieuwenhuijze

Assessment committee Prof. Dr. A. Krumeich

Prof. Dr. K. Horstman

Prof. Dr. E. van Teijlingen

Dr. F. Mevissen

Dr. M. Rijnders
I dedicate this work to midwives globally to encourage them to speak up and be heard

\section{I gratefully acknowledge the following organisations for their financial support}

The charity midwives 4 mothers www.midwives4mothers.nl enabled me to outsource some of the focus-group transcriptions and fund the travel and accommodation necessary for our third study (chapter four).

The Stichting Bijzondere Voorzieningen Moederschapszorg, Maastricht, the Netherlands (Charity for special opportunities for Motherhood-care) enabled me to dedicate analysis and writing time to our fourth study (chapter five).

The Royal Dutch NL of Midwives (KNOV) and Maastricht University supported the publication of this thesis as well as some of the celebratory festivities.

I received no other funding from funding agencies in the public, commercial, or not-for-profit sector. 


\section{CONTENT}

Chapter 1 General Introduction. Twinning, a promising dynamic process to strengthen the agency of midwives. Franka Cadée, Marianne J. Nieuwenhuijze, A. L. M. Lagro-Janssen and Raymond De Vries.

Chapter 2

The state of the art of twinning. A concept analysis of twinning in healthcare.

Franka Cadée, Marianne J. Nieuwenhuijze, Antoine L. M. Lagro-Janssen and Raymond De Vries.

Globalization and Health (2016) 12:66 DOI 10.1186/s12992-016-0205-5.

Chapter 3

From equity to power: Critical success factors for

twinning between midwives, a Delphi study.

Franka Cadée, Marianne J. Nieuwenhuïze, Antoine L. M. Lagro-Janssen and Raymond De Vries.

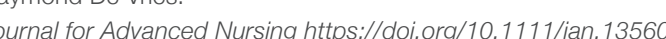

Chapter 4

Embrace the complex dynamics of twinning!

Franka Cadée, Marianne J. Nieuwenhuijze, Antoine L. M. Lagro-Janssen and Raymond De Vries.

Sage Open re-submitted Dec. 2019 (minor changes).

Chapter $5 \quad$ Paving the way for successful twinning. Using grounded theory to understand the contribution of twin pairs in twinning collaborations.

Franka Cadée, Marianne J. Nieuwenhuijze, Antoine L. M. Lagro-Janssen and Raymond De Vries.

Women and Birth https://doi.org/10.1016/j.wombi.2020.01.013.

Chapter 6 General Discussion. The complexity of twinning in

the field.

Franka Cadée, Marianne J. Nieuwenhuijze, Antoine L. M. Lagro-Janssen and Raymond De Vries.

Chapter 7

English summary of this thesis.

Chapter 8

Nederlandse samenvatting van dit proefschrift.

Chapter 9

Valorisation.

Chapter 10 Acknowledgements.

Chapter 11 Curriculum Vitea. 


\section{INTRODUCTION}

The Sustainable Development Goals (SDG), adopted by the United Nations (UN) in 2015, are a "blueprint to achieve a better and more sustainable future for all" "1]. The UN is committed to achieving these 17 goals - addressing a wide range of global challenges - by the year 2030. SDG 3 and SDG 5 are particularly relevant for those who provide maternity care [2, 3]. SDG 3 aims for a reduction in the global maternal mortality ratio to less than 70 per 100,000 live births by 2030; SDG 5 seeks to achieve gender equality and to empower all women and girls. Furthermore, as part of the SDGs, all United Nations Member States have agreed to try to achieve Universal Health Coverage (UHC), that is, having the full spectrum of quality health services - including health promotion and prevention, treatment, rehabilitation, and palliative care - available to all, without imposing financial hardship ${ }^{[4]}$. Women agree with these goals. A survey of "what women want" - done by the White Ribbon Alliance and including responses from more than 1,2 million women around the world - clearly shows that women and girls globally are urgently demanding their right to optimal sexual, reproductive and maternal healthcare ${ }^{[5]}$. The words of Betsy McCallon, CEO of White Ribbon Alliance, say it well: "If we do not listen and act on these demands we have only ourselves to blame for stalled progress." ${ }^{[5]}$.

Universal access to quality midwifery care is an effective way to respond to the demands of women for optimal care and, at the same time, it is the most direct path for achieving Sustainable Development Goals 3 and 5 . We know that the provision of quality midwifery care - i.e. care delivered by midwives who are educated, licensed and regulated according to international standards and who are positively supported and respected in their role - is associated with positive health outcomes, increased maternal satisfaction, the reduction of maternal and neonatal mortality and morbidity and the efficient use of global resources [6-13]. In spite of strong evidence for the value of midwife-led continuity of care, there has been significant delay in its global implementation. This delay poses urgent and important questions, not only about the commitment of world leaders to the SDG goals, but also about the availability of the knowledge necessary for successful implementation of this type of care ${ }^{[14,15]}$

The birth of a baby is more than the birth of a child, it is the birth of a community that includes a mother, a father, siblings, grandparents, and others who are connected to that new life in networks of family and friends. Midwives stand with women in these moments of community creation, but they do more than just attend birth. Their work includes a spectrum of normal life events from sexuality education - contraceptive and abortion services through to antenatal, intrapartum and postnatal care including primary and acute health care. The holistic life-course approach of midwifery is based on a psychosocial, salutogenic model of care. The salutogenic approach of midwifery stands in contrast to a pathogenic model of care focused on disease and medical intervention ${ }^{[16]}$. This difference in approach is one of several barriers to the widespread implementation of midwifery ${ }^{[15,17]}$. The cultural and structural dominance of the pathogenic approach to health care diminishes the social and organisational position of midwives in society, limiting their effectiveness. In addition, midwives, who are predominantly women, doing women's work for and with women, face the challenges posed by gender inequity ${ }^{[17]}$. According to a recent editorial in the Lancet, it is projected that, if current trends continue, gender equity will not be realized until well into the next century ${ }^{[18]}$. In the case of midwives, it is well known that their place in health care has been systematically eroded and marginalized by patriarchal systems ${ }^{[15]}$.

However, change appears to be coming. Women and midwives are uniting their strengths and are joined by influential global partners and donors who understand the importance of midwifery for the attainment of Sustainable Developments Goals 3 and $5^{[18]}$. Using the motto 'Nothing about us, without us', women are recognising the need to be an integral part of the necessary change to global patriarchal dominance ${ }^{[17,19]}$. This also includes a shift in the global paradigm from a biomedical model to a psychosocial model of maternity care, a move that will allow midwife-led care to realise its promising potential ${ }^{[11,20,21]}$. This will not happen, however, until midwives develop their ability to influence decisions made at all levels of society - including, among others, health organisations, academic institutions, government institutions, and financial institutions ${ }^{[22,23]}$.

The history of midwifery and its current place in health systems around the world have, and continue to, limit the influence of midwives in policy decisions regarding maternity care. Given this situation, how can we cultivate the professional growth and the agency needed for midwives to take on their vital role in making and implementing policy? Topdown approaches - mandates from ministries of health or programmes imported by nongovernmental agencies - have proven ineffective in strengthening the profession. Among "bottom-up" strategies, 'twinning' has been offered as a promising way to promote the confidence of midwives and thereby strengthen the profession of midwifery and establish its voice as essential to maternity care policy and provision ${ }^{[24]}$

What is twinning? We describe the process in more detail below, but in brief, twinning involves the reciprocal exchange of knowledge and skills. In theory, twinning collaborations help midwives to support other midwives in pushing for the changes that are so urgently needed ${ }^{[24]}$. However, there is a lack of systematic research into twinning, resulting in a paucity of knowledge about how twinning works and what factors hinder and facilitate its 
potential to effectively strengthen the agency of midwives $\left[{ }^{[13,25]}\right.$. Without evidence about the process and value of twinning, implementing twinning programmes risks becoming a waste of precious time and funding. A stronger evidence base is needed in order to understand whether twinning has its promised potential, and, if so, to develop a clearer understanding of what twinning is, how it works, and how to optimise its potential. In this thesis we address this knowledge gap.

We begin this introductory chapter by describing the historical context of twinning. We go on to track its development as a form of collaboration between associations of midwives globally and to differentiate twinning from other classical forms of aid. To get a better understanding of the context in which the twinning projects studied in this thesis were initiated and conducted, we offer background information on those projects. After presenting the four main research questions addressed in the thesis we conclude with a basic outline of the following five chapters.

\section{HISTORY OF TWINNING}

The ability to learn from each other and exchange tangible goods as well as knowledge and skills is a central part of human interaction [26]. These exchanges have taken place between individuals, groups, nations and cultures, and while the process may not have carried the name 'twinning', the core principles are similar ${ }^{[27]}$. The first mention of twinning in the literature, as a specific type of collaboration, was in 1945 when the twinning of towns from different countries was developed as a way of uniting countries with the aim of protecting the world from another war ${ }^{[28]}$. This sparked all kinds of twinning collaborations between different types of institutions and for different causes. There have been twinning projects between hospitals, schools, and even toilets [29-31]! Twinning as a term has positive connotations and is used as a buzz word for all kinds of collaborations ${ }^{[32]}$.

In 1976, a twinning project between midwives was described for the first time in the official journal of the Royal College of Midwives ${ }^{[33]}$. This collaboration was between midwives from the United Kingdom and Germany. Since then, there has been a steady growth in the number of collaborations between midwives globally, including twinning between Tanzania and Canada, Japan and Mongolia, Uganda and the UK, the UK and Nepal, Sierra Leone and the Netherlands, the Netherlands and Iceland, Mali and Switzerland, the Netherlands and Morocco, and Ghana and Sierra Leone ${ }^{[24,34-40]}$

These twinning collaborations were initiated for various reasons and were organised differently depending on specific needs and context; however, most were between two groups of midwives and many share the general aim of reciprocal learning. The size and composition of the groups reflect the goals and organisation as well as the financial capacity of each twinning collaboration. Around the turn of the century, the International Confederation of Midwives (ICM), an organisation that unites one million midwives in 142 associations of midwives globally, recognized twinning for its potential to strengthen the capacity of associations of midwives. In an effort to realise its vision - to increase the quality of midwifery care and to ensure that every women worldwide has access to a midwife the ICM incorporated twinning into the ICM overall strategy and developed a manual to encourage and support twinning between associations of midwives [24].

\section{TWINNING IS NOT AID}

Although an unambiguous definition of twinning is missing in the literature, a number of values and beliefs appear to be shared by twinning collaborations. The importance and intention of reciprocity lies at the core of twinning and is stressed in all available twinning guides [24, 28, 41-43]. This reciprocity is expressed through the exchange of gifts. These 'gifts' come in many shapes and forms and can include knowledge, skills, goods, trust, collegiality, funding, and volunteerism. This is essentially different from other forms of traditional aid where the (financially) powerful give to the (financially) powerless, keeping the poor in the grip of the rich [27, 44-46]. However, in practise, the habit of perceiving and implementing twinning in a similar way to other forms of aid is common and can be a real and substantial challenge to successful twinning. This habit is exacerbated by the fact that, in the case of midwifery, most twinning collaborations are between a midwife organisation from a low or middle income country (LMIC) and an organisation in a high income country $(\mathrm{HIC})$.

Twinning is about each midwife association discovering their own strengths and capacities, recognising their value and exchanging these, like gifts, for other strengths and capacities. As an example from our own experience, the twinning partnership between Sierra Leone and the Netherlands demonstrated that most midwives in Sierra Leone had the strong capacity to speak in public, a skill much less well developed amongst many Dutch midwives. This skill was shared with the Dutch group, and the Dutch group shared their capacity to access academic online libraries for evidence-based midwifery practise, a skill much less well developed amongst many Sierra Leone 'twins'. 


\section{SETTING THE SCENE FOR THE TWINNING COLLABORATIONS STUDIED FOR THIS THESIS}

Two twinning collaborations were studied for this thesis. One was a four-year (2013-2016) twinning collaboration between the Sierra Leone Midwives Association (SLMA) and the Royal Dutch Association for Midwives (KNOV). The other was a three-year (2014-2017) twinning collaboration between the Association of Moroccan Midwives (AMSF) and the Royal Dutch Association for Midwives (KNOV).

The Sierra Leone and Dutch midwives' twinning collaboration came about as a subproject of a co-operation between many partners including the Dutch and the Sierra Leone Governments, focusing on strengthening midwifery care in Sierra Leone. Although there was no clear evidence to support the intervention of twinning in 2014, the collaboration organisers felt that it might be a good way to address the overall aim. The full project was funded by the Dutch government, and the International Confederation of Midwives played a key role in guarding it, by supporting both associations to play an equal part [24].

One-to-one twin pairing was introduced in the second year of this collaboration as a way of trying to overcome issues of "ownership", where no pro-active responsibility was taken for the proposed activities. The one-to one twinning intervention brought with it new logistical and financial challenges as it entailed the organisation of extra exchange visits, conference attendance, and workshops for 25 pairs of midwives. This made it necessary to obtain additional finances. The KNOV responded with the organisation of crowd funding as well as creating a charitable organisation, midwives4mothers. At the same time, the Sierra Leone midwives started making products, such as baby and placenta demonstration dolls, that were sold at conferences to generate funds ${ }^{[47]}$. Both enterprises were highly successful. An independent final evaluation of the collaboration showed many positive results - expected and unexpected - of twinning, such as the introduction and operationalisation of two 4-wheel-drive ambulances in a rural area in Sierra Leone. It also called attention to the problems of twinning. In this case we learned that the core principles of reciprocal exchange were strained by the size of the group as well as the distance between the associations geographically, financially, and culturally ${ }^{[48]}$. These lessons learned were used to adapt the on-going project, the twinning guide, and future twinning collaborations.

The twinning collaboration between Dutch and Moroccan midwives benefited from what we learned in Sierra Leone. We decided, for example, that the financial, geographical, and cultural gaps between the associations needed to be smaller before the choice of twinning partner was made. The fact of a large Moroccan community in the Netherlands, which made it possible to include several Dutch midwives of Moroccan origin in a Netherlands-Morocco collaboration, allowed us to address some of those gaps. Language issues were approached by incorporating two multilingual Arabic/French/Dutch/English midwife translators as part of the project management team who attended all activities. The numbers of midwives paired as twins was reduced to 19 pairs and funding was organised through a combination of crowd funding, self-funding (by twins), support from the private sector, and the sale of 40.000 self-designed Moroccan baby hats as well as other products [47]. This made the project financially self-sustainable. The project was evaluated by means of a baseline, midterm and final evaluation that was done independently from the studies for this thesis.

\section{GROUP AND ONE-TO-ONE TWINNING}

One aspect that differentiates the twinning collaborations studied for this thesis and other twinning collaborations is that each midwife, as part of the group, was appointed a specific midwife 'twin' from the other group. This one-on-one twinning was developed in 2014 as a creative and organic way to attempt to overcome issues of 'ownership' within the Netherlands-Sierra Leone collaboration, as described above.

From the onset of the Sierra Leone and the Netherlands twinning collaboration, it was clear that the contexts of these two associations were substantially different. Sierra Leone had just come out of a civil war, leaving a disbanded midwife association that needed a complete revival, whilst the Dutch midwife association had existed for more than 100 years and was well-established, stable and thriving. The fragile post-war context of Sierra Leone caused the team from the Sierra Leone midwife association to be fractured both physically and emotionally. In turn, the team from the Dutch midwife association experienced difficulties in understanding and operating within this context. This caused paralysis in the collaboration that was hard to substantiate but involved issues of problem ownership for the teams of both organisations ${ }^{[36]}$. A year after the project commenced, these challenges called for a shift in the project's approach.

Creating a platform for individuals to share personal values and build relationships of trust had been shown to positively impact collaborations and increase personal commitment to, and ownership of, the aims of the overall project ${ }^{[49}$. Based on this premise, twenty individual volunteer midwives from each association were recruited and paired one-to-one, to work on tangible self-chosen sub-projects that matched the overall project aims. On the Sierra Leone side, these twins had been involved in the previous workshops as a group, without being appointed a specific role or twin. On the Dutch side there was involvement from a small project management team only, so twins needed to be recruited to match the Sierra Leone group. 


\section{A STEP BY STEP TWINNING GUIDE}

In both twinning projects studied for this thesis, individual twins were paired on the basis of their midwifery expertise; teachers with teachers, students with students, practising midwives with practising midwives, etc. Twin pairs were supported in their collaboration by a Dutch-Sierra Leone or Moroccan-Dutch project team following a ten-step guide. This guide was developed through a bottom up process, during the three-year Sierra LeoneDutch collaboration by the project team using input from evaluations and feedback from the twin pairs ${ }^{[48]}$. The ten-step guide includes forming a bi-lateral project team, setting joint goals accompanied by a budget, having regular workshops with attention for cross cultural collaboration and communication, organising exchange visits to each other's countries and regular celebration of achievements as well as the joint attendance at a midwives' conference and a final exhibition of the achievements in both countries (see Figure 1). This step-by-step guide was developed through a process of trial and error and was made available in a Web-Application in French and English ${ }^{[41,50]}$.

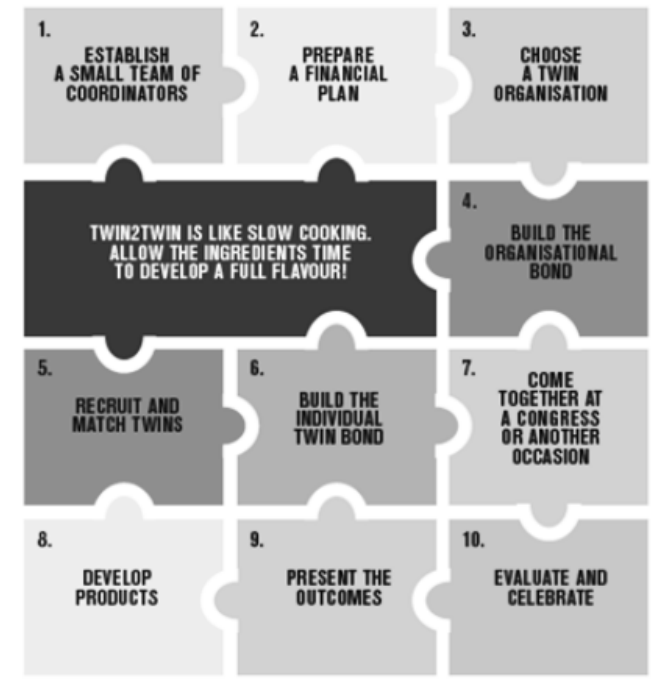

Figure 1. The ten steps- from twin2twin guide.

\section{AIM OF THIS THESIS}

At the start of the Sierra Leone and Dutch twinning project, twinning, and more specifically, one-to-one twinning, had no evidence base. A search for this evidence generated questions about how twinning was defined, what hindered and facilitated its success, and what role one-to-one twinning plays in the process. Talking with others who had a similar curiosity and passion for the subject of midwives and twinning resulted in a serious exploration of the subject by a multi-disciplinary research team, the results of which are presented in this thesis.

In this thesis, we explore how twinning collaborations work and what factors enhance or hinder its potential to strengthen the agency of midwives. The following research questions are addressed:

1. How can the concept of twinning in healthcare be defined? (chapter 2)

2. What factors are critical for the success of twinning in healthcare? (chapter 3 )

3. How did midwives from Morocco and the Netherlands experience twinning and what influenced their professional growth? (chapter 4)

4. What is the impact of the personal, one-to-one twin relationship for twinning? (chapter 5)

\section{OUTLINE OF THIS THESIS}

1. Chapter 2 addresses the lack of clarity about the concept 'twinning' by means of a concept analysis.

2. Chapter 3 presents the results of a Delphi process - done amongst midwife experts in twinning - seeking consensus on the factors that are critical for successful twinning.

3. Chapter 4 explores the effects of twinning on midwives participating in the MoroccanDutch twinning project, with specific reference to cultural diversity.

4. Chapter 5 describes the impact of one-to-one twin pairs in two twinning collaborations between midwives from Morocco and the Netherlands and Sierra Leone and the Netherlands.

5. Chapter 6 is a reflection on our findings as seen through the lens of complexity theory. 


\section{REFERENCES}

1. United Nations (UN) Sustainable Development Goals. 2015 [cited 2020 January 10]; Available from: https:// www.who.int/sdg/targets/en/.

2. United Nations (UN) Sustainable Development Goals. SDG 3. 2015 [cited 2020; Available from: https://www. who.int/sdg/targets/en/.

3. United Nations (UN) Sustainable Development Goals. SDG 5. 2015 [cited 2020] Available from: https:// sustainabledevelopment.un.org/sdg5.

4. World Health Organization (WHO). Universal Health Coverage fact sheet.2020 [cited 2020] Available from: https://www.who.int/news-room/fact-sheets/detail/universal-health-coverage-(uhc).

5. White Ribbon Alliance. What Women Want. 2019 [cited 2020] Available from: https://static1.squarespace.com/ static/5aa813dd3917ee6dd2a0e09e/t/5d1120ccdf7cbc0001b99c57/1561403606693/What-Women-Want_ Global-Findings.pdf P45.

6. Van Lerberghe, W., Matthews, Z., Achadi, E., Ancona, C., Campbell, J., Channon, A.,... \& Koblinsky, M. (2014). Country experience with strengthening of health systems and deployment of midwives in countries with high maternal mortality. The Lancet, 384(9949), 1215-1225. http://dx.doi.org/10.1016/S0140-6736(14)60919-3.

7. Lawn, J. E., Blencowe, H., Oza, S., You, D., Lee, A. C., Waiswa, P.,... \& Mathers, C. (2014). Every Newborn: progress, priorities, and potential beyond survival. The Lancet, 384(9938), 189-205. http://dx.doi.org/10.1016/ S0140-6736(14)60496-7.

8. ten Hoope-Bender, P., de Bernis, L., Campbell, J., Downe, S., Fauveau, V., Fogstad, H.,... \& Renfrew, M. J. (2014). Improvement of maternal and newborn health through midwifery. The Lancet, 384(9949), 1226-1235. http://dx.doi.org/10.1016/S0140-6736(14)60930-2

9. Renfrew, M. J., McFadden, A., Bastos, M. H., Campbell, J., Channon, A. A., Cheung, N. F.,... \& McCormick, F. (2014). Midwifery and quality care: findings from a new evidence-informed framework for maternal and newborn care. The Lancet, 384(9948), 1129-1145. http://dx.doi.org/10.1016/ S0140-6736(14)60789-3.

10. Renfrew, M. J., Homer, C., Downe, S., McFadden, A., Muir, N., Prentice, T., \& ten Hoope-Bender, P. (2014). Midwifery: an executive summary for the Lancet's series. The Lancet, 384(1), 8.

11. Homer, C. S., Friberg, I. K., Dias, M. A. B., ten Hoope-Bender, P., Sandall, J., Speciale, A. M., \& Bartlett, L. A. (2014). The projected effect of scaling up midwifery. The Lancet, 384(9948), 1146-1157. http:// dx.doi. org/10.1016/S0140-6736(14)60790-X.

12. Mwaniki, M. K. (2016). Quality in provision of maternity services: the missing link in health-care investments in LMICs? The Lancet Global Health, 4(11), e769-e770. http://dx.doi.org/10.1016/S2214-109X(16)30239-X.

13. United Nations Population Fund (UNFPA). (2014) The State of the World's Midwifery, a universal pathway. A woman's right to health. Editor Bernis L.D. UNFPA, ICM, WHO: New York. ISBN: 978-0-89714-026-3 Available from: http://www.unfpa.org/sowmy.

14. Sandall, J., Soltani, H., Gates, S., Shennan, A., \& Devane, D. (2016). Midwife-led continuity models versus other models of care for childbearing women. Cochrane database of systematic reviews, (4). https://doi. org/10.1002/14651858.CD004667.pub5.
15. Renfrew, M. J., Ateva, E., Dennis-Antwi, J. A., Davis, D., Dixon, L., Johnson, P.,... \& McFadden, A. (2019). Midwifery is a vital solution-What is holding back global progress?. Birth (Berkeley, Calif.), 46(3), 396. doi: 10.1111/birt.12442.

16. Bryers, H. M., \& Van Teijlingen, E. (2010). Risk, theory, social and medical models: a critical analysis of the concept of risk in maternity care. Midwifery, 26(5), 488-496. https://doi.org/10.1016/.j.midw.2010.07.003.

17. 17. Coe, l. (2019). Feminism is for everybody. The Lancet, 393(10171), 493. https://doi.org/10.1016/S0140$6736(19) 30239-9$.

18. The Lancet. (2020). 2020: a critical year for women, gender equity, and health. PMIS: 31908270 . Editorial, Jan 4;395(10217):1. doi: 10.1016/S0140-6736(19)33170-8.

19. Walsh, D. M. Christianson, and Stewart M. (2015). Why midwives should be feminists. MIDIRS Midwifery Digest, 2015. 25(2): p. 154-160. MIDIRS Midwifery Digest. 25(2):154-160.

20. Van Teijlingen, E. (2017). The medical and social model of childbirth. Kontakt, 19(2), e73-e74. doi: 10.1016/j. kontakt.2017.03.001.

21. Sandall, J., Devane, D., Soltani, H., Hatem, M., \& Gates, S. (2010). Improving quality and safety in maternity care: the contribution of midwife-led care. The Journal of Midwifery \& Women's Health, 55(3), 255-261. https:// doi.org/10.1016/.jmwh.2010.02.002

22. Gill K., Pande R., Malhotra A. (2007). Women deliver for development. The Lancet, 2007. 370(9595): p. 13471357. https://doi.org/10.1016/S0140-6736(07)61577-3.

23. Mosedale, S. (2014). Women's empowerment as a development goal: taking a feminist standpoint. Journal of International Development, 26(8), 1115-1125. https://doi.org/10.1002/jid.3050.

24. International Confederation of Midwives (ICM) twinning manual. (2014). Editor Moyo N. T. the Hague.

25. United Nations Population Fund (UNFPA). (2019). Sexual and Reproductive Health and Rights: An Essential Element of Universal Health Coverage. Editor: UNFPA. New York.

26. Mauss, M., \& Halls, W. D. (1954). The gift: Forms and functions of exchange in archaic societies (№. 378).

27. Kowalski, R. (2011). The Gift-Marcel Mauss and international aid. Journal of Comparative Social Welfare, 27(3), 189-205. https://doi.org/10.1080/17486831.2011.595069.

28. World Health Organization (WHO). (2001). Guidelines for city twinning. WHO Regional office Europe. [cited 2020]. Available from: https://apps.who.int/iris/bitstream/handle/10665/107370//E74547.pdf?sequence=1.

29. Jiang, H., Erickson, J. I., Ditomassi, M., \& Adams, J. M. (2012). Promoting a culture of international professional practice for nursing through a twinning relationship. JONA: The Journal of Nursing Administration, 42(2), 117122. doi: 10.1097 /NNA.0b013e318243384e.

30. Qaddoumi, I., \& Bouffet, E. (2009). Supplementation of a successful pediatric neuro-oncology telemedicinebased twinning program by e-mails. Telemedicine and e-Health, 15(10), 975-982. https://doi.org/10.1089/ tmj.2009.0043

31. Cord. Toilet twinning.org (2009). [cited 2020]. Available from: http://www.toilet twinning.org/twin-your-toiletnow/

32. Cornwall, A., \& Brock, K. (2005). What do buzzwords do for development policy? A critical look at participation', 'empowerment' and 'poverty reduction'. Third world quarterly, 26(7), 1043-1060. https://doi. 
org/10.1080/01436590500235603.

33. Christian, K. "Twinning". (1978) Midwives chronicle 91 (1082): p. 59

34. midwives4mothers charity. South - South Twinning. [cited 2019]. Available from: https://midwives4mothers.nl/ projecten/twinning-south-to-south/

35. midwives4mothers charity. Twinning up North. Twinning project Iceland and the Netherlands. [cited 2019]. Available from: https://midwives4mothers.n//projecten/twinning-up-north/.

36. Cadée, F., Perdok, H., Sam, B., de Geus, M., \& Kweekel, L. (2013). 'Twin2twin'an innovative method of empowering midwives to strengthen their professional midwifery organisations. Midwifery, 29(10), 1145-1150. https://doi.org/10.1016/j.midw.2013.07.002.

37. Kemp, J., Bannon, E., Mwanja, M., Tebuseeke, D.(2018). Developing a national standard for midwifery mentorship in Uganda, International Journal of Health Governance, 23(1): p.81-94. https://doi.org/10.1108/ IJHG-09-2017-0051.

38. Ireland, J., Van Teijlingen, E., \& Kemp, J. (2015). Twinning in Nepal: the Royal College of Midwives UK and the Midwifery Society of Nepal working in partnership. Journal of Asian Midwives, 2(1), 26-33. http://ecommons. aku.edu/jam/vol2/iss $1 / 5 /$.

39. Japanese Midwives Association (2018) Midwives Reducing Obesity in Mongolia. Midwives For Life. [cited 2018] Available from: http://www.m2025-weobservatory.org/midwives-reducing-obesity-in-mongolia.htm.

40. Sandwell, R., Bonser, D., Hebert, E., Kilroy, K., Leshabari, S., Mwanga, F., ... \& Moritz, A. (2018). Stronger together: midwifery twinning between Tanzania and Canada. Globalization and health, 14(1), 1-10. doi: 10.1186/ s12992-018-0442-x.

41. Geus de M., Kweekel L. (2012) Royal Dutch Organisation of Midwives (KNOV), twin2twin midwives empower midwives. Editors Cadée F., Perdok H. 2012, Amsterdam. ISBN: 9789081051057.

42. Breiddal, S. (2009). The How-To Guide to Hospice Palliative Care Twinning Projects Health Canada. Available from: https://www.canada.ca/en/health-canada/services/health-care-system/reports-publications/palliativecare/guide-hospice-palliative-care-twinning-projects-2009.html.

43. Jones, M. L., \& Blunt, P. (1999). 'Twinning'as a method of sustainable institutional capacity building. Public Administration and Development, 19(4), 381-402. https://doi.org/10.1002/(SICI)1099-162X(199910)19.

44. Moyo, D. (2009). Dead aid: Why aid is not working and how there is a better way for Africa. Macmillan. ISBN 978-0-141-03118-7.

45. Chang, H. J. (2010). Bad Samaritans: The myth of free trade and the secret history of capitalism. Bloomsbury Publishing USA. ISBN10 1596915986.

46. Mawdsley, E. (2012). The changing geographies of foreign aid and development cooperation: contributions from gift theory. Transactions of the Institute of British geographers, 37(2), 256-272. https://doi.org/10.1111/j.14755661.2011.00467.x.

47. midwives4mothers charity.(2020). [cited 2020]. Available from: https://midwives4mothers.n//en/home-2/.

48. Herschderfer, K., Perdok, H. (2012) Assessment of the KNOV-SLMA Twin to Twin Programme Perceptions and experiences of twinned midwives and lessons learned. (unpublished report) Royal Tropical Institute, KIT: Amsterdam
49. Vangen, S., \& Huxham, C. (2003). Nurturing collaborativerelations: Building trustininterorganizational collaboration. The Journal of Applied Behavioral Science, 39(1), 5-31. https://doi.org/10.1177/0021886303039001001

50. twintowin.(2017). Web Application. [cited 2020] Available from: https:/apps.apple.com/us/app/twintowin/ id1247269680. 


\section{ABSTRACT}

\section{BACKGROUND}

Inequities in health have garnered international attention and are now addressed in Sustainable Development Goal 3 (SDG3), which seeks to 'promote well-being for all'. To attain this goal globally requires innovative approaches, one of which is twinning. According to the International Confederation of Midwives, twinning focusses on empowering professionals, who can subsequently be change-agents for their communities. However, twinning in healthcare is relatively new and because the definition and understanding of twinning lacks clarity, rigorous monitoring and evaluation are rare. A clear definition of twinning is essential for the development of a scientific base for this promising form of collaboration.

\section{METHOD}

We conducted a Concept Analysis (CA) of twinning in healthcare using Morse's method. A qualitative study of the broad literature was performed, including scientific papers, manuals, project reports, and websites. We identified relevant papers through a systematic search using scientific databases, backtracking of references, and experts in the field.

\section{RESULTS}

We found nineteen papers on twinning in healthcare. This included twelve peer reviewed research papers, four manuals on twinning, two project reports, and one website. Seven of these papers offered no definition of twinning. In the other twelve papers definitions varied. Our $\mathrm{CA}$ of the literature resulted in four main attributes of twinning in healthcare. First, and most frequently mentioned, was reciprocity. The other three attributes were that twinning: 2) entails the building of personal relationships, 3) is a dynamic process, 4) is between two named organisations across different cultures. The literature also indicated that these four attributes, and especially reciprocity, can have an empowering effect on healthcare professionals.

\section{CONCLUSIONS AND IMPLICATIONS FOR PRACTISE}

Based on these four attributes we developed the following operational definition: Twinning is a cross-cultural, reciprocal process where two groups of people work together to achieve joint goals. A greater understanding and a mature definition of twinning results in clear expectations for participants and thus more effective twinning. This can be the starting point for new collaborations and for further international studies on the effect of twinning in healthcare.

\section{KEY WORDS}

Healthcare professionals, international collaboration, midwives, nurses, physicians, reciprocity, twinning.

\section{BACKGROUND}

Can twinning in healthcare play a role in sustainable development globally? At the United Nations General Assembly 2015 (UNGA) the 193 leaders adopted seventeen Sustainable Development Goals (SDGs) [1]. SDG 3 states that by 2030 we will 'Ensure healthy lives and promote well-being for all at all ages.' In an era where the gap between rich and poor within and between societies is ever widening ${ }^{[2,3]}$, attaining SDG 3 will be a challenging task demanding a variety of collaborative approaches as stated in SDG17 ${ }^{[4]}$. Twinning is one of these approaches, a method that brings together health professionals across cultures in an effort to improve healthcare by empowering these professionals ${ }^{[5]}$. And indeed twinning does appear to have great potential for this purpose. Reports from the Swedish International Development Co-operation Agency (SIDA) describe twinning to have 'potential advantages over other forms of development co-operation as it encourages organisational learning and sustainable development' ${ }^{[6]}$. According to SIDA the potential of twinning has not yet been fully exploited. At present twinning in healthcare is implemented in a variety of ways ranging from straight forward linking of institutions to organising exchange visits to complex processes of bilateral development between healthcare professionals. This variation makes it difficult to pin down the essential features of twinning.

Historically twinning can be traced back to town twinning that was encouraged by the European Community for many decades following the Second World War. Twinning was defined as 'a formal and substantive collaboration between two organisations and/or cities' [7,8]. It was intended as a way to improve international relations and reconciliation by fostering friendship and increasing understanding between different cultures and former foes ${ }^{\left[{ }^{[9]}\right.}$. Town twinning caught on and was soon joined by twinning of various organisations in different settings from different countries. At the start, these activities ran in conjunction to Town Twinning but they soon functioned independently to the point that we now have twinning going on all over the globe in many different shapes and contexts, with examples ranging from twinning between diabetes associations of Mozambique and the UK to improve diabetes care [10], the Tonga twinning Programme between hospital staff of Australia and Tonga ${ }^{[1]]}$ to "toilet twinning" between any two toilets worldwide to improve world sanitation [12].

If twinning is to play an important role globally in empowering healthcare professionals to perform to their potential ${ }^{[7,13]}$, further scientific studies need to be done to underpin assumptions of effectiveness. Without definitional clarity and a full understanding of the concept, it is difficult to assess the value of twinning or to discover which aspects of twinning contribute to its success. In this paper we offer a concept analysis (CA) of twinning in healthcare using a critical exploration of the literature where 'the literature is used as data' ${ }^{[4]}$, aiming for an operational 


\section{METHOD}

Given the lack of definitional clarity, we performed a CA to gain a clearer view on the definition and key attributes of twinning as a type of international collaboration. Table 1. gives an explanation of the CA terminology used in this article.

\section{Table 1. Concept Analysis terminology.}

\begin{tabular}{ll} 
Concept Analysis terminology \\
\hline Concept & Conceptual representations of a phenomenon \\
Attribute & A characteristic of a concept that appears over \& over again \\
Boundary & What is \& what is it not part of the concept (delimiting cases) \\
Variation & A similar term with some overlapping attributes \\
Consequence & The effect brought about by attributes of a concept \\
\hline
\end{tabular}

\section{MORSE'S METHOD OF CONCEPT ANALYSIS}

We chose Morse's method ${ }^{[14]}$ for our CA because it offers a useful framework whereby the pragmatic utility of a concept can be established taking into account the dynamic context in which the concept is used. According to Morse, concepts can either be emerging or mature. A mature concept is well defined, has clearly described attributes, delineated boundaries and documented preconditions and outcomes. The degree of maturity determines the usefulness of the concept ${ }^{[14]}$. In order to use concepts in research, they need to be mature. For this reason it is necessary to evaluate the level of maturity of a concept ${ }^{[15]}$.

\section{APPLYING MORSE METHOD TO TWINNING IN HEALTHCARE}

Figure 1 describes the steps we use to conduct our concept analysis.

\begin{tabular}{l}
\hline Literature search on twinning in healthcare using extraction document \\
Analytical question that determines the process of analysis formulated \\
Profound understanding of the material \\
Matrix with analytical questions \& answers "Yes," "No" or "Exceptions" drawn up \\
Results compiled \\
Pragmatic utility established
\end{tabular}

Figure 1. Process of Concept Analysis according to Morse.

Between December 2014 and August 2015 we did a literature search using Ebscohost
(Psycinfo, Cinahl), Embase, Medline, Pubmed and Web of Science (medical/social sciences). This search and selection was done by the first author in close collaboration with the second author. The key search terms were 'healthcare professionals', 'twinning' and 'international' (Table 2). The search was limited to papers published from 1985 - when digitalisation of Medline began - to 2015, with no language restrictions. All papers that mentioned the term twinning and healthcare professionals in the title and/or the abstract were selected. The full articles were then obtained and read and any that did not deal with twinning as a form of collaboration were discarded. To extend our search, we hand searched the reference lists of included articles and consulted experts in the field. As all literature is seen as data in a $\mathrm{CA}$, a rich sample including discussion papers, final project reports and manuals pertinent to twinning were also included.

Table 2. Search strategy for $C A$ on twinning.

\section{Search Strategy}

Midwi* OR Nurse* OR Health personnel OR

Healthcare workers OR Doctor* OR Physician*

International OR Intercultural OR Cultural

Subsequently, we used an extraction document to collect the main characteristics for each paper, such as dates, definitions, assumptions, boundaries, contexts (such as country), variations, attributes, type of healthcare professionals, source of funding and quotations to facilitate the discussion. The maturity of the concept twinning in healthcare was assessed using the indices: definition, attributes, boundaries, preconditions and consequences [15]. As the understanding of the data deepened analytical questions were formulated and reformulated several times. The three final analytical questions used to distil attributes and boundaries from the data were:

1. What attributes make twinning distinctly different from other forms of collaboration?

2. Is reciprocity a core value of twinning?

3. Are the other emerging attributes and boundaries present in all papers?

The extraction document was used to tabulate the answers to question 1. These data were then rigorously analysed and incorporated in a matrix shown in Table 3. This matrix allowed oversight and critical analyses of the literature helping to clarify the concept and develop an operational definition by distilling the attributes, boundaries and consequences from the data. We then used the matrix to answer questions 2. and 3. with 'Yes', 'No' or 'Exception' when the answer was unclear. The data extraction and analysis was primarily done by the first author but was scrutinised by all authors. 

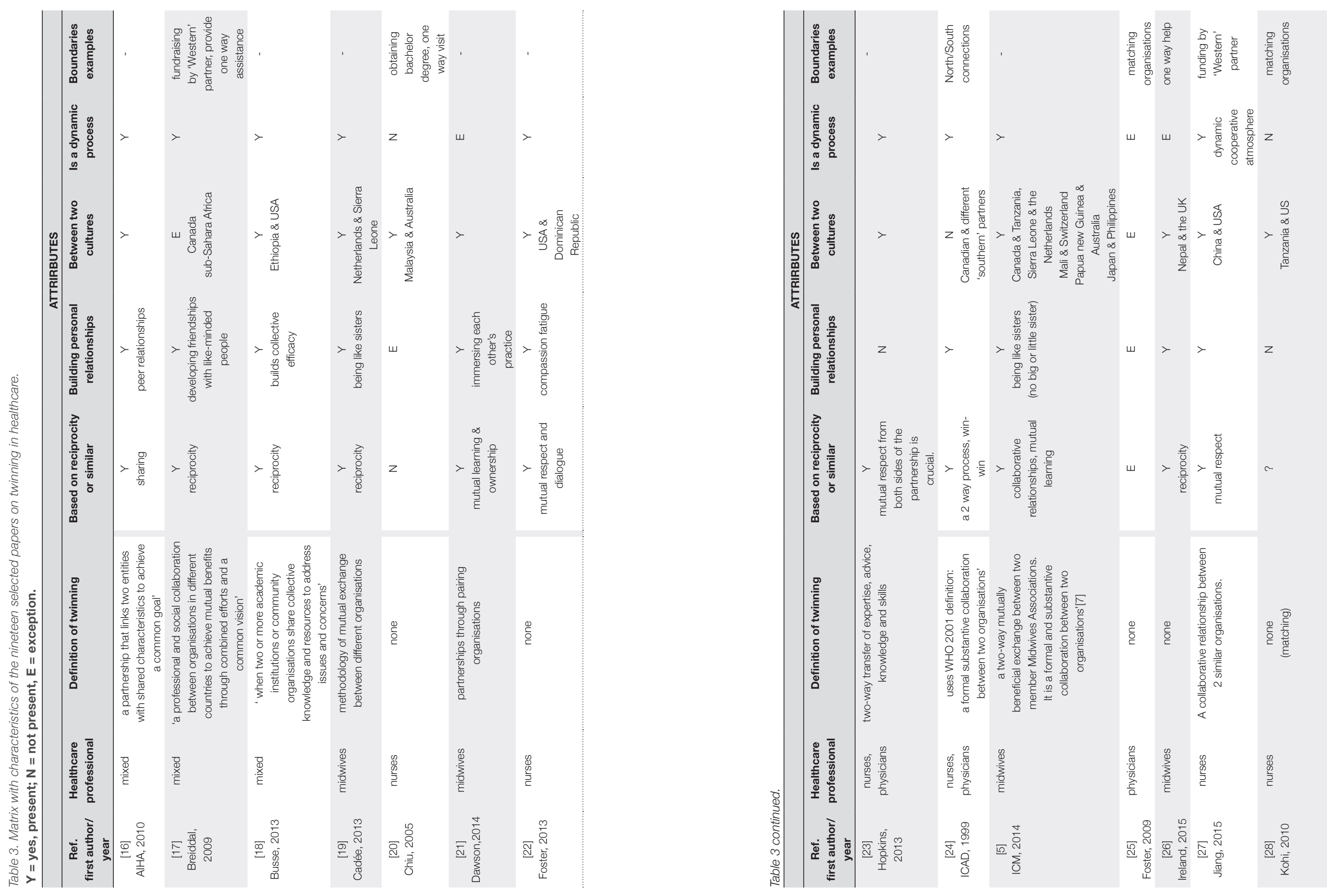


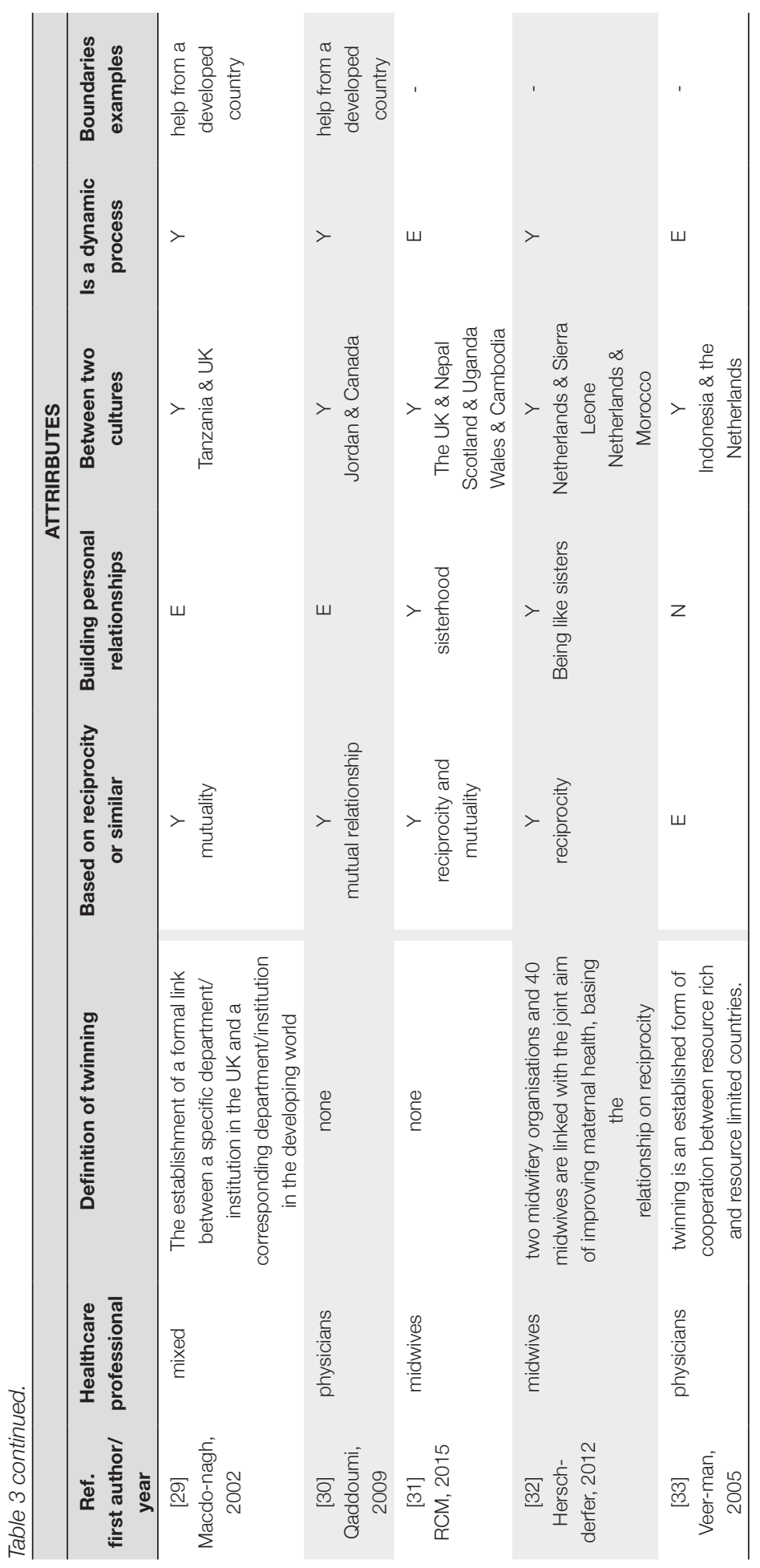

\section{RESULTS}

Of the 359 hits in the search, 341 remained after excluding double papers. We excluded a further 311 papers based on the title and the abstract, as they did not meet our inclusion criteria. Many of the excluded papers were about congenital twins. Subsequently, after fully reading the remaining 30 papers, 12 papers were selected which met all the criteria. We found an additional 7 papers by hand searching the reference list of selected papers and by consulting two experts on twinning in healthcare. These were manuals and reports that were not available in the scientific databases but relevant for a concept analysis of the broad literature, leaving us with 19 articles in total as shown in Figure 2.

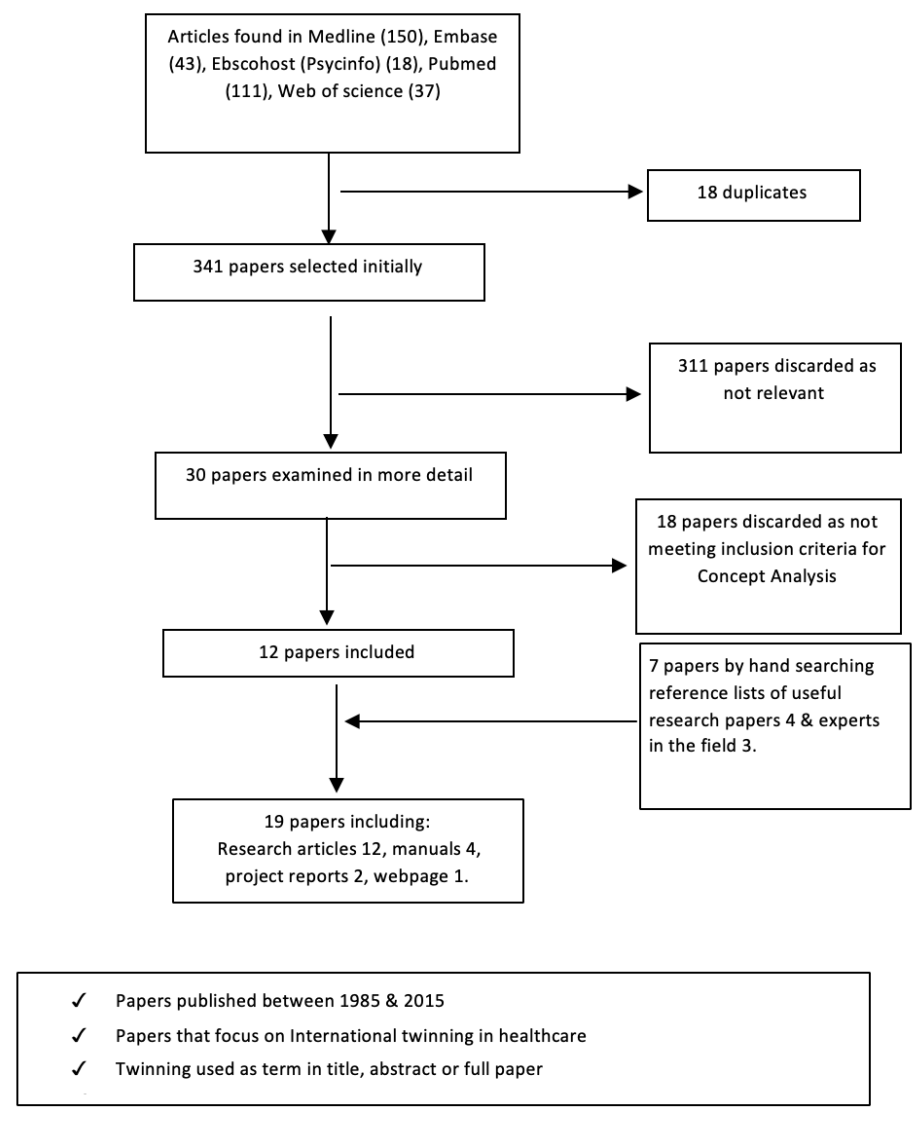

Figure 2. Literature review process for CA on twinning in healthcare.

Twelve papers were peer reviewed research articles and two were final project reports of twinning projects. The four manuals and one web page were written to guide different 
healthcare professionals in twinning collaborations. Using the indices on the maturity of the concept twinning according to Morse, the data showed twinning to be an emerging concept with competing definitions, lacking clarity and without clearly identified attributes, boundaries and consequences.

\section{DEFINITIONS OF TWINNING IN HEALTHCARE}

Seven of the nineteen papers had no definition of twinning. Two of these seven papers used the term twinning as an adjective meaning 'matched' ${ }^{[16,17]}$. In the remaining twelve papers twinning was used as a verb indicating a process. Two papers used the definition from the WHO City Twinning Guide []]: 'twinning is a formal substantive collaboration between two organisations' ${ }^{[5,18]}$. Even though this definition originates from organisations of international standing, the definition has not been integrated into the broader literature or research on twinning in healthcare. The definitions in the other nine papers varied and lacked clarity. All definitions incorporated some type of collaboration by using terms such as partnership, collaboration, exchange, sharing, pairing, cooperation, linking or exchange.

\section{PARTICIPANTS IN TWINNING}

Of the nineteen papers, six described twinning projects in mixed (care and cure) healthcare teams where the nurses were generally included in a later phase of the project and the implementation of the project as well as reporting was in the hands of physicians ${ }^{[18-22]}$. Six papers describe twinning between midwives ${ }^{[5,23-27]}$, four between nurses ${ }^{[17,28-30]}$ and two between physicians. In the data on twinning projects with nurses and midwives the four emerging attributes as described below appeared more frequently than in the data with physicians where delimiting cases appeared more frequently demonstrating the boundaries of twinning.

\section{ATTRIBUTES OF TWINNING IN HEALTHCARE}

Four distinctive attributes of twinning appeared over and over, clarifying our understanding of the concept. The four attributes we identified are that twinning:

1. Is based on reciprocity,

2. entails the building of personal relationships,

3. is a dynamic process,

4. is between two named organisations across different cultures.

As twinning can only be defined by the distinguishing attributes, we did not include other commonly mentioned attributes such as learning, lasting for a substantial period of time, having a formal contract, being culturally sensitive, being (internally or externally) funded, developing leadership skills, exchanging ideas, knowledge or technology are not included as they are characteristic for many forms of collaboration and therefore not specific for twinning ${ }^{[1]]}$.

1. Twinning is based on reciprocity: The term reciprocity used in this paper is defined in the online Lexico Oxford dictionary as 'exchanging things with others for mutual benefit' ${ }^{[32]}$. Fourteen papers mention reciprocity as a core value of twinning suggesting it to be a defining feature of twinning programmes. Reciprocity is either used explicitly or implicitly with terms like "mutual", "2-way" or "sharing" (see Table 3). Characteristic quotes of reciprocity as a core value of twinning are:

'In situations where economic disparity exists, a conscious effort needs to be made to ensure that the Twinning relationship and activities are approached as equal partners.' $\mathrm{p} 10^{[5]}$

‘...building a twinning partnership is grounded in shared guiding principles that emphasize the importance of building long-term relationships...based on equality, reciprocity, shared responsibility.'p1312 [21]

Specifically, the dynamic cooperative atmosphere fosters learning through the sharing of respective experiences' p118 ${ }^{[30]}$

'The most successful twinning projects are those that are two-way - i.e. each organisation learns from the other. Two-way twinning is empowering to both organisations.' p25 [18]

2. Twinning entails the building of personal relationships: Twelve papers speak of the importance of building a personal relationship between twins. For example, twins in twinning projects between midwives are described as 'being like sisters', nurses and physicians talk of 'developing friendships with like-minded people' or 'being immersed in each other'. Additional quotes exemplify the importance of personal relationship:

'You must be open to examining your own attitudes and behaviour. Reflect honestly on your willingness to wait and watch (when appropriate), to learn from your twinning partner, to refrain from "fixing" and giving advice, and to become aware of assumptions or prejudgments.' p5 ${ }^{200}$

'Despite the language challenges and 7,300 miles that separate our 2 facilities, nurses speak a common language.' p121 [30] 
3. Twinning is a dynamic process: A similar pattern emerges in the literature with references to putting the accent on the twinning process with room for spontaneous development. Eleven papers mention this, using terms such as: 'flexibility', 'dynamic cooperative atmosphere', 'synergy' or 'collective efficacy'.

\section{Twinning is between two named organisations across different cultures: All} twinning projects in the selected papers occurred between two similar organisations, such as two hospitals ${ }^{[21]}$, two hospices ${ }^{[20]}$ two training facilities ${ }^{[29]}$ or two associations of health professionals ${ }^{[23,25]}$. Five of these papers specifically mention people to people twinning within these organisations ${ }^{[21,23,26,30]}$. Six definitions specifically mention that twinning takes place between two organisations. Three papers ${ }^{[20,22,33]}$ mention that twinning takes place between organisations from two different countries. In all other papers it is assumed that the relationship is between two named organisations in two different countries from, for example, Ethiopia and USA ${ }^{[21]}$, the UK and Nepal ${ }^{[2]}$ or Jordan and Canada ${ }^{[34]}$. From the data it becomes clear it is not so much the national border, but the difference in culture that is a requisite for twinning.

\section{BOUNDARIES OF TWINNING IN HEALTHCARE}

Several papers use the concept twinning but don't include the above attributes. These cases give us a clear view of the boundaries of twinning. This can be demonstrated by two of the papers which use the concept twinning as an adjective to mean 'matched' [16, 17], one paper that describes a case study of twelve Malaysian students to investigate why they want to do their studies abroad ${ }^{[28]}$ and one paper specifically mentions a one way, rich to poor, process 'it gives young paediatricians from the less affluent nations a chance to improve their skills' p106 ${ }^{[33]}$. In these last two papers the term twinning appears to be misplaced as it is one sided development and lacks the key attributes we describe above.

Of all nineteen papers, three describe twinning between the physicians, six in mixed teams and ten between the nurses and/or midwives (Table 3). The attribute of building a relationship is mentioned less frequently by those papers that discuss twinning between physicians ${ }^{[16,33}$ or mixed teams ${ }^{[22,34]}$. Building a personal relationship is less clear or absent when twinning is interpreted as 'matching' ${ }^{[16,17]}$ or as a strategy for obtaining a Bachelor's degree abroad [28]. Quotes that illustrate this boundary are:

'The demand for a degree for Malaysian nurses provided the impetus for this offshore post-registration nursing programme, developed as a twinning venture between an Australian university and a Malaysian private institution.' $046{ }^{[28}$
'The goal was to ensure that nursing students would have entry-level knowledge and skills in the care and treatment of people living with HIV.' p93 [17]

\section{CONSEQUENCES OF TWINNING IN HEALTHCARE}

In none of the nineteen papers used in this CA was the impact, or the consequences, of twinning systematically investigated by the authors. Our examination of the literature, however, uncovered a probable correlation between reciprocity and the empowerment of healthcare professionals. Empowerment is taken to mean 'a powerful catalyst for positive change' ${ }^{[35,36]}$. Thirteen authors make a point of emphasising the importance of reciprocity to achieve empowerment. Authors specifically mentioned this at least once under either a special heading ${ }^{[5,18,23,24,26,27]}$, or in the introduction ${ }^{[20,29]}$, the conclusion ${ }^{[21,22,25]}$ or abstract $[19,30]$. The following quotes illustrate the emphasis that authors put on reciprocity for empowerment.

Specifically, the dynamic cooperative atmosphere fosters learning through the sharing of respective experiences....twinning requires the implementation of shared strategies and developmental goals.' p118 ${ }^{130}$

'Recognising that the UK has much to learn from low-income countries, the meaning and process of true exchange, reciprocity and mutuality should be further explored.' p5 ${ }^{[27]}$

'Reciprocity is an important aspect of twinning as the RCM also hopes to strengthen midwifery in the UK.' p26

In the remaining five papers empowerment is not mentioned. In these papers twinning is seen as matching ${ }^{[16,17]}$ or the emphasis is on a specific end result such as obtaining a bachelor degree ${ }^{[28]}$ or the training of healthcare professionals ${ }^{[3,34]}$

\section{DISCUSSION}

\section{DEFINING TWINNING IN HEALTHCARE}

The literature on twinning in healthcare to date has been as diverse as its definitions. Analysing the literature using Morse's CA methodology led to the identification of four attributes that distinguish twinning from other types of international collaboration. These are that twinning 1) is based on reciprocity, 2) entails the building of personal relationships, 3) is a dynamic process, 4) is between two named organisations across different cultures. The 
data indicate that these four attributes, but foremost reciprocity, relate to empowerment. Empowerment can therefore be seen as a consequence of twinning. If twinning in healthcare continues without the use of a clear definition, enthusiasm about its potential will remain mere enthusiasm and the outcomes will be no more than the outcome described in one of the first papers written on twinning in healthcare 'we all learned a great deal' ${ }^{[37]}$. As a result of this CA the following definition can be formulated using the emerged attributes: Twinning is a cross-cultural reciprocal process where two groups of people work together to achieve joint goals.

\section{THE MATURITY OF THE CONCEPT TWINNING}

The concept twinning in the literature to date is broad and used as an everyday concept similar to its use in common language, its function being to facilitate communication of an abstract activity in daily life. Why was the concept twinning used in this way? We think that twinning as a concept has certain connotations that makes it appealing to use. After all, the innate congenital meaning of two separate beings that are born at the same time stirs the imagination. This makes twinning very visual and gives marketing value to the concept. This positive reputation of twinning may be the reason why the term to date has been used so freely.

In conducting this CA we have deepened our understanding of twinning and contributed to the maturity of concept (Morse). The analysis of the data has brought a clear consensual definition, with clearly described attributes and boundaries. Empowerment has emerged as a consequence (effect) of twinning. Other potential outcomes however cannot yet be fully described or demonstrated until the definition is integrated in the field and the outcomes are monitored.

\section{RECIPROCITY IN TWINNING IN LIGHT OF THE GIFT THEORY}

The first three attributes of twinning, that it 1) is based on reciprocity, 2) entails the building of personal relationships, 3) is a dynamic process, together with their interconnectivity can be better understood using the classic work of Mauss on 'the gift' ${ }^{[38]}$. While his work describes ancient societies, it remains relevant in 2016 and clearly demonstrates why reciprocity is an essential feature of twinning.

The importance of reciprocity for successful twinning emerged clearly from the data, yet most authors reported difficulty in bringing theory into practise because of problems with checks and balances. In Mauss's Gift theory three aspects to gift giving are described: that of the giver, the receiver, and the reciprocator, which assumes social interaction. This interaction is reflected in the attributes two and three of twinning. According to Mauss 'The unreciprocated gift still makes the person who has accepted it inferior, particularly when it has been accepted with no thought of returning it' $\mathrm{p} 83^{[38]}$. This aspect of gift giving is also used in the critique on top down, North-South, post-colonial development aid [39-42], the main critique being that '...they offer aid to promote autonomy whilst buying influence for themselves' p189 ${ }^{[41]}$. The debt of the receiver is described as disempowering ${ }^{[43]}$. In twinning the attribute of reciprocity is described by authors as a core value. A consequence of the giving process is what Kowalski calls a "useful tension that will ultimately have to be released by a response at an appropriate moment" $\mathrm{p} 192^{[42]}$, it is therefore not surprising that authors mention the difficulty in balancing power relationships. The tripartite system of giving-receiving-reciprocating is described as a continuous process and participants of twinning have to take on each of these three roles in turn. The challenge of balancing their power relationship is an integral part of this process, asking for continuous (re)assessment. This also suggests that twinning is not a quick win but a process that needs to be given time. The ancient yet familiar approach of reciprocity to circumnavigate the potential disempowering effect of developing aid is an innovative aspect of twinning.

According to Gift theory, the giver does not give with the aim to receive a gift in return, 'the giving of a gift proclaims the desire for a relationship' p109 [44]. This aspect emerged in the data as the second attribute of twinning, the building of a personal relationship. The sustainability of twinning appears to be directly related to this relational investment made by both 'twins'. This is not only a personal investment, but also one of time and finances. Several authors mention equal funding to be an integral part of the twinning process ${ }^{[5,26]}$. This warrants a new approach by donors because all twins, also those from higher income countries, will benefit.

\section{TWINNING AND EMPOWERMENT}

Empowerment is seen an important strategy for the reduction of health inequities ${ }^{[45]}$. Most authors in this CA mention empowerment as an effect of twinning especially with reference to the attribute of reciprocity. There are many definitions of empowerment with contradictions and tensions including a discussion of whether empowerment is a goal or a means to an end. There is critique of its normative power 'carrying the allure of optimism and purpose' as well as that it has become a 'Buzz word' p1044 ${ }^{[46]}$. With reference to the empowerment of women, feminist critics say the term can be used 'to keep donors happy' ${ }^{[4]}$. Keeping this critique in mind, most definitions do imply a strong positive value where persons gain control over their life ${ }^{[36]}$. The definition used in this paper - 'a powerful catalyst for positive change' - includes most of the characteristics mentioned as well as being true to the comments made in the papers used for this CA. The link found between reciprocity and empowerment is not a surprise, as reciprocity directly addresses the inequality between participants as 
discussed in light of the Gift Theory. Development aid often struggles with unequal power relations, as noted by Berry: 'Working toward empowerment to reduce these inequities inevitably means addressing power relations and resource distribution' p36 [35]. Twinning deals with this issue upfront by embracing reciprocity as a core value.

\section{OTHER OBSERVATIONS}

The international/cultural aspect of the fourth attribute, that twinning is between two named organisations across cultures, can partly be the result of the search terms used. However, the attribute of 'engaging two named organisations, or people within those organisations' emerged from the data itself and is a legitimate attribute of the concept of twinning.

Boundaries of twinning such as matching, helping, one sided funding, giving training (one sided) and some characteristics of medical tourism indicate that some of the projects described in the data can be better described as training programme ${ }^{[1,17,33]}$ or study tours [28]. That absence of the four attributes of twinning and the consequence of empowerment in these articles confirm these findings.

We found that nurses and midwives use twinning as a form of collaboration more often than physicians or mixed teams. In mixed teams, the physicians were in the lead and the nurses often included later in the programme. The attributes of building a relationship and seeing twinning as a process instead of a means to an end was mentioned more often in twinning by nurses and midwives and to a lesser extent by physicians and mixed teams. This may indicate a gender difference because, despite the shift that is taking place, most nurses and midwives in the data were still women and most physicians men. Sex of the authors of the papers confirms this. This discrepancy between nurses, midwives and physicians could be because women tend to value the importance of personal relationships and the process more than men ${ }^{\left[{ }^{[8]}\right]}$ who tend to be more oriented to outcomes. However, different goals or other contextual issues could also be of influence. These, as well as the gender aspects of twinning, warrant further investigation.

\section{LIMITATIONS}

It is difficult to ascertain if all literature on twinning was included in this CA because it might not have been categorised as twinning, just as some literature on twinning included here should have been categorised as exchange or other forms of international collaboration. The latter option is more likely to have been the case because the immaturity of the concept twinning meant that the term was used freely to describe many different types of international collaboration.
Two of the papers included in this CA were written by one of the authors creating a possible bias concerning the outcomes of this CA. The authors were aware of these limitations and were alert in not allowing this to intervene with the results. Excluding congenital twins using search terms was impossible because it resulted in the exclusion of some twinning collaborations. The large number of hits on congenital twins was easy to differentiate and therefore did not pollute the results. They were excluded by hand. Table 3. reflects the information about the projects that was accessible in the published articles.

\section{CONCLUSION AND IMPLICATIONS FOR PRACTISE}

Integrating a new definition of twinning in practise clarifies the expectations of this method for participants, a necessary step for the successful implementation of twinning and for evaluating the outcomes of twinning programmes. Effective twinning has an empowering effect, which, in turn, equips healthcare professionals to play their part in SDG $3{ }^{[13]}$ Because twinning empowers both twins, it is an innovative answer to the critique that development aid promotes dependency. The challenge of attaining equality that is often experienced in twinning is an integral part of its dynamic reciprocal process. Because this process warrants continuous monitoring, (re)evaluation and adjustments, twinning is not a 'quick win'. Twinning also has the benefit of encouraging funders to revisit their vision for development: with its focus on reciprocity and equality between partners, it demands a change in standard practices.

Our new definition of twinning is an important first step in the development of this form of international collaboration, allowing it to be distinguished from others ways of working together that are more appropriately categorised as exchange, cooperation, or training programmes. Furthermore, the clear definition we offer here will facilitate new research, creating a scientific basis for twinning that will provide a richer understanding of how to better use this method.

ETHICS APPROVAL AND CONSENT TO PARTICIPATE

Not applicable.

\section{CONSENT FOR PUBLICATION}

Not applicable.

\section{AVAILABILITY OF DATA AND MATERIAL}

All data generated or analysed during this study are included in this published article. 


\section{COMPETING INTERESTS}

The authors declare that they have no competing interests.

\section{FUNDING}

There was no funding for this research.

\section{AUTHORS' CONTRIBUTION}

FC and MN conceived this study. FC initiated and coordinated the process and the study design, performed the analysis/synthesis of the data, wrote the first draft of the manuscript, and revised subsequent drafts. MN provided extensive support on the development of methodology and analysis, participated in the study design and supervised the analysis/ synthesis of data. TLJ and RdV participated in the study design. All authors edited each version and read and approved this final draft for submission.

\section{ACKNOWLEDGEMENTS}

A special thanks to Lotte Kweekel for helping in the initial literature search.

\section{REFERENCES}

1. United Nations (UN) Sustainable Development Goals. SDG 5. (2015). [cited 2020] Available from: https:// sustainabledevelopment.un.org/sdg5.

2. United Nations (UN) General Assembly. (2015). Sixty-ninth session Agenda items 13 (a).

3. United Nations (UN) Millennium Development Goals Indicators MDG's. (2000) [cited 2015]. Available from: http:// mdgs.un.org/unsd/mdg/host.aspx?Content=indicators/officiallist.htm.

4. United Nations (UN) Sustainable Development Goals. SDG 17. (2015). [cited 2020] Available from: https:// sustainabledevelopment.un.org/sdg17.

5. International Confederation of Midwives (ICM) twinning manual. (2014). Editor Moyo N. T. the Hague.

6. Jones, M. L., \& Blunt, P. (1999). 'Twinning' as a method of sustainable institutional capacity building. Public Administration and Development, 19(4), 381-402. https://doi.org/10.1002/(SICI)1099-162X(199910)19

7. World Health Organization (WHO). (2001). Guidelines for city twinning. WHO Regional office Europe. [cited 2020]. Available from: https://apps. who.int/iris/bitstream/handle/10665/107370/E74547.pdf?sequence=1

8. European Community (EC). (1999) TACIS City Twinning Programme. [cited 20157 November]. Available from: http://ec.europa.eu/agriculture/rur/leader2/rural-en/euro/p8-1-5.htm.

9. Weyreter, M. (2003). Germany and the town twinning movement. Contemporary Review, 282, 37-43.

10. Yudkin, J. S., Holt, R. I., Silva-Matos, C., \& Beran, D. (2009). Twinning for better diabetes care: a model for improving healthcare for non-communicable diseases in resource-poor countries. http://dx.doi.org/10.1136/ pgmj.2008.071589.

11. Twinning, T. Tonga - St John of God Health Care. (1992). [cited 2020]. Available from: https://www.sjog.org.au/ about-us/social-outreach/international-health/tong

12. Cord. Toilet twinning.org (2009). [cited 2020]. Available from: http://www.toilet twinning.org/twin-your-toilet-

13. United Nations Population Fund (UNFPA). (2014). The State of the World's Midwifery, a universal pathway. A woman's right to health. Editor Bernis L.D. UNFPA, ICM, WHO: New York. ISBN: 978-0-89714-026-3 Available from: http://www.unfpa.org/sowmy.

14. Morse, J. M. (2000). Exploring pragmatic utility: Concept analysis by critically appraising the literature. Concept development in nursing: Foundations, techniques, and applications, 2, 333-352.

15. Morse, J. M., PhD, C. M., Hupcey, J. E., \& Cerdas, M. (1996). Criteria for concept evaluation. Journal of advanced nursing, 24(2), 385-390. https://doi.org/10.1046/1.1365-2648.1996.18022.x.

16. International Federation of Gynaecology and obstetrics (FIGO). Three year report 2009-2012. [cited 2020] Available from: https://www.figo.org/sites/default/files/uploads//UGO/papers/Three\%20Year\%20Report\%20 2009_2012.pdf.

17. Kohi, T. W., Portillo, C. J., Safe, J., Okonsky, J., Nilsson, A. C., \& Holzemer, W. L. (2010). The Tanzania HIV/AIDS nursing education (THANE) preservice curriculum. Journal of the Association of Nurses in AIDS Care, 21(2), 9298. https://doi.org/10.1016/j.jana.2009.06.006.

18. Interagency coalition on AIDS and development (ICAD). Beyond Our Borders: A Guide to Twinning for HIV/AIDS 
Organisations. S.N.L.O. Calle Almedal, UNAIDS etc. Editor. 1999, ISBN: 0-662-28211-6: Canada.

19. American International Health Alliance (AlHA). (2010). What is twinning? [cited 2015]. Available from: http://www. aiha.com/ourstory/what-is-twinning-3/,

20. Breiddal, S. (2009). The How-To Guide to Hospice Palliative Care Twinning Projects Health Canada.

21. Busse H., Azazh A., Teklu S., Tupesis J., Woldetsadik A., Wubben R.J., Tefera G. (2013). Creating change through collaboration: a twinning partnership to strengthen emergency medicine at Addis Ababa University/ Tikur Anbessa Specialized Hospital, a model for international medical education partnerships. Acad. Emerg. Med. 20(12): p. 1310-8. doi: 10.1111/acem.12265

22. Macdonagh, Ruaraidh \& Jiddawi, Mohamed \& Parry, V. (2002). Twinning: The future for sustainable collaboration. BJU international. 89 Suppl 1. 13-7. 10.1046/j.1465-5101.2001.128.x.

23. Cadée, F., Perdok, H., Sam, B., de Geus, M., \& Kweekel, L. (2013). 'Twin2twin'an innovative method of empowering midwives to strengthen their professional midwifery organisations. Midwifery, 29(10), 1145-1150. https://doi.org/10.1016/j.midw.2013.07.002

24. Dawson A., Brodie P., Copeland F., Rumsey M., Homer C.(2014). Collaborative approaches towards building midwifery capacity in low income countries: A review of experiences. Midwifery, 30(4): p. 391-402. doi: 10.1016/..midw.2013.05.009

25. Ireland, J., van Teijlingen, E, Kemp J.(2015).Twinning in Nepal: the Royal College of Midwives UK and the Midwifery Society of Nepal working in partnership, Journal of Asian Midwives 2015;2 (1): 26-33. http:// ecommons.aku.edu/jam/vol2/iss $1 / 5 /$

26. Geus de M., Kweekel L. (2012). Royal Dutch Organisation of Midwives (KNOV), twin2twin midwives empower midwives. Editors Cadée F., Perdok H. 2012, Amsterdam. ISBN: 9789081051057.

27. Royal College of Midwives (RCM). (2015). Global midwifery twinning project. [cited 2020] Available from: https:// www.rcm.org.uk/promoting/global/projects/global-midwifery-twinning-project/.

28. Chiu, L. (2005). Motivation for nurses undertaking a post-registration qualification in Malaysia. International Nursing Review. 52(1): p. 46-51. doi: 10.1111/j.1466-7657.2004.00415.x.

29. Foster, J. (2009) Cultural Humility and the Importance of Long-Term Relationships in International Partnerships. Journal of Obstetrics, Gynaecology, \& Neonatal Nursing. 38(1): p. 100-107. https://doi.org/10.1111/j.15526909.2008.00313.x.

30. Jiang, H., Erickson, J. I., Ditomassi, M., \& Adams, J. M. (2012). Promoting a culture of international professional practice for nursing through a twinning relationship. JONA: The Journal of Nursing Administration, 42(2), 117122. doi: $10.1097 /$ NNA. 0 bo $13 e 318243384$ e.

31. Salvage, J. (2007) It Takes Two to Tango, in Nursing Without Borders: Values, Wisdom, Success Markers, S. Weinstein and A.M.T. Brooks, Editors Sigma Theta Tau.

32. Lexicon Dictionary @2020 [cited 2020]. Available from: https://www.lexico.com/definition/reciprocity,

33. Veerman, A. J. P. (2005). Twinning: a rewarding scenario for development of oncology services in transitional countries. Pediatric blood \& cancer, 45(2), 103-106.https://doi.org/10.1002/pbc.20390.

34. Qaddoumi, I., \& Bouffet, E. (2009). Supplementation of a successful pediatric neuro-oncology telemedicinebased twinning program by e-mails. Telemedicine and e-Health, 15(10), 975-982. https://doi.org/10.1089/ tmj.2009.0043

35. Berry, N. S., Murphy, J., \& Coser, L. (2014). Empowerment in the field of health promotion: recognizing challenges in working toward equity. Global health promotion, 21(4). 35-43. https://doi.org/10.1177/1757975914528725.

36. Tengland, P. A. (2008). Empowerment: A conceptual discussion. Health Care Analysis, 16(2), 77-96. https://doi. org/10.1007/s10728-007-0067-3

37. Christian, K. "Twinning”. (1978). Midwives chronicle 91(1082): p. 59.

38. Mauss, M., \& Halls, W. D. (1954). The gift: Forms and functions of exchange in archaic societies (No. 378)

39. Moyo, D. (2009). Dead aid: Why aid is not working and how there is a better way for Africa. Macmillan. ISBN 978-0-141-03118-7.

40. Chang, H. J. (2010). Bad Samaritans: The myth of free trade and the secret history of capitalism. Bloomsbury Publishing USA. ISBN10 1596915986.

41. Kowalski, R. (2011). The Gift-Marcel Mauss and international aid. Journal of Comparative Social Welfare, 27(3), 189-205. https://doi.org/10.1080/17486831.2011.595069.

42. Mawdsley, E. (2012). The changing geographies of foreign aid and development cooperation: contributions from gift theory. Transactions of the Institute of British geographers, 37(2), 256-272. https://doi.org/10.1111/j.14755661.2011.00467.x.

43. Wallerstein, N. (2002). Empowerment to reduce health disparities. Scandinavian journal of public health, 30(59_ suppl), 72-77. https://doi.org/10.1177/14034948020300031201.

44. Adloff, F. and Mau, S. (2006) Giving social ties, reciprocity in modern society. European Journal of Sociology / Volume 47 / Issue 01 / April 2006, pp 93 - 123. doi: 10.1017/\$000397560600004X.

45. World Health Organization (WHO). (1997). Office of Health Communications and Public Relations \& International Conference on Health Promotion. (4th : 1997 : Jakarta, Indonesia). The Jakarta Declaration : on leading health promotion into the 21 st century = Déclaration de Jakarta sur la promotion de la santé au XXle siécle. World Health Organization. https://apps.who.int/iris/handle/10665/63698.

46. Cornwall, A., \& Brock, K. (2005). What do buzzwords do for development policy? A critical look at 'participation','empowerment'and 'poverty reduction'. Third world quarterly, 26(7), 1043-1060. https://doi. org/10.1080/01436590500235603.

47. Mosedale, S. (2014). Women's empowerment as a development goal: taking a feminist standpoint. Journal of International Development, 26(8), 1115-1125. https://doi.org/10.1002/jid.3050.

48. Sandhu, H., Adams, A., Singleton, L., Clark-Carter, D., \& Kidd, J. (2009). The impact of gender dyads on doctor-patient communication: a systematic review. Patient education and counselling, 76(3), 348-355. https:// doi.org/10.1016/j.pec.2009.07.010 


\section{3}

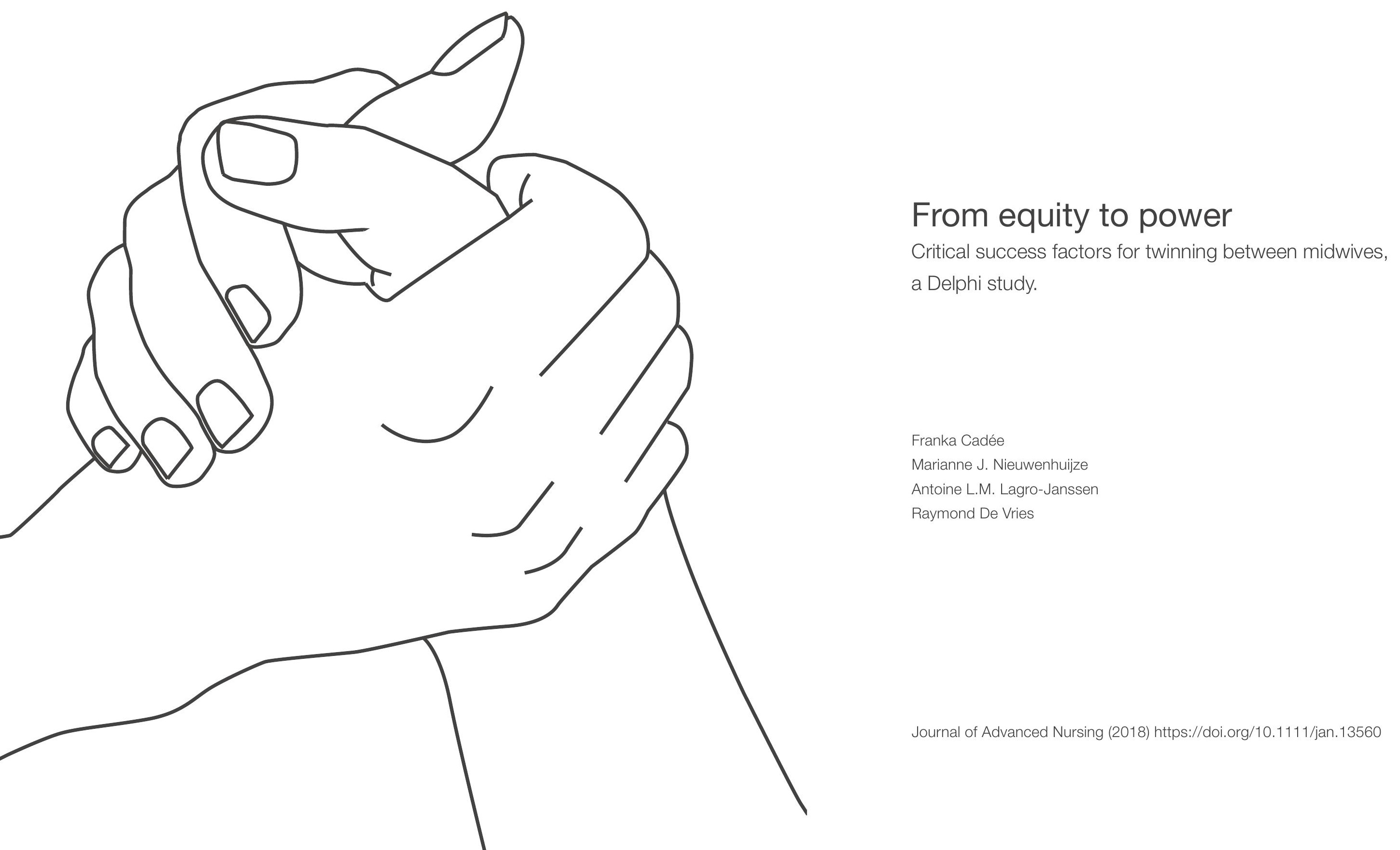




\section{ABSTRACT}

AIMS

To gain consensus for Critical Success Factors associated with twinning in midwifery.

\section{BACKGROUND}

International publications identify midwifery as important for improving maternity care worldwide. Midwifery is a team effort where midwives play a key role. Yet their power to take on this role is often lacking. Twinning has garnered potential to develop power in professionals, however its success varies because implementation is not always optimal. Critical Success Factors have demonstrated positive results in the managerial context and can be helpful to build effective twinning relationships.

\section{DESIGN}

We approached 56 midwife twinning experts from 19 countries to participate in three Delphi rounds between 2016-2017.

\section{METHODS}

In round 1, experts gave input through an open ended questionnaire and this was analysed to formulate Critical Success Factors statements that were scored on a 1-7 Likert scale aiming to gain consensus in round 2 and 3 . These statements were operationalised for practical use such as a check list in planning, monitoring and evaluation in the field.

\section{FINDINGS}

Thirty-three experts from 14 countries took part in all three Delphi rounds, producing 58 initial statements. This resulted in 25 Critical Success Factors covering issues of management, communication, commitment and values: most also focus on equity.

\section{CONCLUSION}

The Critical Success Factors formulated represent the necessary ingredients for successful twinning by providing a practical implementation framework, and promote further research into the effect of twinning. Findings show that making equity explicit in twinning may contribute towards the power of midwives to take on their identified key role.

\section{KEY WORDS}

Twinning, midwife, midwifery, Critical Success Factors, power, equity, healthcare worker, collaboration.

\section{INTRODUCTION}

The only realistic way to achieve Sustainable Development Goal (SDG) 3.1 - the reduction of the global maternal mortality ratio to less than 70 per 100,000 live births by 2030 - is to extend midwifery services and empower midwives ${ }^{[1]}$. As with any healthcare service, the extension of midwifery requires evidence of effectiveness ${ }^{[2-8]}$, supportive policy ${ }^{[9]}$ and political will [1, 10, 11]. It is clear that midwives have the skills, willpower, and passion needed to make a substantial contribution to SDG 3.1, but their potential to assume this key role has yet to be optimised. What midwives need to achieve this potential is 'power' [12]: and specifically 'power to' and not 'power over'. This perspective on power draws on Hawks' definition: 'the actual or potential ability, or capacity to achieve objectives through an interpersonal process in which the goals and means to achieve the goals are mutually established and worked towards' p745 ${ }^{[35]}$. In line with this Barrett developed a theory of power defined as 'knowing participation in change' p9 ${ }^{[13]}$ based on Rogers' Science of Unitary Human Beings ${ }^{[14]}$. There is growing evidence of the universal experience of power as viewed by Rogers and Barrett. This perception of power is specifically relevant crossculturally. Growing evidence indicates that an effective way for midwives to strengthen this type of power is through twinning ${ }^{[15-18]}$.

\section{BACKGROUND}

Despite, or perhaps because of, its emerging popularity [19], twinning is understood and implemented in a number of different ways. Twinning is unlike other forms of collaboration because of its explicit emphasis on the core value of reciprocity. In an effort to build equitable relationships, twinning encourages giving, receiving, and reciprocating as part of its method suggesting that empowerment of the participant 'twins' may be directly linked to this ${ }^{[16,20]}$. If twinning is to be a means for empowering midwives, the concept and practice of twinning needs further development.

Over the past few years midwives have gained substantial experience with twinning by participating in at least nine twinning projects between 18 midwifery organisations ${ }^{[15,19]}$. A clear and systematic method for the implementation and evaluation of these projects has yet to be developed. There is insufficient and contradictory information available about how to organise successful twinning [20]. A recent concept analysis (CA) provided an important step in addressing this gap in knowledge, offering a clear definition of twinning in healthcare - 'a cross-cultural reciprocal process where two groups of people work together to achieve joint goals' - and identifying four main attributes of twinning : 1) reciprocity, 2) building 
personal relationships, 3) dynamic process, 4) participation of two named organisations across different cultures [20]. The implementation of twinning is a dynamic process. An important step in its implementation twinning is the identification of factors critical for effective twinning, using the knowledge midwives have gained as a result of their experience with these projects. Due to the contradictory information available about how to organise successful twinning the Delphi method was used because it facilitates the transformation of opinion into consensus and therefore enhances decision making during the process of defining Critical Success factors for twinning in healthcare ${ }^{[21]}$.

The Critical Success Factors (CSF) approach offers a promising means of identifying the features of effective twinning. Defined in management literature as 'key areas of activity in which favourable results are absolutely necessary to reach goals' [22], CSF have proven to be a strategy for promoting organisational success ${ }^{[23]}$. According to Howell, CSF are practical to use and understand as they refer directly to intended results and can be used throughout a process from planning through to monitoring and evaluation. Table 1 lists four types of CSF required for effective organisations ${ }^{[22]}$.

Table 1. Rockart's four types of CSF.

\begin{tabular}{lll}
\hline Rockart's CSF categories & Explanation of category & Examples for twinning \\
\hline Industrial & Factors specific to twinning & Its reciprocal character and bridging two cultures \\
Strategic & Factors particular to the goal & Enhancing the power of midwives \\
Environmental & External factors & The context of twinning \\
Temporal & Factors arising short term & Day to day spontaneous issues \\
\hline
\end{tabular}

Identifying and implementing CSF for twinning projects can contribute towards their potential to increase the power and influence of midwives and their midwifery organisations, a critical feature for promoting respectful maternity care worldwide ${ }^{[4]}$. The identified CSF ${ }^{[2]}$ may also be useful for the successful implementation of twinning between nurses or healthcare in general. Furthermore, we think CSF may provide a practical and clear framework for further research into the effects of twinning.

\section{AIM}

To gain consensus for Critical Success Factors associated with twinning in midwifery.

\section{DESIGN}

We conducted a Delphi study between June 2016 and October 2016. This method was chosen because it offers a structured approach whereby experts of an identified group give their opinions. We started with a round of open questions followed by an iterative process of two additional rounds with statements until maximum group consensus was reached [24, 25].

\section{PARTICIPANTS}

Twinning experts were defined as midwives with personal experience in one or more twinning projects, as a participant, organiser, or evaluator between 2000 and 2016. Experts were identified through twinning projects either facilitated by, or known to the International Confederation of Midwives (ICM). ICM represents 131 midwifery organisations worldwide and uses twinning as one of its strategies to strengthen these organisations ${ }^{[15,19]}$. In addition, experts were identified using the literature search performed for a concept analysis of twinning ${ }^{[20]}$. An initial 56 experts, at least one twinning expert from every known midwifery twinning project, were invited in English by e-mail (Figure 1).

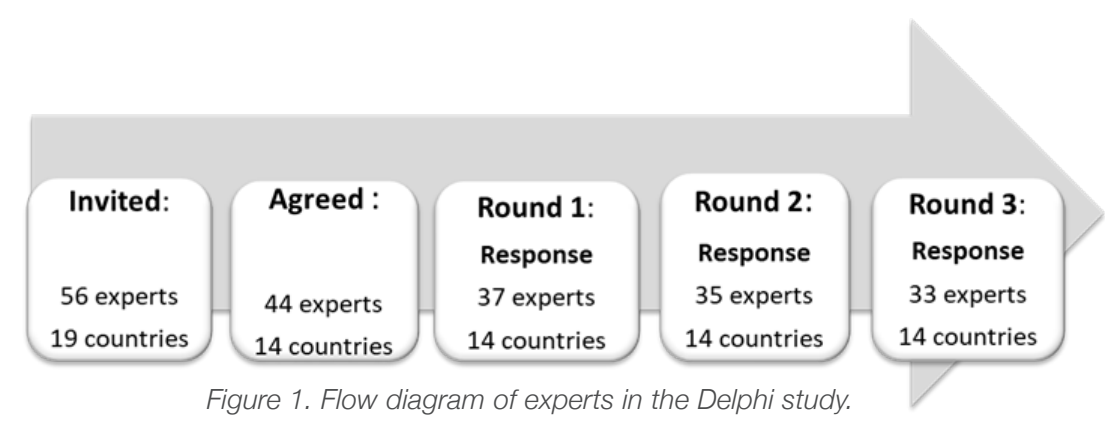

\section{DATA COLLECTION}

Experts (Figure 1) were informed of the purpose of the study by means of an invitation by e-mail. In this e-mail background information was given about twinning, referring to an earlier

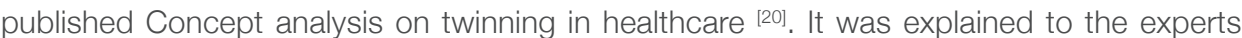
why their expertise was requested and they were informed about the scope of the Delphi method. They were also informed about the duration of the study including the expected number of rounds to reach consensus, and the estimated time investment. Experts were requested to respond by e-mail if they were willing to participate. All respondents who agreed to participate were sent a questionnaire by e-mail. To enhance the response rate and so minimise attrition, two reminders were sent at two week intervals to experts who had agreed to participate but had not yet responded, a strategy that was repeated for all Delphi rounds that were approximately one month apart per round ${ }^{[26]}$. We chose not to use internet surveys to avoid variations in internet access between experts. The possibility of responding by phone or text message was offered to all participants. To overcome these potential biases and achieve a high response rate, we made individual contact with some participants to help them stay connected, creating what McKenna terms as 'quasi-anonymity' p1224 
[25]. We did however guarantee confidentiality to all participants. To minimise attrition only experts who responded positively to the initial invitation e-mail and who completed all three rounds of the Delphi were included in the study [27].

\section{ROUND 1}

We asked experts to provide socio-demographic characteristics that may influence their opinion of twinning such as the country of the midwifery organisation they were affiliated to, their position in the project, and type and length of their twinning experience. This was followed by an open ended questionnaire where experts shared their experiences regarding factors that could either hinder or facilitate the twinning process in general and specifically for the preparation, implementation, monitoring, evaluation and sustainability of a twinning project. The attributes of twinning as identified by the concept analysis of twinning were also integrated into this open ended questionnaire ${ }^{[20]}$. Experts were encouraged to give their viewpoint as an individual 'twin' participant as well as on an organisational level, and asked to elaborate in approximately 250 words per question.

\section{ROUND 2}

The purpose of this round was to gain consensus regarding the CSF statements for twinning as well as to provide experts with the opportunity to add CSF or give comments ${ }^{[24]}$. Using the responses from round 1, CSF statements were formulated with imperatives such as 'must ', 'should' or 'only' to help experts score statements more easily on a Likert scale of 1 (totally disagree) to 7 (total agree). We used a 7 point Likert scale to allow for diversity in the answers and for the option to choose neither to agree or disagree (score 4). There is evidence that using Likert scales of $<5$ or $>7$ becomes significantly less accurate [28]. To give the experts a clearer overview of the CSF statements they were clustered into six categories. These categories were chosen on the basis of the analysed data using the different types of information given by the participants.

\section{ROUND 3}

In this round we sought to gain final consensus and/or to receive extra comments regarding the CSF statements. Except for new statements, experts were given the $\mu$ (mean) and SD (standard deviation) for the round 2 statement scores and asked to score the statements again on a 7-point Likert scale.

\section{ETHICAL CONSIDERATIONS}

Confirmation of an exemption from ethical review was given by the Medical Ethical Committee of Atrium-Orbis-Zuyd, Heerlen, the Netherlands, reference 16-N-110. In the invitation e-mail, experts were assured that participation was voluntary and that nonparticipation would have no consequence for their relationship with the research team or institution. Experts were asked to give their consent for the team to access their personal information. Participants were assured of the security of their data.

\section{DATA ANALYSIS}

\section{ROUND 1}

The answers to questions were analysed by means of a thematic content analysis using Dedoose, an online tool to assist analysing qualitative data (http://www.dedoose.com/ ). We formulated a combination of predetermined and emergent codes that were exhaustive, independent, and mutually exclusive ${ }^{[29]}$. The first author read and reread all the answers to the open ended questions, adding any missing codes. The second author monitored and checked the whole coding process. Discrepancies were discussed in the research team until consensus was reached.

The four attributes of the concept analysis of twinning ${ }^{[20]}$ were added to these codes. A content analysis of the answers to the open questions from round 1 was performed using the same online tool, Dedoose. The goal of this open questions was to explore the variation in the way twinning was implemented from one project to another, the background and context of the different organisations that twinned together. The results were clustered into categories and used to develop a questionnaire with closed CSF statements for round 2.

\section{ROUND 2}

For the results of this study to reflect consensus opinion it was necessary to define set criteria for the achievement of this consensus. In this round positive consensus was set a priori at $75 \%$ or more of the experts scoring $\geq 6$ ( $75 \%$ expert agreement) and fewer than $10 \%$ scoring $\leq 2$ (disagree); negative consensus was set at $75 \%$ or more of the experts scoring $\leq 2$ (75\% panel agreement) and fewer than $10 \%$ scoring $\geq 6$ (agree). Due to critique that the process of defining the threshold of consensus is often arbitrary, we used the relatively high cut-off of $75 \%$ expert agreement because of the relatively homogenous group of midwives who were all involved in twinning ${ }^{[24]}$. We modified statements that were close to consensus or where participants mentioned that they did not understand the statement by using their comments. These modified statements were then re-sent to the experts clustered under the same categories as in round 3 . The $\mu$ and SD for round 2 were added to each statement.

\section{ROUND 3}

In this round consensus per CSF statement was determined similarly as for round 2 and 
we calculated the $\mu$ and SD. Answers to questions and comments were analysed. CSF statements with positive consensus identified in rounds 2 and 3, were combined into themes and tabulated and ranked according to the $\mu$ and SD.

The CSF statements from round 3 were operationalised into practical CSF by removing the imperatives in the statements and joining overlapping statements. For practical use in the field, CSF statements were ordered into Rockart's four types as required for effective organisations ${ }^{[22]}$ to enable the development of a check list for planning, monitoring and evaluation purposes.

\section{VALIDITY}

We approached a diverse group of experts from both high, middle and low income countries to ensure that the sample size would be large and varied enough to reflect the aims of the study, to allow saturation ${ }^{[30,31]}$ and to provide sufficient 'information power' ${ }^{[32]}$. Because no validated questionnaire was available the questionnaire was composed making use of the literature, specifically the concept analysis on twinning ${ }^{[20]}$ and experience of the research team.

\section{FINDINGS}

We invited 56 experts from 19 countries, of which eight high income and 11 middle or low income, to participate ${ }^{[33]}$. We received a positive response from 44 experts from 14 countries, seven from high income, and seven middle or low income countries. Of the 12 other invitations, two were returned because of a wrong mail address, ten never responded to the original request after two reminders and two declined, one because of other priorities and one because she had not been involved in twinning. Of the 44 experts who agreed to participate, 37 (84\%) responded to round 1. Of these 37 respondents 35 (94\%) completed round 2 and 33 (89\%) completed round 3 (Figure 1). The final 33 experts who completed all three rounds were twinning participants $(n=13)$, organisers $(n=9)$, both participant and organiser $(n=8)$ or had a board position in a midwifery organisation involved in twinning projects $(n=3)$.

\section{ROUND 1}

Experts gave a variety of detailed responses. This information was formulated into 53 statements reflecting CSF for twinning and clustered into the following sub headings: I. Equity: Sharing in a fair way, II. Management: The organisation of the twinning project, III. Twinning within its context, IV. Twins as individuals, V. Goals: What twinning aims to achieve, VI. Communication: How twins and the management team communicate with each other and with stakeholders.

\section{ROUND 2}

Of the 53 CSF statements, there was positive consensus for 21 statements and negative consensus for one statement: 'Twins need to have the same religion' $\mu 1.29$ (SD 0.86). Of the remaining 31 statements, comments and questions by participants resulted in six new statements and seven changed statements, including one option of a question in case of a positive response. The score for the statement ' twinning projects must have at least 12 twins per twin organisation for group support to function' resulted in such a wide variation $\mu 3.13$ (SD 1.76) that we decided to drop it and replace it by the question: 'What are the maximum and minimum number of participants of a successful twinning project? This resulted in 36 statements and two questions for round 3 (Figure 2).

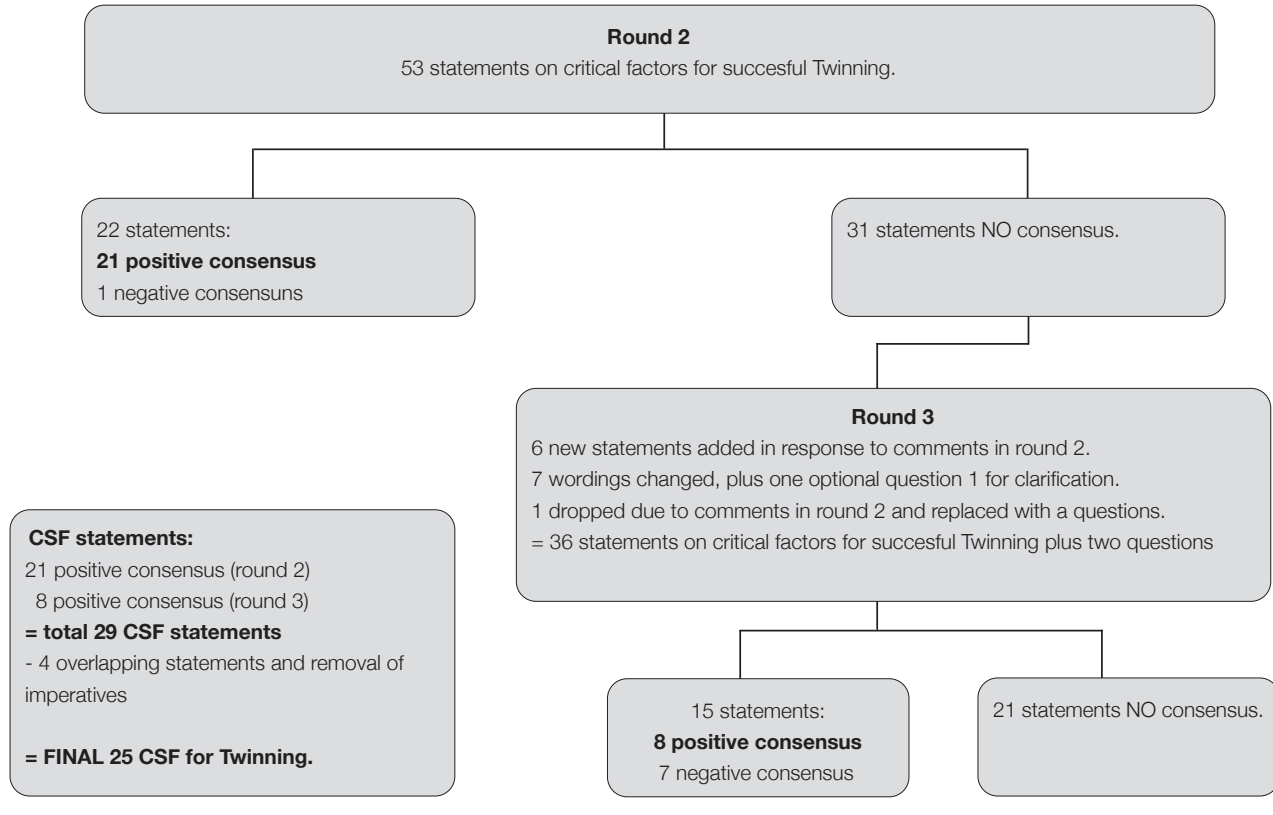

Figure 2. Delphi process for round 2 and round 3

\section{ROUND 3}

There was positive consensus for an additional eight statements leaving a total of 29 CSF statements with positive consensus after this round (Figure 2 and Table 2). The main issues covered in these positive consensus statements were: values, communication, management and commitment. The quotes below are illustrative of what the experts offered 
in their written comments:

Values: 'Cultural humility and openness to different world views are important for successful twinning...' (participant from high income country).

Communication: 'Access to the internet is the critical thing....' (participant from low income country).

Management: 'While twinning can succeed without a project leader and funding (we started that way), it was our experience that having designated staffing and funding enabled much richer input and programming' (participant from high income country).

Commitment: 'Highly interested and motivated twins will learn from the processes put in place by the twinning associations.' (participant from low income country).

Table 2. Critical Success Factors with positive consensus scored descending by $>M$.

\begin{tabular}{|c|c|c|c|c|c|}
\hline & $\begin{array}{l}\text { Critical Success Factor Statements with positive consensus } \\
\text { sorted by } \mu \text { and SD }\end{array}$ & Theme & $\mathbf{R}^{*}$ & $\mu$ & SD \\
\hline & Setting project goals has to be done by both twin organisations. & values (equity) & 2 & 6.86 & 0.35 \\
\hline & The potential of each twin has to be respected fully. & values (equity) & 2 & 6.83 & 0.37 \\
\hline & Mutual respect is a prerequisite for successful twinning. & Values (equity) & 2 & 6.73 & 0.78 \\
\hline & Goals need to be realistic for both twin organisations. & Management (equity) & 2 & 6.70 & 0.28 \\
\hline & $\begin{array}{l}\text { The twin management team must consist of representatives from } \\
\text { both twin organisations. }\end{array}$ & values (equity) & 2 & 6.66 & 0.64 \\
\hline & $\begin{array}{l}\text { All technological possibilities should be explored to facilitate } \\
\text { communication. }\end{array}$ & & 2 & 6.63 & 0.60 \\
\hline & $\begin{array}{l}\text { Every twinning project must have a monitoring and evaluation } \\
\text { plan. }\end{array}$ & management & 2 & 6.61 & 0.65 \\
\hline & Taking into account the cultural norms of your twin is essential. & Values (equity) & 2 & 6.60 & 0.80 \\
\hline & Goals need to be adapted if circumstances change. & management & 2 & 6.56 & 0.66 \\
\hline 10 & $\begin{array}{l}\text { Twins need to understand their role within the programme for } \\
\text { twinning to succeed. }\end{array}$ & management & 2 & 6.50 & 0.71 \\
\hline 1 & $\begin{array}{l}\text { All participants in a twinning project must agree about what } \\
\text { twinning stands for. }\end{array}$ & values (equity) & 2 & 6.43 & 0.78 \\
\hline 2 & $\begin{array}{l}\text { All participants in twinning need to be clear about the project goals } \\
\text { from the start of the programme. }\end{array}$ & Management (equity) & 2 & 6.43 & 0.84 \\
\hline 3 & $\begin{array}{l}\text { Motivation for the programme from start to the finish is what keeps } \\
\text { twins going. }\end{array}$ & commitment & 2 & 6.43 & 0.70 \\
\hline & $\begin{array}{l}\text { A twinning programme needs to be flexible to incorporate new } \\
\text { insights. }\end{array}$ & management & 2 & 6.40 & 0.68 \\
\hline & $\begin{array}{l}\text { Every twinning project needs to have a clear programme from the } \\
\text { start of the project }\end{array}$ & & 2 & 6.36 & 0.81 \\
\hline
\end{tabular}

\section{Table 2 continued.}

No Critical Success Factor Statements with positive consensus Theme

16 Twins need to be eager to leam

18 Managing the expectations of twins is crucial for twinning. management

19 At the basis of twinning there must be trust between twins. values

20 If twins won't give, receive, and reciprocate there is no twinning. values (equity)

21 Supportive workshops for twins about the essence of twinning management are essential.

22 All participants in twinning need to take into account the available values (equity) $\begin{array}{llll}2 & 6.22 & 093\end{array}$ resources in their twin's country.

23 The most important role of the twin management team is to keep management $\quad 2 \quad \begin{array}{lll}6 & 0.15\end{array}$ project momentum going.

24 A system for communication with stakeholders is essential. $\quad$ communication $\begin{array}{llll}2 & 6.15 & 1.12\end{array}$

25 Twins can only participate if they are prepared to receive and give values (equity) $\quad \begin{array}{llll}3 & 6.10 & 1.07\end{array}$ feedback.

26 Both twins should understand the mission, values and intent of Management (equity) $3 \quad \begin{array}{llll}3 & 6.08 & 0.99\end{array}$ each organisation and how it operates before realistic goals are established.

27 Twins must dedicate an agreed upon amount of time to the Commitment (equity) $3 \begin{array}{lll}3 & 6.06 & 1.02\end{array}$ project.

28 All participants in twinning must be equally committed to the set values (equity) $\begin{array}{llll}3 & 6.02 & 1.08\end{array}$ project goals.

29 Each twin needs to take into account the pre-existing workload values (equity) $\quad \begin{array}{llll}3 & 6.02 & 0.85\end{array}$ of their twin

*round in which consensus was reached.

Fifteen of the positive consensus statements (Table 2) focus on equity. Additionally, all 33 midwives from the 14 countries emphasized the importance of equity either by giving statements on equity high scores and/or in their written comments. This quote is illustrative of experts' comments: 'It's important to remember that equity is not the same as equality. Twins are not aiming to be similar, but to reach further in their own potential than they could have done individually and be open to respond to the opportunity that will arise'. (participant from high income country).

The CSF statements were operationalised to enable easy understanding and practical use by first combining four of the 29 consensus CSF statements that had substantial overlap in their meaning. We removed the imperatives and classified the CSF using Rockart's four types necessary for effective organisations ${ }^{[22]}$. This resulted in the final $25 \mathrm{CSF}$ for practical use in the field (Table 3). 


\section{CSF 25 Operationalised Critical Success Factors for twinning

Factors specific to twinning i.e. its reciprocal character \& bridging two

Industry: cultures.

All participants in a twinning project agree about what twinning stands for. 11 The twin management team consists of representatives from both twin 5 organisations.

Both twins understand the mission, values and intent of both organisations and 26 how it operates before goals are established.

Project goals are set by both twin organisations

Goals are realistic for both twin organisations.

Twins are prepared to receive and give feedback.

All participants are equally committed to the set project goals.

Factors particular to the goal i.e. enhancing the power of midwives.

Twins trust each other.

Twins respect each other

Twins take responsibility for their own twinning experience.

Twins are eager to learn

Twins are offered workshops about the essence of twinning.

Twins understand the project goals from the start of the programme.

The project programme is clear to all participants from the start.

\section{External factors i.e. the context of twinning.}

Environmental: A monitoring and evaluation plan is in place at the start of the programme. Twins take into account each other's cultural norms.

Twins take into account the available resources in their twin's country. Goals are adapted if circumstances change.

All technological possibilities have been explored to facilitate communication. There is a communication plan for stakeholders management.

Factors arising short term i.e. day to day spontaneous issues.

Temporal: All twins must keep motivated to participate.

The twin management team keep the project momentum going.

Twins dedicate an agreed upon amount of time to the project.

The expectations for twins are clear.

The twinning programme is flexible to incorporate new insights.

Negative consensus was obtained for seven statements focussing on the need for twins to be similar with regard to midwifery practise, organisation, education and registration. The following seven statements were indicative of the many comments made by experts about this: 'We learn from our differences. We don't have to be the same to work in partnership. However, if there are absolutely no similarities or completely different values or sense of purpose for midwifery associations, twinning maybe very challenging.' (Participant from low income country).

There were varied responses to the question about the critical minimum and maximum numbers of participants or 'twins' per project. The minimum ranged between two and 50 $($ mode $=4)$ and maximum between six and $100($ mode $=10)$ participants. Comments to this question are in line with this result: 'depends on the duration and scope of the project'. (Participant from middle income country).

\section{DISCUSSION}

The most notable result from this Delphi study is the emphasis experts put on equity, meaning 'the situation in which everyone is treated fairly and equally' ${ }^{[34]}$. This is reflected in 15 of the final 25 CSF (Table 3) and by the fact that all participating midwives, equally from rich, middle and low income countries, with their broad experience and expertise in twinning projects, mention the importance of equity in their comments.

One expert noted that 'equity does not mean being the same, as long as there is equilibrium' (participant from middle income country). This recalls the definition of twinning mentioned in the introduction: 'a cross-cultural reciprocal process where two groups of people work together to achieve joint goals'. This definition, derived from a concept analysis of twinning, identified reciprocity as a core value ${ }^{[20]}$. The leading attribute of reciprocity - which involves a system of giving, receiving and returning - is equity ${ }^{[35]}$. Even though the outcomes of twinning are not yet clearly defined, there is a probable correlation between the reciprocal character of twinning and the increased empowerment of healthcare professionals ${ }^{[20]}$. The further emphasis on equity, as a result of this Delphi study, may appear obvious because in the process of reciprocity, balancing the power of the relationship is crucial. The same balance of power is just as crucial for equity ${ }^{[36]}$. This process is not a linear but a circular one, where equity increases power, and power (to achieve objectives) increases equity. Equity is of profound importance when one wants to gain the power required to achieve the objectives of twinning: in this case, the power of midwives to play the key role in a maternity team, to optimise safe and respectful midwifery care worldwide. It must be remembered that the direct correlation between the positive effect of empowered midwives on this key role is assumed ${ }^{[15]}$ and warrants further research.

Equity is often kept implicit and thus overlooked in twinning projects ${ }^{[20]}$. The CSF from this Delphi study can be developed into an instrument for the purpose of planning, implementing, monitoring, and evaluating twinning projects in healthcare. After validation, 
such an instrument will make explicit the importance of equity in twinning projects, which can contribute towards the efficacy of twinning in healthcare.

All 25 CSF for twinning fit into one of the four types of CSF needed for successful projects as described by Rockart ${ }^{[37]}$. To our knowledge, the effect of the use of CSF in healthcare has not yet been researched. However according to Howell [23], CSF can be used in any context as long as the aim is to reach a well-defined goal. However, it must be remembered that CSF, like any other tool, are only as effective as their implementation.

Even though the participants of this study on the CSF for twinning were midwives, this study did not focus on their knowledge of practical 'hands on' midwifery, but rather on their experience in the organisational aspects of transcultural twinning. We are convinced that the CSF on twinning developed in this study are equally applicable to twinning between nurses and other healthcare professionals, or even professionals outside the sphere of healthcare such as teachers or administrators.

\section{LIMITATIONS}

Classifying CSF for twinning into Rockart's four types needed for successful projects required a certain amount of interpretation (Table 3). To minimise bias, this potential weakness was taken into account and decisions about the classification of CSF were reached after rigorous discussion and agreement within our research group.

All twinning projects known to the ICM were approached for this study. Of the 12 participants from five countries that did not respond, four were from low income countries. The research team was aware that this type of selection bias could occur for a combination of reasons, including insufficient command of English or the unreliability of the internet. It is also possible that some respondents found it challenging to be critical or give a low, or high score for cultural reasons.

The UK provided a proportionally high number of respondents, a result of the fact that each of the four countries in the UK was participating in a twinning project ${ }^{[18]}$. We believe that this overrepresentation did not affect the final results. According to the definition of 'information power', the more information the sample holds, relevant to the actual study, the fewer number of participants required ${ }^{[32]}$. We achieved high 'information power' as a result of the narrow aim of this study (determining CSF for twinning), the inclusion of experts from diverse backgrounds, and the quality and detail of the comments provided.

\section{CONCLUSIONS}

Successful twinning has the potential to increase the power of midwives ${ }^{[15,38]}$. This power specifically relates to their power to implement change ${ }^{[13]}$. This type of power is essential for midwives to take on their key role in the maternity team. The CSF determined by this Delphi study, with specific attention for the import role of equity, can be used to initiate, monitor and evaluate twinning projects. The development of a practical instrument and subsequent validation of its use can support midwives to build more successful twinning relationships. Due to the high information power of our data, the applicability of the CSF determined in this Delphi study show potential not only for twinning between midwives, but for twinning between nurses and healthcare in general. The limited amount of research into the outcomes of twinning in healthcare limits the depth of the evidence for the outcomes of twinning. Research into newly established twinning projects where an CSF instrument or a checklist based on CSF is used implemented will strengthen the evidence base.

\section{CONTRIBUTORS}

All authors participated in the study concept and design. The first author coordinated the data collection and analysis. The first and second author participated in the revision of questionnaires provided to the experts, analysis and interpretation of data, and drafted the manuscript. All authors critically reviewed the paper for important intellectual content.

\section{FUNDING}

This research received no specific grant from any funding agency in the public, commercial, or not-for-profit.

\section{CONFLICT OF INTEREST}

No conflict of interest has been declared by the authors. 


\section{REFERENCES}

1. United Nations (UN) Sustainable Development Goals. (2015). [cited 2020] Available from: https://www.who.int/ sdg/targets/en/

2. Homer, C. S., Friberg, I. K., Dias, M. A. B., ten Hoope-Bender, P., Sandall, J., Speciale, A. M., \& Bartlett, L. A. (2014). The projected effect of scaling up midwifery. The Lancet, 384(9948), 1146-1157. http:// dx.doi. org/10.1016/S0140-6736(14)60790-X.

3. Lawn, J. E., Blencowe, H., Oza, S., You, D., Lee, A. C., Waiswa, P.,... \& Mathers, C. (2014). Every Newborn: progress, priorities, and potential beyond survival. The Lancet, 384(9938), 189-205. http://dx.doi.org/10.1016/ S0140-6736(14)60496-7

4. Mwaniki, M. K. (2016). Quality in provision of maternity services: the missing link in health-care investments in LMICs? The Lancet Global Health, 4(11), e769-e770. http://dx.doi.org/10.1016/S2214-109X(16)30239-X.

5. Renfrew, M. J., Homer, C., Downe, S., McFadden, A., Muir, N., Prentice, T., \& ten Hoope-Bender, P. (2014). Midwifery: an executive summary for the Lancet's series. The Lancet, 384(1), 8.

6. Renfrew, M. J., McFadden, A., Bastos, M. H., Campbell, J., Channon, A. A., Cheung, N. F.,... \& McCormick, F. (2014). Midwifery and quality care: findings from a new evidence-informed framework for maternal and newborn care. The Lancet, 384(9948), 1129-1145. http://dx.doi.org/10.1016/ S0140-6736(14)60789-3.

7. ten Hoope-Bender, P., de Bernis, L., Campbell, J., Downe, S., Fauveau, V., Fogstad, H.,... \& Renfrew, M. J. (2014). Improvement of maternal and newborn health through midwifery. The Lancet, 384(9949), 1226-1235. http://dx.doi.org/10.1016/S0140-6736(14)60930-2.

8. Van Lerberghe, W., Matthews, Z., Achadi, E., Ancona, C., Campbell, J., Channon, A.,... \& Koblinsky, M. (2014). Country experience with strengthening of health systems and deployment of midwives in countries with high maternal mortality. The Lancet, 384(9949), 1215-1225. http://dx.doi.org/10.1016/S0140-6736(14)60919-3.

9. World Health Organization (WHO). (2015). Strategies towards ending preventable maternal mortality (EPMM). Geneva. [cited January 2020] Available from: https://www.who.int/reproductivehealth/topics/maternal_perinatal/ epmm/en/.

10. World Health Organization (WHO). (2016). Global strategy for women's, children's and adolescents' health (2016-2030). [cited 2020] Available from: https://www.who.int/life-course/partners/global-strategy/globalstrategy-key-statistics-03-11-2017.pdf.

11. United Nations Population Fund (UNFPA). (2014). The State of the World's Midwifery, a universal pathway. A woman's right to health. Editor Bernis L.D. UNFPA, ICM, WHO: New York. ISBN: 978-0-89714-026-3 Available from: http://www.unfpa.org/sowmy.

12. Lopes, S. C., Nove, A., ten Hoope-Bender, P., de Bernis, L., Bokosi, M., Moyo, N. T., \& Homer, C. S. (2016). A descriptive analysis of midwifery education, regulation and association in 73 countries: the baseline for a post2015 pathway. Human resources for health, 14(1), 37. doi: 10.1186/s12960-016-0134-7.

13. Caroselli, C., \& Barrett, E. A. M. (1998). A review of the power as knowing participation in change literature. Nursing Science Quarterly, 11(1), 9-16. https://doi.org/10.1177\%2F089431849801100105.

14. Rogers, M. E. (1994). The science of unitary human beings: current perspectives. Nursing Science Quarterly,
7(1), 33-35. https://doi.org/10.1177/089431849400700111.

15. International Confederation of Midwives (ICM) twinning manual. (2014). Editor Moyo N. T. the Hague.

16. Cadée, F., Perdok, H., Sam, B., de Geus, M. \& Kweekel, L. (2013). 'Twin2twin'an innovative method of empowering midwives to strengthen their professional midwifery organisations. Midwifery, 29(10), 1145-1150. https://doi.org/10.1016/.midw.2013.07.002

17. Ireland, J., van Teijlingen, E, Kemp J. Twinning in Nepal: the Royal College of Midwives UK and the Midwifery Society of Nepal working in partnership, Journal of Asian Midwives 2015;2 (1): 26-33. http://ecommons.aku. edu/jam/vol2/iss $1 / 5 /$

18. Royal College of Midwives (RCM). (2015). Global midwifery twinning project. [cited 2020] Available from: https:// www.rcm.org.uk/promoting/global/projects/global-midwifery-twinning-project/.

19. International Confederation of Midwives (ICM). (2014). New Twinning Workshop. [cited 2015] Available from: http://www.internationalmidwives.org/news/?nid=169.

20. Cadée, F., Nieuwenhuijze, M. J., Lagro-Janssen, A. L. M., \& De Vries, R. (2016). The state of the art of twinning, a concept analysis of twinning in healthcare. Globalization and health, 12(1), 66. doi: 10.1186/s12992-0160205-5.

21. Hasson, F., Keeney, S., \& McKenna, H. (2008). Research guidelines for the Delphi survey technique. Journal of advanced nursing, 32(4), 1008-1015. https://doi.org/10.1046/j.1365-2648.2000.t01-1-01567.x.

22. Bullen, C. V., \& Rockart, J. F. (1981). A primer on critical success factors. Massachusetts Institute of Technology: Sloan School of management. Massachusetts.

23. Howell, M. T. (2009). Critical success factors simplified: Implementing the powerful drivers of dramatic business improvement. Productivity Press, New York.

24. Diamond, I. R., Grant, R. C., Feldman, B. M., Pencharz, P. B., Ling, S. C., Moore, A. M. \& Wales, P. W. (2014). Defining consensus: a systematic review recommends methodologic criteria for reporting of Delphi studies. Journal of clinical epidemiology, 67(4), 401-409. http://dx.doi.org/10.1016/i.jclinepi.2013.12.002.

25. McKenna, H. P. (1994). The Delphi technique: a worthwhile research approach for nursing?. Journal of advanced nursing, 19(6), 1221-1225. https://doi.org/10.1111/j.1365-2648.1994.tb01207.x.

26. Keeney, S., Hasson, F., \& McKenna, H. (2006). Consulting the oracle: ten lessons from using the Delphi technique in nursing research. Journal of advanced nursing, 53(2), 205-212. https://doi.org/10.1111/j.13652648.2006.03716.x.

27. Sinha, I. P., Smyth, R. L., \& Williamson, P. R. (2011). Using the Delphi technique to determine which outcomes to measure in clinical trials: recommendations for the future based on a systematic review of existing studies. PLoS medicine, 8(1). doi: 10.1371/journal.pmed. 1000393

28. Johns, R. (2010). Likert items and scales. The University of Sheffield. [cited 2020]. Available from: https:// pdfslide.net/documents/likert-items-and-scales-the-university-of-sheffield-filelikertfact1-survey.htm.

29. Burla, L., Knierim, B., Barth, J., Liewald, K., Duetz, M., \& Abel, T. (2008). From text to codings: intercoder reliability assessment in qualitative content analysis. Nursing research, 57(2), 113-117. doi: 10.1097/01. NNR.0000313482.33917.7d

30. Morse, J. M. (1995). Exploring the theoretical basis of nursing using advanced techniques of concept analysis. 
Advances in Nursing Science. 17(3): p. 31-46. https://doi.org/10.1097/00012272-199503000-00005.

31. Mason, M. (2010). Sample size and saturation in PhD studies using qualitative interviews. In Forum qualitative Sozialforschung/Forum: qualitative social research (Vol. 11, No. 3). http://dx.doi.org/10.17169/fqs-11.3.1428.

32. Malterud, K., Siersma, V. D., \& Guassora, A. D. (2016). Sample size in qualitative interview studies: guided by information power. Qualitative health research, 26(13), 1753-1760. https://doi.org/10.1177/1049732315617444.

33. World-Bank. New country classifications by income level: 2017-2018. [cited 2017] Available from: https://blogs. worldbank.org/opendata/new-country-classifications-income-level-2017-2018.

34. Hawkins, J. and Le Roux S. (1986). The Oxford reference dictionary. 1986, Oxford University Press, USA.

35. Crespo, R. F. (2008). Reciprocity and practical comparability. International Review of Economics, 55(1-2), 13-28. doi: 10.1007/s12232-007-0028-z.

36. Hawks, J. H. (1991). Power: a concept analysis. Journal of Advanced Nursing, 16(6), 754-762. https://doi. org/10.1111/1.1365-2648.1991.tb01734.x.

37. Rockart, J. F. (1979). Chief executives define their own data needs. Critical success factors. Harvard Business Review, 1979. Harvard business review, 57(2), 81-93.

38. Wallerstein, N. (2002). Empowerment to reduce health disparities. Scandinavian journal of public health, 30(59_ suppl), 72-77. https://doi.org/10.1177/14034948020300031201. 


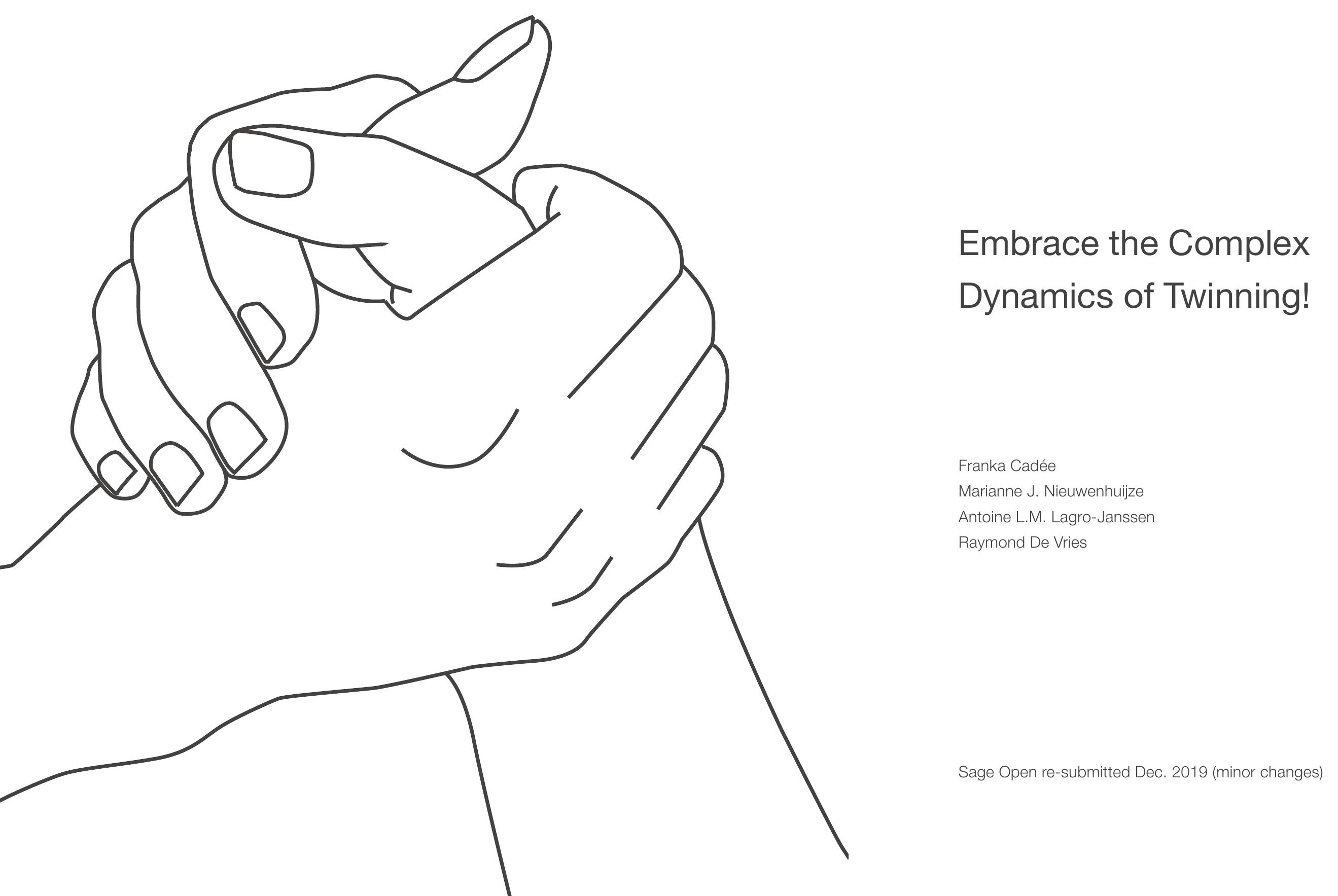




\section{ABSTRACT}

The professional growth of midwives, essential for optimising midwifery leadership globally, can be enhanced through twinning collaborations. How twinning promotes growth is unclear. This case study explores how professional growth is affected by cultural differences between twins. We used a longitudinal qualitative design including data from open-ended questionnaires and focus groups. These data were analysed using content analysis. Our findings show that cultural differences were capable of both hindering and facilitating professional growth. Within the complex dynamics of twinning, professional growth was facilitated by twins' preparedness to bridge cultural differences. Common goals positively influenced this process. Friction was more likely, and professional growth was hindered, when midwives were unprepared to bridge cultural differences. To optimise professional growth through twinning, we recommend a clear focus on common goals and consideration of the interaction between the length of a project and the extent of the cultural differences between twins.

\section{KEYWORDS}

Midwifery, Capacity and Development, Transcultural, Power, Empowerment, Professional, Multiculturalism.

\section{INTRODUCTION}

Although global maternal mortality decreased by almost 44\% between 1990 and 2015, each day more than 850 women die during pregnancy, childbirth or soon after [1]. In response to this problem, the World Health Organization (WHO) has launched strategies to implement appropriate care for women and their families that is neither 'too little too late or too much too soon' [1-4]. These include the deployment of WHO guidelines on antenatal and intrapartum care, ensuring universal access to sexual and reproductive health-care services, and expanded attention on a positive birth experience for women ${ }^{[5-9]}$. Trained, registered, and confident midwives, as defined by the International Confederation of Midwives (ICM), are a vital component of the effort to increase women's positive pregnancy and birth experiences and reduce maternal and new-born mortality and morbidity ${ }^{[5,10-12]}$. As a predominantly female profession serving women, gender-based inequities for midwives

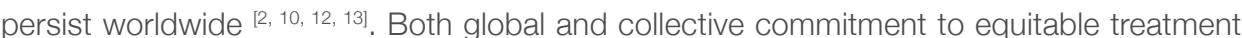
of midwives is required if midwives are to take the leading role that is envisioned for them $[14,15]$. This can be achieved by legalising midwifery, educating and registering midwives, addressing economic pay gaps, and encouraging midwives to unify their voice and take on leadership positions ${ }^{[15-18]}$.

One way to strengthen midwives' leadership capacity is through twinning, a programme known to enhance the professional growth of participating twins, thus enabling them to take leadership roles ${ }^{[19-21]}$. Twinning helps people to learn and transform by exploring the unknown and coming out of their comfort zone ${ }^{[2]}$. In this article, we use the term professional growth to include personal growth which incorporates the ability to be impactful as a person involved in decision-making at local, regional, national or global levels.

The formal definition of twinning is: "a cross-cultural, reciprocal process where two groups of people work together to achieve joint goals" p66 ${ }^{[23]}$. WHO stimulates the implementation of twinning partnerships through the promotion of "Collaboration, co-development and sharing of both tacit and explicit knowledge" p62 [19]. The ICM promotes twinning because it encourages leadership skills amongst midwives through reciprocal learning ${ }^{[23]}$. The number of twinning initiatives worldwide is rapidly increasing, including: the Midwifery Associations of Tanzania and Canada, Japan and Mongolia, the UK and Uganda, UK and Nepal, the Netherlands and Sierra Leone, the Netherlands and Iceland, and Ghana and Sierra Leone $[20,24-29]$

Common to all these projects is the dynamic of the interaction across two cultures. There is a lack of systematic exploration into this dynamic and how it influences professional growth. 
In this article we investigate the dynamics of a twinning project with midwives from Morocco and the Netherlands. These midwives were supported while they worked in groups of 2-4 twin pairs on small-scale products. These products served two main purposes, first and foremost the joint products actively challenged twins to work collaboratively and placed them in a position to need to communicate cross-culturally. Secondly, the end-products aimed to improve midwifery care in each country.

Throughout the project, twins participated in workshops and took part in several exchange visits, where they stayed at each other's homes and shared their work and private life. All twins attended the ICM Midwives Congress in Prague in 2014. Other contact was through phone, Skype, email, Facebook and WhatsApp.

The aim of this study was to gain more insight into the dynamics of twinning between midwives from Morocco and the Netherlands, with specific attention to the impact of working cross-culturally on their professional growth.

\section{METHODS}

This case study is based on longitudinal qualitative data collected between 2013-2017 from the 36 midwives participating in the Moroccan-Netherlands twinning project. Participating midwives identified themselves as 'twins' and are referred to as such throughout this article.

\section{PARTICIPANTS}

Initially 19 pairs of midwives were recruited from both midwife associations through email and individual selection. We selected the twins based on their various midwifery backgrounds in order to cover all the different positions midwives can take in their professional field. All participants worked or studied full time in the field of midwifery. Twins were paired based on their midwifery focus-area, age and experience: students to students, teachers to teachers, researchers to researchers, practising midwives to practising midwives, etc. The presidents of both professional organisations were also twinned. Two Dutch twins were of Moroccan origin. All twins came from diverse geographical areas in both countries. All Moroccan twins had a good demand of French and Arabic with some English. All Dutch twins had a good demand of English and Dutch with some French. There were two tri-lingual midwife translators present during all workshops and exchange visits.

\section{DATA COLLECTION AND ANALYSIS}

To gain a deeper understanding, we collected survey and focus group data. We started with a baseline open-ended survey in 2013 to ascertain twins' expectations and what they aimed to achieve. This baseline survey was completed either online or on paper at the end of the kick-off workshop which was held at the start of the project.

At mid-term in 2015 and end 2017, we conducted focus groups to give twins ample space to explore, discuss and reflect on their experiences, progress and achievements. Because of the projects' multi-cultural nature and language issues within the group, focus groups per country were chosen as the best way to explore experiences and sentiments in depth [30]. Focus groups took place in the twins' country of residence. Twins were randomly divided into four even groups, two in Morocco and two in the Netherlands. The first and second author and, in the case of Morocco, a professional interpreter were present at all focus groups. We formulated the questioning route using the information from the project monitoring and evaluation and from the baseline questionnaire. All methods were aimed to give enough space to both positive and negative experiences. Examples of these questions during the focus groups were: 'please tell me your personal stories; What did the project bring you?; What could you have done without?; What did you miss? Etc.

In Morocco the focus groups were held in both French (for twins) and English (for researchers) in the presence of a professional interpreter who transcribed the recordings into English. In the Netherlands, the focus groups were held in Dutch, and the recordings were transcribed into Dutch and then simultaneously analysed and translated into English. The data from the initial survey and the focus groups were analysed by means of a thematic content analysis using the online tool Dedoose ${ }^{[31,32]}$. We used a combination of predetermined codes based on the response to the open-ended questions of the baseline survey that included cultural exchange, midwifery skills, and equity, and emergent codes such as reciprocity, professional growth and religion. These codes were exhaustive, independent, and mutually exclusive ${ }^{[32]}$.

The first author coded the responses to the base-line survey first. Subsequently, she listened to the recordings, read and reread all the transcribed focus groups and analysed these making use of the same codes identified during the base-line survey and adding new emergent codes. The second author monitored and checked the coding process. It was not possible to identify individual twins from the recordings of the transcribed and translated focus groups, and for this reason illustrative quotes are not identified with a participant number but only with their nationality, $\mathrm{M}$ for Moroccan and $\mathrm{D}$ for Dutch. Care was taken to give a broad reflection of the themes discussed. There is no reason to believe that the four twins who did not attend the focus groups did so specifically to 'not voice' their views. We therefore assume that all views on the twinning project were represented. 


\section{RIGOUR AND REFLECTIVITY}

We used the COnsolidated criteria for REporting Qualitative research (COREQ) Checklist for the reporting of this research ${ }^{[33]}$. The project lead managed the twinning project and simultaneously compiled, gathered and analysed the research data from the surveys and focus groups. The research team considered the consequences of the double role of the project manager / first author. The advantage of being part of the project process was that this facilitated the organisation of focus groups, and gave the first author familiarity with the project, its settings, and deeper insight into what was going on. There was no indication that this jeopardised mutual trust and open atmosphere during the focus groups. To counterbalance potential subjectivity in observation and analysis, the second author, who was not involved in the project in any way, was present at all the focus groups and provided a critical eye with more distance. The full research team took care to identify and debate any potential pitfalls of the double role. Discrepancies were discussed until consensus was reached.

\section{ETHICAL CONSIDERATIONS}

According to the rules governing research with human subjects in the Netherlands (https:// english.ccmo.nl/), this research did not require ethical approval because of its non-invasive nature. Participating midwives were part of a twinning project and explicitly informed and asked for consent to use the data from all the surveys and focus groups at the onset of the project and again before these were performed. The twins were made aware that only quasi anonymity could be guaranteed because of the small size of the group. In Morocco, no medical ethical approval was required for this research because the participants were not medical patients. Permission was granted by the midwives themselves as well as by the Board of the Moroccan association of midwives. All participating midwives were members of the association.

\section{FINDINGS}

Nineteen Dutch and 19 Moroccan participants, making 19 pairs, were recruited into the project. Respectively 16 and 19 completed the baseline open-ended survey. Personal reasons accounted for three midwives not completing the survey, but they did participate in the mid-term and end focus groups. One twinned couple dropped out after the first year because of an unbridgeable issue leaving 18 twin couples to complete the project and participate in the midterm and end term focus groups.

At mid-term, half of the twins had been on an exchange visit to their twin's countries and all twins had attended the 2014 ICM Triennial Congress. The mid-term focus groups took place within 6 months of these activities. One Moroccan midwife was on maternity leave and three Dutch midwives indicated that they had other priorities and chose not to attend. During the end focus groups one Dutch twin was on holiday, but all the others participated.

Throughout the four-year project period, all twins participated actively and appeared eager to share their experiences. The data revealed that many twins experienced language barriers as a hindrance to varying degrees. There were a few bilingual twins who had no language issues and some who were proud to have improved either their French or English.

Findings on the dynamics of twinning are described in two parts. The first presents the initial motivation and achievements as voiced by twins through time: at baseline (2013), midterm (2015), and the end of the project (2017). In the second part, we offer a wider picture, with specific attention for the role of cross-cultural collaboration on twins' professional growth.

\section{FROM INITIAL MOTIVATION TO FINAL ACHIEVEMENTS}

\section{INITIAL MOTIVATION - BASELINE 2013}

Three main themes - related to the motivation of the twins for participating in the project emerged from the analysis of the base-line data. These were: to improve maternal health, gain professional growth and experience a cultural exchange.

Wanting to make a positive contribution to maternal health was shared with equal enthusiasm by all twins. Most Moroccan twins voiced wanting to contribute to the improvement of the health of Moroccan women, whilst many Dutch twins referred to improving the health of the Moroccan immigrant population in the Netherlands. Twins' focus on professional growth, including their leadership and midwifery skills, was a clear motivator. Most Moroccan twins specifically linked professional growth to midwifery skills, protocol development, and strengthening their midwifery association. Fewer Dutch twins voiced the importance of professional growth for themselves at the onset of the project, nor did they mention their midwifery association. Wanting to experience each other's cultural differences in the broadest sense during the exchange was described with excitement and curiosity by all (Table 1). 


\section{Improve maternal healt}

M: "Moral satisfaction that I can contribute directly or indirectly to the health of women and new-borns in Morocco." D: "Gain more insight into why our (Moroccan) population has less good outcomes in the Netherlands and learn ways to deal with this."

Professional growth

D: "... .learning from each other, from my twin and from the group."

M: "...learn from Dutch experiences in the field of reproductive health, ...and protocol support.' 'Association management, ... fundraising and increasing the numbers of members of our association.

Cultural exchange

M: "... a space to exchange knowledge and culture."

D: "Enriching myself with language and culture, getting to know new people with new ideas, and insights about collaboration and learning. Refreshing."

\section{GAINING MOMENTUM - MIDTERM 2015}

By midterm twins had gained more collaborative experience through working on their joint group products (see list, Table 2). Most twins gave at least 90\% of the agreed time, an average of 10 hours a month, to their group project.

Table 2. Products developed collaboratively by twins.

$\checkmark \quad$ Workshop for women about pain management in labour.

$\checkmark$ Stress management training for student midwives.

$\checkmark \quad$ Instruction about birthing positions for midwives.

A qualitative interview study of knowledge of hypertensive disorders in pregnancy of Moroccan women in Morocco and in the Netherlands: (https://doi.org/10.1186/s12884-018-1980-1).

$\checkmark$ Activities to raise the awareness about midwives for women in rural areas in Morocco.

$\checkmark$ Antenatal classes for rural women in Morocco.

$\checkmark \quad$ Flyers about antenatal care and screening for abnormalities.

Film to encourage Dutch girls of Moroccan origin to become midwives (https://www.verloskunde-academie.n// verloskunde-studeren/)

Twins had clearly become more conscious of each other's cultural contexts by midterm. Most twins voiced sentiments like being part of a 'global midwifery family' and linked this directly to the 2014 ICM Triennial Midwifery Congress in Prague. This international gathering of more than 4000 midwives was attended by all twins and they reported it to be deeply inspiring. All twins had communicated with each other electronically and half of the group had been on an exchange visit to their twins' country. Improving maternal health remained a key motivator that united twins in their endeavours. However, their active collaboration did not translate into the same kinds of professional growth for all twins. Most Moroccan twins voiced a steady increase in their professional growth. Many Dutch twins did not refer to growth but instead to 'letting go' of initial expectations and saw professional growth in their twins rather than in themselves. Maintaining the good reputation of Islam and Muslims was reported by several Moroccan twins (Table 3).
Table 3. Illustrative quotes from Moroccan (M) and Dutch (D) twins in 2015 Focus Groups.

\section{Improved maternal healt}

M. Tthink now the Dutch miawives ... (have been) with Moroccans (from Morocco)...they will communicate better with Moroccans (living in Holland)."

D: "What I found strange were the nasty videos shared (in the WhatsApp group) where women abused their power over men...then I realised the emancipation (of women) they are still working on..."

\section{Professional growth}

M: “... (it was) an opportunity for us to develop our self-confidence..."

D: "I have let go a bit....because I just don't know where we are in the process. Six months ago I felt we were participating in the same project...it costs too much (energy) the way we do it now does not work...'I'l see what happens..."

\section{Cultural exchange}

M: "I think Moroccans are the best representatives of Islamic and Arab culture. I am glad that we made some bad ideas about Arabs, change.

D: "... how they live together and support and help each other. That is very different here, and it can be oppressive, but also something beautiful."

REFLECTIONS ON ACHIEVEMENTS - END 2017

All twins were satisfied, albeit to different degrees, with the outcomes of the project. They were specifically pleased with the midwifery products described in Table 2, which were exhibited both in Morocco and in the Netherlands. The Moroccan twins were satisfied with their professional growth, reporting a steady increase in their leadership capacity. With a few exceptions, the tone amongst Dutch twins was more negatively critical, and some said that twinning had not influenced their growth positively. Moroccan twins expressed pride in their own culture and the opportunity to gain international visibility. For their part, some Dutch twins became more critical of Moroccan culture, while others remained curious (Table 4).

Table 4. Illustrative quotes from Moroccan (M) and Dutch (D) twins in 2017 focus groups.

\section{Improved maternal health}

D: ".... and in Morocco I noticed that it is about reclaiming what has got lost (about vertical birth). Those (Moroccan) women knew so much..."

bith for you is a normal thing. it's so natural, but in our place it is more medicalised. After the twinning we try to be like that. We try not to medicalise anymore."

Professional growth

M: "Twinning... enabled us to be associated with the Health Ministry, in order to promote programmes for mother and child health care..."

D. " Tcould not i.."

Cultural exchange

Dit "Whave gained from it is more understanding of the Moroccan and Muslim culture."

M: "We should be given a sort of chart so that everyone knows their limits...For example...twins should know what is possible to say, to do, how to respect the other, that's it:"

(1)

D: "I know a Moroccan woman who is a lesbian, well that is a drama (in Morocco)."

A few twins mentioned the importance of having gained a friend. By the end of the project most Dutch twins experienced the relationship as collegial, whereas most Moroccan twins 
spoke of the sisterly aspect of their relationship using terms like warmth and love. Here too, exceptions existed. A few Dutch twins mentioned their love for their twin and a few Moroccan twins said they had no inclination to meet their Dutch twin again. When asked if twins would want to be part of the project again, including facing the challenges, both groups became similarly animated and emotional at the thought of missing out on the experience they had shared, seemingly forgetting their earlier mentioned reservations.

\section{THE WIDER PICTURE}

During the analysis of the midterm and final focus groups, two subthemes emerged under the theme cultural exchange, giving additional insight into the role of cross-cultural collaboration and its influence on the professional growth of the twins. Both themes relate to the differences in culture and the responses those differences engendered. The first theme centres on how the differences in culture afforded an opportunity for professional growth. The responses to these cultural differences were not uniformly positive. A second theme found in our data were accounts of irritation and unresolved differences.

Whilst the idea of a cultural exchange was a motivator for most twins to take part in the project, there was no mention of the possible positive and negative dimensions of working across cultures during the baseline in 2013. Twins showed no awareness of the roles that cultural differences might play in their collaboration. By 2015 this had changed, with all twins acknowledging cultural differences. Most twins describe these cultural differences as a worthwhile challenge that could potentially facilitate their professional growth. However, for some Dutch twins, bridging cultural difference was more of a concern, a problem caused by the 'other' culture, not their own.

During the midterm focus groups in 2015, Dutch midwives were more likely to mention the negative consequences of cultural differences, expressing irritation and using evasive language during discussions of sensitive issues (observations of first and second author). A Moroccan twin mentioned the seeming inhospitality of a Dutch twin and Dutch twins pointed to the lack of transparency and hierarchical behaviour within the group of Moroccan twins. When asked to elaborate both Dutch and Moroccan twins agreed that Moroccan culture was more focussed on the community and the Dutch more on the individual (Table 5).

By 2017 the tone amongst Dutch twins became more pessimistic to the extent that the adverse consequences of cultural difference emerged as the dominant theme, specifically in one focus group characterised by negativity. A few Dutch twins contacted the first author after this focus group to express their disappointment with this negativity. They had felt unable to mention this during the focus group itself. Concerns about hierarchy became more prominent amongst Dutch twins and focussed more on Moroccan society in general. Some of the Dutch twins said that Moroccan twins did not speak their minds openly because of group pressure, going as far as labelling this as dishonesty. Within the Moroccan focus groups, the negative aspects of cultural difference was mentioned a few times, but did not dominate the discussions. Instead Moroccan twins saw these sorts of problems as the inability of individual twins to accept and trust each other.

A few twins considered a lack of equity and reciprocity between them demotivating. Several Dutch twins voiced that they gave more than they received during the project whilst Moroccan twins voiced concerns about equitable support for them due to reduced capacity on the Moroccan side. (Table 5, 2017).

Table 5. Illustrative quotes from Moroccan (M) and Dutch (D) twins on cultural differences in two Sub-themes.

\section{Subtheme 1. Cultural differences that afforded an opportunity}

2015 D: "It is something in their culture, and in ours, that does not match." M: "Moroccan people are more sociable..."

2017 M: "...she had that stereotype....and then when she (took part in) the project, she approached the population through midwives, she got to know her twins, my family, my environment... and I think it made her think twice about the Moroccan population..."

D: "They are not like us. When the project (proposal) is sent they may add a few things but will take nothing of ours out...I said it was there to discuss. But they said no, if you wrote it we will leave it in..."

Subtheme 2. Culture differences that remained irritating and unresolved

2015 D: "... they seem to want to have power (over each other ), when I would think they should combin their strengths...

M: "...for us twins we feel that some from the other side are dominant. Like they're imposing things.".

2017 M: "I felt ignored...even more, not respected... she completely demeaned me, I am talking about respect (and) trust."

D: "They (Moroccans) impose things on each other... We (Dutch) learn to discuss with each other, that is a modern thing. Here in the Netherlands we can discuss and even be enemies and still be fine. That is not the case in Morocco, they impose things and that is the difference."

Overall, a noticeable change emerged over four years amongst twins, but this change appeared to be moving in opposite directions. At the onset twins did not mention cultural differences as an item. By 2015 most Moroccan twins noticed cultural differences, saying that some Dutch twins were less tolerant than Moroccan twins, and by 2017, they reported a few negative results of cultural differences, but associated this with individual behaviour. Most Moroccan twins were satisfied that the results of the project matched most of their expectations and they reported a steady increase in their professional growth. A few Moroccan twins did disengage from active participation in the project out of personal frustration, and they reported not having experienced professional growth. For many Dutch 
twins, a positive view of cultural differences, expressed at mid-term, transformed to a more negative view by 2017, with many indicating that their professional growth had remained unchanged. Quite a few Dutch twins questioned the satisfaction of Moroccan twins about their achievements, labelling it as nothing more than socially acceptable behaviour. Some Dutch twins were satisfied with their achievements, even if it did not always match their expectations. These same twins also voiced having experienced professional growth.

\section{DISCUSSION}

This study of a twinning project between 18 pairs of Moroccan and Dutch midwives reveals the complex dynamics that make up twinning. The data demonstrate that the experiences of twins changed over time and was positively influenced by sharing common goals. Had the findings been solely based on focus groups at the end of the project, these changing dynamics would not have become apparent. By the end of the project it became clear that cultural differences were capable of both hindering and facilitating the professional growth of twins. Reflecting on these findings, we make three noteworthy observations after which we will discuss the place of culture in the dynamics of twinning.

\section{THREE OBSERVATIONS}

First, we observed that twins agreed that Moroccan culture was more focussed on community and the Dutch more on the individual. This has been described in the literature as a difference between individualist and collectivist cultures, with the interest of the individual prevalent in the first, and the interest of the group dominant in the other [34]. In our study, this was reflected in the concerns the Dutch twins had about Moroccan twins being 'collectivistic' and therefore not openly speaking their minds, and reporting professional growth when there was none, in order to protect the image of the group.

Ironically, during the focus groups the individual Moroccan twins appeared free to voice diverging views whilst some of the Dutch twins reported feeling uneasy about bringing up negative messaging during their own focus group. Thus, it appears that group pressure also affected Dutch twins, suggesting that the work of the Hofstedes, referred to above, cannot fully account for the variation and complexity reflected in our data. The tendency to label the satisfaction of Moroccans as a desire to be socially acceptable and to ascribe honesty and transparency to the more individualistic culture of the Dutch is a recognised phenomenon in collaborations between people of different cultures and can best be explained as judgements based on one's own cultural values ${ }^{[35]}$.
Our second observation is a response to the Dutch twins' report that they experienced little professional growth. Other studies show that the 'Northern side' in international collaborations generally learns more than is voiced initially ${ }^{[38]}$. For example, it seems Dutch twins failed to see their learning to 'let go' as professional growth. 'Letting go' can be seen as an important life lesson, an aspect of both personal and professional growth.

Finally, the changing global context likely had an influence on the twins. Most importantly, the growing anti-Islamic sentiments during the time-span of the project affected the relationship between members of both groups. This was most visible in the pride in, and protection of, their religion and culture on the part of the Moroccans and the dismay over the lack of freedom of the Moroccan twins that was expressed by the Dutch participants.

\section{THE PLACE OF CULTURE IN THE DYNAMICS OF TWINNING}

As we have seen, the cultural context was referred to by all twins in one way or another and was given much attention during the twinning project workshops. When defining culture, Schein (2010) describes it as the agreed upon behaviours within a group that are passed on to the next generation. His approach to culture helps explain, for example, why one twin may be less confrontational or the other more direct in their feedback, which allows us to contextualise certain comments by twins ${ }^{[35]}$. An understanding of the way the culture directs behaviour is important for successful twinning [23, 37]. Adler and Gundersen (2008) are critical about any attempt to define culture because, they believe, the effort results in overgeneralisations that are meaningless when we refer to individuals within a culture. We saw this in our study in the individual variation present amongst twins, regardless of their cultural background ${ }^{[38]}$.

Following from Schein's perspectives on culture, the Hofstedes developed tools to understand culture in order to minimise its disruption on multinational corporations ${ }^{[34]}$. Their acculturation curve helps to explain the acculturation process of immigrants and, in our case, it offers insight into the process we witnessed. We saw the initial general curiosity and enthusiasm of twins in 2013 (Euphoria stage) turn into apprehension and concern in 2015 (culture shock stage) and end in 2017 in either friction (a), bridging a culture-gap (b) adapting as a native (c), stable state stage. Figure 1. 


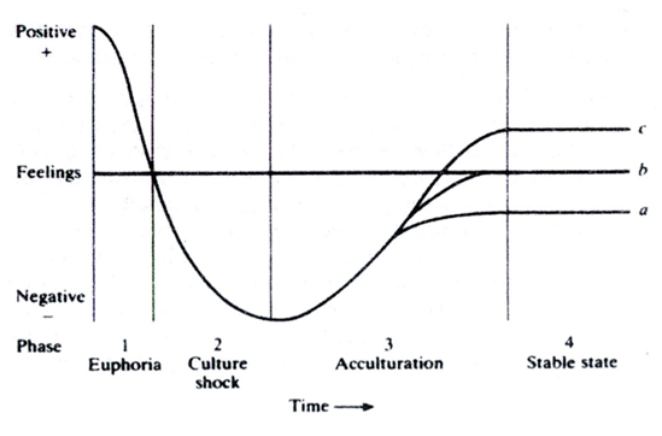

Key

a- continue to feel foreign

b- feel comfortable in both cultures.

c- be assimilated to the local culture more than the natives

Figure 1. The Acculturation Curve P385 $5^{[34]}$

Hofstedes' curve gives some insight into twins' changing experience through time: however, it does not account for the individual variations as pointed out by Adler and Gundersen (2008) [38]. Eriksen (2017) suggests that culture can also be used unconsciously to disguise personal values and limitations ${ }^{[39]}$. This may have occurred during this project when generalisations were made, for example in the case of individualist or collectivist culture that were then used to support value judgements regarding honesty or freedom of speech.

Trying to understand the group dynamics using Hofstede's five dimensions of cultural difference and the acculturation curve provides a basic understanding of what twins experienced, but its compartmentalisation is also dangerous because it is not the complete story. By equating culture with nationality, the complexity of national and cultural diversity is ignored [40]. Signorini et al. take Hofstede's approach to task for its inability to deal with 'the innate fuzziness of culture' and for its focus on differences instead of commonalities ${ }^{[41]}$. In line with this, our data reveal the flexible and dynamic nature of culture. Twins experience success, not by focussing on their differences, but by focussing on similarities and joint goals.

Two subthemes, that emerged under the theme cultural exchange during the analysis of the midterm and final focus groups, are well known phenomenon described in the business literature. The first subtheme, that centres on how the differences in culture afforded an opportunity for professional growth, is commonly described as a culture-gap and is defined as a cultural difference that can be bridged by people being prepared to work on this

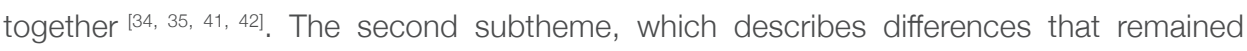

irritating and unresolved is described as cultural friction, defined as a cultural difference that cannot be bridged or can even cause conflict. The Financial Times Lexicon refers to cultural friction as 'the extent to which two or more entities, such as organisations, units, teams, group, and individuals, from different countries culturally resist, think or act in opposition' [44]. In addition to this, Sam (2010) shows that there is a correlation between how well people bridge a cultural gap and their ability to adapt in general ${ }^{[45]}$. It is therefore likely that the personal preparedness of twins to accept cultural differences also facilitated their professional growth ${ }^{[45]}$.

The complex dynamics of twinning are clearly reflected in the data. The time aspect, the cultural context, and the personal characteristics of twins are only some of the many interacting and continually changing factors, making the direction of cause and effect difficult to distinguish. For example, is cultural friction caused by a large culture-gap between twins or by the unpreparedness of twins to bridge this culture-gap? Does cultural friction hinder professional growth or does the lack of professional growth cause cultural friction? Our data expose the complex dynamics of twinning, but are not able to give us a clear answer to these questions. Being aware of the many ways culture shapes, and is used by participants in cross cultural interactions underscores these complex dynamics.

\section{CONCLUSION AND RECOMMENDATIONS}

After four years, the expectations of the twins were partially fulfilled with a noticeable difference between the Dutch twins who reported less, and Moroccan twins who reported more professional growth. This growth included their leadership capacity and agency to strengthening their midwifery association and thereby contribute positively to midwifery care. The data indicate that professional growth is hindered by cultural friction and may also be influenced by twins being either prepared or unprepared to bridge a culture gap. A focus on common (midwifery) goals, an adaptable personality and a reciprocal equitable environment facilitates the preparedness of twins to navigate the culture-gap. Added to this, the dynamics of twinning are impacted by a complex combination of personal values influenced by changing contexts and by twins confusing their personal values for cultural norms. All this said, twinning appears to be worthwhile.

We therefore recommend that initiators of twinning projects may optimise the potential professional growth of twins by being aware of the dynamics through time, matching twins from contexts with a graspable culture-gap, encouraging twins to work on common goals (in this case the midwifery products that contributed to maternal health) and taking into 
account the preparedness of twins to bridge a culture-gap during their recruitment and throughout the project.

The effect of the intense interaction between individual twins on their own professional growth and the overall complex dynamics of twinning is worth investigating further.

\section{CONFLICT OF INTEREST}

None.

\section{REFERENCES}

1. Kuruvilla, S., Bustreo, F., Kuo, T., Mishra, C. K., Taylor, K., Fogstad, H.,... \& Rasanathan, K. (2016). The Global strategy for women's, children's and adolescents' health (2016-2030): a roadmap based on evidence and country experience. Bulletin of the World Health Organization, 94(5), 398. doi: 10.2471/BLT.16.170431.

2. Coe, I. (2019). Feminism is for everybody. The Lancet, 393(10171), 493. https://doi.org/10.1016/501406736(19)30239-9.

3. Mwaniki, M. K. (2016). Quality in provision of maternity services: the missing link in health-care investments in LMICs? The Lancet Global Health, 4(11), e769-e770. http://dx.doi.org/10.1016/S2214-109X(16)30239-X

4. Miller, S., Abalos, E., Chamillard, M., Ciapponi, A., Colaci, D., Comandé, D.,... \& Manuelli, V. (2016). Beyond too little, too late and too much, too soon: a pathway towards evidence-based, respectful maternity care worldwide. The Lancet, 388(10056), 2176-2192. https://doi.org/10.1016/S0140-6736(16)31472-6.

5. Renfrew, M. J., Homer, C., Downe, S., McFadden, A., Muir, N., Prentice, T., \& ten Hoope-Bender, P. (2014). Midwifery: an executive summary for the Lancet's series. Lancet, 384(1), 8.

6. 22. ten Hoope-Bender, P., de Bernis, L., Campbell, J., Downe, S., Fauveau, V., Fogstad, H.,... \& Renfrew, M. J. (2014). Improvement of maternal and newborn health through midwifery. The Lancet, 384(9949), 1226-1235. http://dx.doi.org/10.1016/S0140-6736(14)60930-2.

7. Renfrew, M. J., McFadden, A., Bastos, M. H., Campbell, J., Channon, A. A., Cheung, N. F..... \& McCormick, F (2014). Midwifery and quality care: findings from a new evidence-informed framework for maternal and newborn care. The Lancet, 384(9948), 1129-1145. http://dx.doi.org/10.1016/ S0140-6736(14)60789-3.

8. World Health Organization (WHO).(2018). WHO recommendations on intrapartum care for a positive childbirth experience. World Health Organization. ISBN 978-92-4-155021-5.

9. Kennedy, H. P., Cheyney, M., Dahlen, H. G., Downe, S., Foureur, M. J., Homer, C. S.,... \& Soltani, H. (2018). Asking different questions: A call to action for research to improve the quality of care for every woman, every child. Birth, 45(3), 222-231. doi:10.1111/birt.12361.

10. White Ribbon Alliance. What Women Want. (2019). [cited 2020] Available from: https://static1.squarespace. com/static/5aa813dd3917ee6dd2a0e09e/t/5d1120ccdf7cbc0001b99c57/1561403606693/What-WomenWant_Global-Findings.pdf P45.

11. World Health Organization (WHO), International Confederation of Midwives(ICM), White Ribbon Alliance(WRA). (2016). Midwives voices, midwives realities. Findings from a global consultation on providing quality midwifery care. ISBN 9789241516112.

12. United Nations Population Fund (UNFPA). (2014). The State of the World's Midwifery, a universal pathway. A woman's right to health. Editor Bernis L.D. UNFPA, ICM, WHO: New York. ISBN: 978-0-89714-026-3 Available from: http://www.unfpa.org/sowmy.

13. Homer, C. S., Friberg, I. K., Dias, M. A. B., ten Hoope-Bender, P., Sandall, J., Speciale, A. M., \& Bartlett, L. A. (2014). The projected effect of scaling up midwifery. The Lancet, 384(9948), 1146-1157. http:// dx.doi. org/10.1016/S0140-6736(14)60790-X

14. Mosedale, S. (2014). Women's empowerment as a development goal: taking a feminist standpoint. Journal of 
International Development, 26(8), 1115-1125. https://doi.org/10.1002/iid.3050.

15. Filby, A., McConville, F., \& Portela, A. (2016). What prevents quality midwifery care? A systematic mapping of barriers in low and middle income countries from the provider perspective. PloS one, 11(5). doi: 10.1371/ journal.pone.0153391

16. United Nations Population Fund (UNFPA). (2019). The Maternal and Newborn Health Thematic Fund Case Studies on Strengthening Midwifery Services to Avert Maternal and Newborn Deaths p. 20. UNFPA: New York.

17. Van Lerberghe, W., Matthews, Z., Achadi, E., Ancona, C., Campbell, J., Channon, A.,... \& Koblinsky, M. (2014). Country experience with strengthening of health systems and deployment of midwives in countries with high maternal mortality. The Lancet, 384(9949), 1215-1225. http://dx.doi.org/10.1016/S0140-6736(14)60919-3.

18. World Health Organization (WHO), UNFPA, ICM, UNICEF. (2019). Strengthening quality midwifery education for Universal Health Coverage 2030: framework for action. ISBN 978-92-4-151584-9.

19. World Health Organization (WHO). (2018). Partnership Preparation Package. A practical document to implement twinning partnerships. WHO: Geneva. [cited 2020] Available from: https://apps.who.int/iris/bitstream/ handle/10665/273158/WHO-HIS-SDS-2018.13-eng.pdf.

20. Sandwell, R., Bonser, D., Hebert, E., Kilroy, K., Leshabari, S., Mwanga, F.,... \& Moritz, A. (2018). Stronger together: midwifery twinning between Tanzania and Canada. Globalization and health, 14(1), 1-10, p123. https://doi.org/10.1186/s12992-018-0442-x.

21. International Confederation of Midwives (ICM) twinning manual. (2014). Editor Moyo N. T. the Hague.

22. Chang, H. J. (2010). Bad Samaritans: The myth of free trade and the secret history of capitalism. Bloomsbury Publishing USA

23. Cadée, F., Nieuwenhuijze, M. J., Lagro-Janssen, A. L. M., \& De Vries, R. (2016). The state of the art of twinning, a concept analysis of twinning in healthcare. Globalization and health, 12(1), 66. doi: 10.1186/s12992-0160205-5.

24. Japanese Midwives Association. (2018). Midwives Reducing Obesity in Mongolia. Midwives For Life. [cited 2018]. Available from: http://www.m2025-weobservatory.org/midwives-reducing-obesity-in-mongolia.html.

25. Kemp, J., Bannon, E., Mwanja, M., Tebuseeke, D.(2018). Developing a national standard for midwifery mentorship in Uganda, International Journal of Health Governance, 23(1): p.81-94. https://doi.org/10.1108/ IJHG-09-2017-0051.

26. Ireland, J., Van Teijlingen, E., \& Kemp, J. (2015). Twinning in Nepal: the Royal College of Midwives UK and the Midwifery Society of Nepal working in partnership. Journal of Asian Midwives, 2(1), 26-33. http://ecommons. aku.edu/jam/vol2/iss 1/5/.

27. Cadée, F., Perdok, H., Sam, B., de Geus, M., \& Kweekel, L. (2013). 'Twin2twin'an innovative method of empowering midwives to strengthen their professional midwifery organisations. Midwifery, 29(10), 1145-1150. https://doi.org/10.1016/j.midw.2013.07.002

28. midwives4mothers charity. Twinning up North. Twinning project Iceland and the Netherlands. [cited 2019]. Available from: https://midwives4mothers.n//projecten/twinning-up-north/.

29. midwives4mothers charity. South - South Twinning. [cited 2019]. Available from: https://midwives4mothers.nl/ projecten/twinning-south-to-south/
30. Krueger, R. A. (2014). Focus groups: A practical guide for applied research. Sage publications. ISBN 978-14833-6524-4

31. Dedoose, Web application for managing, analysing, and presenting qualitative and mixed method research data Version 7.0.23. Los Angeles, CA: SocioCultural Research Consultants, LLC 2016. Available from: https://www. dedoose.com/.

32. Burla, L., Knierim, B., Barth, J., Liewald, K., Duetz, M., \& Abel, T. (2008). From text to codings: intercoder reliability assessment in qualitative content analysis. Nursing research, 57(2), 113-117. doi: 10.1097/01. NNR.0000313482.33917.7d

33. Tong, A., Sainsbury, P., \& Craig, J. (2007). Consolidated criteria for reporting qualitative research (COREQ): a 32-item checklist for interviews and focus groups. International journal for quality in health care, 19(6), 349-357. https://doi.org/10.1093/intqhe/mzm042.

34. Hofstede, G., Hofstede, G. J., \& Minkov, M. (2010). Cultures and Organizations: Software of the Mind McGrawHill Education. Interpretation Handbook and Standard. Distilling the Essence (2005).(ed. by Fiona Colquhoun), Department of Conservation, Wellington. ISBN 978-0-07-166418-9.

35. Meyer, E. (2016). The Culture Map (INTL ED): Decoding How People Think, Lead, and Get Things Done Across Cultures. Public Affairs. ISBN 978-1-61039-671-4.

36. Carbone, S., Wigle, J., Akseer, N., Barac, R., Barwick, M., \& Zlotkin, S. (2017). Perceived reciprocal value of health professionals' participation in global child health-related work. Globalization and health, 13(1), 27. https:// doi.org/10.1186/s12992-017-0250-8.

37. Cadée, F., Nieuwenhuijze, M. J., Lagro-Janssen, A. L., \& de Vries, R. (2018). From equity to power: Critical Success Factors for Twinning between midwives, a Delphi study. Journal of advanced nursing, 74(7), 15731582. https://doi.org/10.1111/jan.13560.

38. Adler, N. J., \& Gundersen, A. (2007). International dimensions of organizational behavior. Cengage Learning. ISBN 13:978-0-324-36074-5.

39. Eriksen, T.H. (2017) What is anthropology. Second edition ed, ed. Erikson. London: Pluto Press. ISBN-10: 0745399657

40. Baskerville, R. F. (2003). Hofstede never studied culture. Accounting, organizations and society, 28(1), 1-14. https://doi.org/10.1016/S0361-3682(01)00048-4.

41. Signorini, P., Wiesemes, R., \& Murphy, R. (2009). Developing alternative frameworks for exploring intercultural earning: a critique of Hofstede's cultural difference model. Teaching in Higher Education, 14(3), 253-264. https://doi.org/10.1080/13562510902898825

42. Jiang, H., Erickson, J. I., Ditomassi, M., \& Adams, J. M. (2012). Promoting a culture of international professional practice for nursing through a twinning relationship. JONA: The Journal of Nursing Administration, 42(2), 117122. doi: 10.1097/NNA.0b013e318243384e.

43. European Community (EC). (1999). TACIS City Twinning Programme. [cited 2015]. Available from: http:// ec.europa.eu/agriculture/rur/leader2/rural-en/euro/p8-1-5.htm.

44. Financial Times Lexicon. (May 2019). [cited 2020]. Available from http://markets.ft.com/research/Lexicon/ Term?term=cultural-friction. 
45. Sam, D. L., \& Berry, J. W. (2010). Acculturation: When individuals and groups of different cultural backgrounds meet. Perspectives on psychological science, 5(4), 472-481. https://doi.org/10.1177/1745691610373075. 


\section{ABSTRACT}

\section{BACKGROUND}

Twinning collaborations, where two groups - from educational institutions, hospitals or towns - work together cross-culturally on joint goals, are increasingly common worldwide. Pairing up individuals, so-called twin pairs, is thought to contribute to successful collaboration in twinning projects, but as yet, there is no empirical evidence or theory that offers insight into the value of the pair relationship for twinning.

To explore the contribution of one-to-one relationships between twins to twinning projects, as exemplified in projects between Dutch and Moroccan, and Dutch and Sierra Leone midwives.

\section{METHODS}

We conducted thirteen in-depth interviews with midwives from two twinning collaborations. Interviews were transcribed and analysed using an iterative, grounded theory process, yielding a theoretical understanding of one-to-one twinning relationships for twinning collaborations

\section{FINDINGS}

Participant comments fell into four substantive categories: 1) Being named a twin, 2) moving beyond culture to the personal level, 3) searching for common ground to engage, 4) going above and beyond the twinning collaboration. Their interplay demonstrates the value of twin pairs in paving the way for successful twinning.

\section{DISCUSSION}

A complex combination of contextual inequities, personality, and cultural differences affect the twin relationship. Trusting relationships promote effective collaboration; however, as 'trust' cannot be mandated, it must be built by coaching twins in personal flexibility and (cultural) communication.

\section{CONCLUSION}

By offering original insights into the ways twinning relationships are built, our research explores how twin pairs can enhance the success of twinning projects.

\section{KEYWORDS}

Twinning, midwives, trust, collaboration, culture, qualitative research.

\section{INTRODUCTION}

There is substantial evidence showing that when midwives work in an enabling environment, they are better able to support childbearing women and their babies realise their human right to quality sexual and reproductive healthcare ${ }^{[1-9]}$. Strong professional associations of midwives are an essential part of this enabling environment because they promote the development and implementation of quality midwifery care for women and their families, and stimulate midwives to speak up for midwifery with one voice ${ }^{[1,3]}$. Twinning has been identified as a means to strengthen midwife associations and to promote the professional growth of midwives ${ }^{[10]}$. Research shows the positive impact of twinning and underscores the importance of creating an evidence base for this type of collaboration ${ }^{[11-16]}$.

A growing number of educational institutions, hospitals, professional organisations, and towns are using twinning to strengthen each other through cross-cultural learning and the exchange of experience and practise ${ }^{[12,14,17-21]}$. The growing popularity of twinning has also captured the imagination of midwives, resulting in a rise in the number of twinning collaborations between associations of midwives globally. A membership survey by the International Confederation of Midwives (ICM) in 2019 found an increase in the number of midwife associations that want support from ICM to set up new twinning collaborations ${ }^{[22]}$.

Twinning collaborations can vary in the way they are organised, but the essence of this form of collaboration is captured in this recently published operational definition: a "crosscultural, reciprocal process where two groups of people work together to achieve joint goals' p1 [23]. Most twinning collaborations are between groups from twinned institutions who work together on joint goals. Research on these collaborations has focussed on the overall outcomes of this group process [17, 24-26]. Group twinning can struggle with issues of ownership due to power inequities and cultural and income differences. This may create subjectively constructed and normative group knowledge, which gets in the way of reciprocal learning and growth, important elements of successful twinning ${ }^{[21]}$.

In an effort to avoid this type of problem, a small, but growing, number of twinning collaborations has added individual twin pairing to the collaboration, as a way to encourage bilateral trust and personal commitment to twinning [19, 27-29]. This intervention originated spontaneously as an answer to issues of problem ownership during the Dutch-Sierra Leone twinning collaboration [27]. In our recent qualitative study exploring the group processes in twinning, twins reflected on the important role of twin pairs in bringing an extra personal dimension to understanding each other ${ }^{[15]}$. Our Delphi study consulting 33 midwife experts in twinning identified 25 critical success factors (CSF) for twinning, ten of which pertain directly 
to the importance of one-on-one interaction and relationship. Included among these ten are such things as being prepared to receive and give feedback, to trust and respect each other, and take into account each other's cultural norms ${ }^{[16]}$. A focus on personal interaction and conscious reflection is believed to stimulate dialogue between participants, resulting in positive engagement and the creation of trust, which, in turn, facilitates reciprocal learning [29]. To date, however, there have been no empirical studies of the effects of the one-to-one pairing in twinning collaborations.

In this study, we explore the value of one-to-one twin pairs in the context of two twinning collaborations between the midwife associations of 1) Sierra Leone and the Netherlands and 2) Morocco and the Netherlands. Better understanding of the role of twin pairs can contribute to the success of future twinning collaborations, amplifying the benefits of twinning, not only for midwifery associations, but for other international twinning projects as well.

\section{METHODS}

To gain an in-depth understanding of the value of twin pairs, we used semi-structured interviews supplemented by notes from discussions with members of the team that managed the twinning collaborations. Because there is no theory that explains the contribution of twin pairs to twinning collaborations, we used an inductive, grounded theory approach as described in our analysis.

\section{SETTINGS AND PARTICIPANTS}

Seven twin pairs $(N=14)$, were asked if they were willing to participate in individual in-depth interviews, conducted by the first and second authors. Four pairs $(\mathrm{N}=8)$ were selected from the twinning collaboration between the midwife associations of Sierra Leone and the Netherlands (2013-2016), a project that involved 50 midwives, or 25 pairs. Three pairs ( $N=6)$ were selected from the twinning collaboration between the midwife associations Morocco and the Netherlands (2014-2017), a collaboration involving 36 midwives, or 18 pairs. The indepth interviews in the Netherlands were done in April 2017 and June 2018, in Morocco in April 2017, and in Sierra Leone in February 2018. To obtain the broadest possible spectrum of insights, we used purposeful sampling, selecting twin pairs based on differences in the observed intensity of commitment to their twin relationships, as perceived by the twinning management team

Similar to the larger group of twins in both twinning collaborations, participants in the indepth interviews had been paired on the basis of their professional function or expertise: teachers to teachers, students to students, practising midwives to practising midwives, and managers/board members to managers/board members ${ }^{[27]}$. For the purpose of consistency, we will refer to these one-to-one paired midwives as twins or twin pairs throughout this article. The twins - as a group, as pairs, and individually - were supported as needed by a twinning management team in each collaboration. All participated in regular workshops about cross-cultural collaboration, leadership, midwifery practise and communication. Twin pairs took part in exchange visits to each other's countries and homes, attended the Triennial Congress of the International Confederation of Midwives (ICM) and presented their achievements in a final exhibition in their respective countries (15).

\section{DATA COLLECTION}

In-depth interviews were conducted using an interview guide built on the basis of observations from the twinning management team and the 25 critical success factors for twinning ${ }^{[16]}$. The experience of each interview was used as input to adapt the interview guide for the following interviews. All interviews were held face to face, audio-recorded and transcribed. The first and second author were present at the interviews.

In Morocco, the interviews were held either in French in the presence of a professional interpreter, or in English, depending on the language skills of the interviewee. The interpreter transcribed the French recordings to English. In the Netherlands, the in-depth interviews were held in Dutch, and the recordings were transcribed into Dutch. Relevant data from these interviews were translated into English for publication. In Sierra Leone, all interviews were held, and subsequently transcribed, in English. The transcriptions were sent to all twins for an accuracy check and optional additional comments. The quotes used here are drawn equally from all participants. Where necessary to ensure clarity, words were added to the quotes using square brackets [ ].

\section{ETHICAL CONSIDERATIONS}

During the initial invitation email, the confidentiality of participating midwives was assured. They were informed that their participation was voluntary and that a decision to not participate would have no consequence for their relationship with the research team or their midwife association. All participants were asked for their consent to use the data from their in-depth interviews for research purposes and were informed that the data would be securely stored at the research centre for midwifery science Maastricht, Zuyd University, the Netherlands. For reasons of confidentiality, the nationality of twins has not been added to illustrative quotes.

According to the act governing research involving human subjects in the Netherlands 
(WMO), formal, written ethical approval by a research ethics committee is required only for medical research where participants are subject to interventions or procedures, or are required to follow specific, research-related rules of behaviour ${ }^{[30]}$. Neither of these apply to this research. A self-assessment tool from the Medical Ethics Committee of Maastricht University, the Netherlands, confirmed that our study is exempt from formal medical ethical review [31]. This self-assessment tool takes into account physical, psychological, and economic harms as well as harms relating to privacy concerns.

\section{DATA ANALYSIS}

Following Glaser and Strauss's inductive approach, the first in-depth interview was analysed by the first author, resulting in an initial set of codes and categories ${ }^{[32]}$. These were then discussed and adjusted in conversation with the second author. These codes and categories were compared for overlap and changed and/or reassembled and subsequently applied to the analysis of the next in-depth interview. We then analysed two interviews from two other midwife associations to maximise our initial exploration of cultural difference. From that point on, the order of our analysis was by date of performance. The codes and categories that emerged were adapted several times during the analysis of the first seven interviews, after which saturation occurred. The remaining interviews were analysed to check for novel themes. No new categories were identified. Dedoose, an online programme for managing, analysing, and presenting qualitative and mixed method research data, was used to analyse our data ${ }^{[33]}$.

\section{RIGOUR AND REFLECTIVITY}

All authors were experienced in conducting qualitative research as well as familiar with midwifery and the concept of twinning. The first author compiled the interview guide, conducted the interviews, and lead the analysis of the transcriptions. The second author was present at all the in-depth interviews and provided an experienced and critical eye throughout the process. All authors gave feedback on the process and discrepancies were discussed until consensus was reached. We followed the Standards for Reporting Qualitative Research (SRQR) as a guideline for the reporting process ${ }^{[34]}$.

\section{FINDINGS}

Thirteen twins ( $N=13)$, out of the 14 twins initially approached, took part in the in-depth interviews. Of these twins, four were from Sierra Leone, three from Morocco and six from the Netherlands. We had an uneven number of participants because one twin from the Netherlands decided not to participate after her twin from Sierra Leone had already been interviewed. Even though the interview of this Dutch twin was not available, the interview of the midwife from Sierra Leone was considered valuable and was therefore included. Eight twins responded to the request to confirm the accuracy of their transcribed interview and one made additional comments that were incorporated into her interview.

\section{CATEGORIES}

Overall, our interviews and fieldnotes show that twinning was experienced as a worthwhile and, at the same time, complicated process due to continuous interaction of contextual, personal and cultural differences. Words like 'Intensive', 'unlearning', 'not understanding' and 'adapting' were common in the interviews. In our notes, we observed that twins regularly referred to the contextual differences with their twin, such as income, gender equity, education, legal status, socio-political issues, and hierarchy. Cultural differences were most often articulated with specific references to the way twins expressed emotions and opinions differently in public and in private situations. Twins regularly reflected on this: 'We are quick in expressing what is good or not good. They will not say that so openly, which is also nice' (twin 11).

Four categories emerged in our analysis: 1) the significance of being named a twin, 2) the need to move beyond culture to the personal level, 3) the search for common ground to engage, and 4) going above and beyond the twinning collaboration. Each category continuously interacts with the other categories and, taken together, form the basis for a theory of the contribution of twin pairs to (successful) twinning (Figure 1).

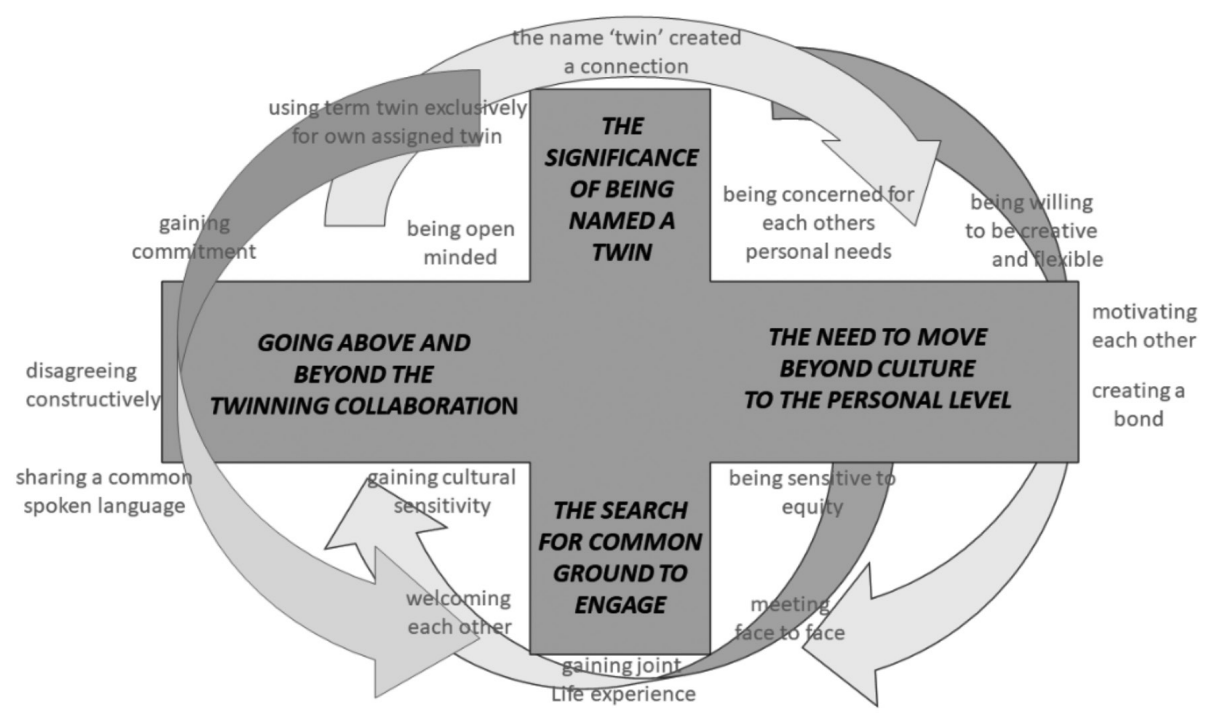

Figure 1. From categories to four substantive categories. 


\section{THE SIGNIFICANCE OF BEING NAMED A TWIN}

The idea of 'twins', two persons born from one mother in the same pregnancy who may be identical, generally appealed to our participants. The Sierra Leone twins spontaneously added 'sister' to the word twin and from then onwards the term 'twin sister' was used in most communications about the project and spontaneously adopted by most participants of the two twinning collaborations described in this study as well as new collaborations after the study was completed ${ }^{[13]}$.

Twins clearly voiced their special feeling for their assigned twin during the in-depth interviews. Twin 6 shared: "I more rely on my twin, because she was my twin sister and she was giving me more knowledge on the twinning project... I have [more] confidence in her than other... twins' and twin 9: 'It is lovely to have a mate within the project, that is my twin'. The impact that this word had on the identity of the participants as twins was remarkable; nearly all twins described a feeling of pride about being a twin and having a twin sister as expressed by twin 3: 'Because she was my twin I naturally had a special personal link with her'. It is noteworthy that the 'twin sister' was used solely for the assigned twin, even when new connections within the group were formed between twins: "I have twin $X$ as my real twin sister, I have twin $Y$ as a semi-twin, I have kind of adopted her" (twin 10). Twins struggled to put into words the special way they felt about having a twin, as illustrated by twin 11: 'it is hard to summarise, you kind of have a twin sister. But you don't share the same mother, yet that [twin sister] is what it is about, that you feel a real connection, which is different from being colleagues'.

Unlike relationships with colleagues generally, twins shared their private lives and their relationship grew through this personal dimension as described by twin 8: 'In the beginning it was worrying, I didn't know what to expect... but the first message from my twin reassured me... in these four years I made a friend... she's incredible we've been able to exchange everything, professional, family, ideas' and twin 7: 'every time we met, and all the things we experienced in our personal lives, it brought extra depth'. Some twin pairs were observed walking hand in hand at congresses.

On the down side, not all twin pairs connected well. Twin 1 said: '...the relationship hurt me...but when I look back I don't think of my sorrow but about the fun to build things together [with the others in the group]...' Some had different expectations of the length of the relationship. A clear example comes from the notes related to one in-depth interview where one twin expressed her pain, with tears in her eyes, of losing her twin with whom she had had intensive relationship during the collaboration, but who she never saw or heard of again afterwards.
The term twin also had an 'us and them' effect. The exclusivity of twin pairs caused a feeling of missing out by some other members of the midwife association outside the twinning collaboration who had not been paired up as twin. Twin 2 said: '...the moment you do one to one, they say...I'm not a friend of [twin X], I'm not close to her. The truth here is, how many of us [at the midwife association] related with all the twins? That was a hindrance'.

\section{THE NEED TO MOVE BEYOND CULTURE TO THE PERSONAL LEVEL}

One of the outstanding issues during the interviews was the interplay between twins' cultures and personalities, which included their views on equity and reciprocity. During the interviews, twins expressed the struggle of trying to adapt to being in a different culture whilst holding on to their own identity, assimilating new ideas while letting go of old norms and stereotypes. The individual inclusivity of their relationship with their twin appeared to overcome the problem of cultural exclusivity of the whole twinning group.

The different cultural contexts of twins are an integral part of twinning. Even though much attention was given to cross-cultural communication and cultural sensitivity during the workshops, the focus of twin pairs was primarily on each other as individuals as shared: 'if we want to keep the twin in our life, then we must ensure there is proper and regular communication' (twin 4). There were a few instances where twins described the others' culture as a hindrance to building their relationship: '... when a twin was very religious, and [her twin] had nothing [to do with religion] this was considered to be too difficult' (twin 5). Overall twin pairs supported the twinning process by lifting it to a personal, human level: 'the good thing about our relationship is the human aspect, it's our relationship itself...' (twin 8).

It became apparent to most twins that personal differences were at least as big as cultural differences: 'She [her twin] sure has to be open-minded, flexible... accept change, because we're different,... we should feel that effort to get closer... not [be] judgmental. So if we have these characteristics, we can have success, and [my twin] has all these things...' (twin 10). In navigating difficult issues - like equity, (dis)trust, expectations, disappointment, (de) motivation and temperament - twins learned the importance of their power-balance and how to support each other and avoid normative cultural judgements: '... when there really is an inequity of power or position... that does not work. One becomes the dependent child and the other the boss' (twin 3). This process of gaining insight in personal versus cultural issues resulted in personal growth for some twins described by twin 11: " experience that understanding this [having cultural judgements] affected me, yes, it increases my selfconfidence, my leadership capacity has really had a boost'. Face to face meetings and visiting their twin's home were mentioned by all twins as crucial for creating a basis of personal familiarity: '[it was] amazing to see how she lives and works, and since that time 
Most twins celebrated their differences, be it culture or personality, in an empowered and emancipated way. In separate interviews, each member of a twin pair independently disclosed the same story, each from their own perspective. Both laughed with joy and pride at each other's personal and cultural idiosyncrasies. This story illustrates the potential of twins to not only recognise and accept each other, but to celebrate their authentic differences: 'she was driving with [dyed] blue hair, in the exchange, my twin with blue hair!' (twin 12); and her twin (twin 11) recalled the same incident: 'We arrived in the cabrio [i.e. a convertible car], my twin with a headscarf. We were being watched...we discussed it and it was good and fun...'

\section{THE SEARCH FOR COMMON GROUND TO ENGAGE}

Twin pairs were responsible for creating their own sub-projects that related to the overall twinning collaboration goals set by both midwife associations. The negotiation process that preceded the choice of this sub-project created ownership amongst twin pairs: 'That project made us collide because we had the same concern, the same problem, so this concern, to be resolved or achieved, that concern motivated us' (twin 10). Twin pairs often chose their sub-projects within an area of common interest, which further strengthened their ownership. The midwifery sub-projects stimulated twins' enthusiasm to engage, as described by twin 6: "I decided to choose prevention of malaria in pregnancy, with my twinning sister...We wrote our projects and even made a cotton [wrapping cloth] so that people will know that it important for pregnant women to sleep under bed nets to prevent them from mosquito bites. This lappa cotton has even motivated other twin sisters'. In a few instances, there was no joint interest in a midwifery sub-project, a situation that nearly always went in conjunction with a mismatch between twins. In these cases, twins tended to find other twins to work with, as described by twin 9: 'the qualities of twin $X$ appealed more to me than my own twin ... because we could do better business [i.e. midwifery related work] together...'

Not sharing a common spoken or written language hindered finding common ground to engage. Twin 9 managed to overcome this hurdle 'I speak pretty good French, so for me that wasn't a handicap', but this was not the case for several twin pairs. Trying to communicate ideas and feelings in a second language or even through Google translate was a specific hindrance in the Morocco/Netherlands twinning collaboration. At the level of a shared way of thinking, twins made a special effort to keep the lines of communication open and constructive disagreement cultivated respect as mentioned by twin 1: 'Just looking at each other is enough now... through experience... we were open to each other...because we
In between face-to-face meetings, most twin pairs explored all manner of electronic communication including video messaging, WhatsApp, Skype, and email - and even letters by post - to continue the dialogue with the aim to progress their sub-project and relationship. Twin 9 illustrates that not all twins managed to find ways to bridge communication issues in between face-to-face meetings: '... we received no answers, ... whatever we posted or did, nothing happened. After our training in November we heard nothing unto April ... we were so disappointed...'. Twins pairs realised the importance of making the effort to understand each other: 'If both of them are not speaking with one voice ... believe me nothing good would happen ... a perfect twinning relationship is a good communication, sharing ideas together, coming out with initiatives that will be beneficial to both of us on the reduction of morbidity and mortality in my country' (twin 6).

\section{GOING ABOVE AND BEYOND THE TWINNING COLLABORATION}

Commitment by twins was expected when they were accepted initially into the twinning collaboration. This commitment was strengthened by a growing bond between them, as was relayed by twin 7: '...every time we met [our relationship] deepened with all the things we experienced in our personal lives'. As for many twins, twin 12 demonstrates how their bond grew through trust and respect for each other and developed to the level of a friendship: 'We start with professional twinning, and I think now we are friends'. All twins mentioned that their bond was strengthened by attending joint activities such as the International Confederation of Midwives (ICM) congress together: 'After the [ICM] congress in Durban was when I thought yes, this is the way I had expected it to be' (twin 1). The twin pairs observed and discussed each other as role models: 'I admired seeing twin $X$ and twin $Y$ [collaborate together]' (twin 13). Comparing themselves to other twins also highlighted challenges that hindered bonding between twin pairs. Twin 13 told us: 'I don't have a laptop...Even in my office I don't have a means of communication. So it's very difficult on my side to respond immediately... She [her twin] had wanted more. So things don't work that way for us, it was not smooth as the others.

Twin 7 shared how twins made a special effort to welcome each other to their respective homes, meeting family, friends and colleagues, and attending each other's professional work: 'it was a warm welcome... She had brought me lovely sweets and I had a book about birth for her. Yes, she liked that. It was a warm welcome', and twin 4 relayed: '... as if we've been together before...twin $X$ was so excited to meet me...so it was not like, oh, she is from Europe, I'm from Africa... You understand? Sometimes we have that inferiority complex. But we are able to accept each other'. Being able to focus on one twin made it possible to give 
that personal touch as is demonstrated by twin 8: 'the relationship was already there and the plus was the presence at her place, with her family...she took me to see her parents, I visited her sister also'. In the interviews, twins spoke in an animated way about how they were welcomed and how their twin had gone out of their way to make the visit a positive experience. Twin 12 spoke with fervour when she relayed her memory: 'it was my scarf, she told [her boyfriend]...to knock on the door before he went in. Yes, for me it's a big thing. That means to me respect'.

Twins often supported each other through times of difficulty or less motivation: 'When cultures are further apart it is better to go deeper and to attempt to connect, and that is great, really great... and a good motivator' (twin 3). As a result, activities unanticipated in the twinning plan were developed in addition to the overall collaboration goals, as illustrated by twin 5: 'I still don't understand why those people wanted to help... they had never met me...I just called them... amazing how it all turned out'. These additional extras gave a boost to the collaboration and other twins: 'I saw that twins that had a lot of contact had many projects, they even developed extra activities because of this personal contact' (twin 1).

Some twins did not make an extra effort for their twin, either because they had no shared interests or because their personalities did not match well. When this happened, a few twins disengaged from the twinning collaboration to different degrees, but most found others within the collaboration to engage with: '...there are different ways, I have my own way of looking at things... whatever you do, there is a downside. What my way is, having more people to work on somethings instead of the one to one' (twin 2).

\section{Toward a theory of twinning: The contribution of twin pairs for successful twinning} The four categories - 1) the significance of being named a twin, 2) the need to move beyond culture to the personal level, 3) the search for common ground to engage, and 4) going above and beyond the twinning collaboration - demonstrate a dynamic process that is described as an impactful experience by twins. Most twin pairs nurture in each other the motivation and willingness, or even the obligation, to persevere. In this way the four categories come together to make up an emergent theory, illuminating the ways twin pairs contribute to achieving their joint goals, and ultimately, to a successful twinning experience. Twin pairs pave the way for successful twinning (Figure 2).

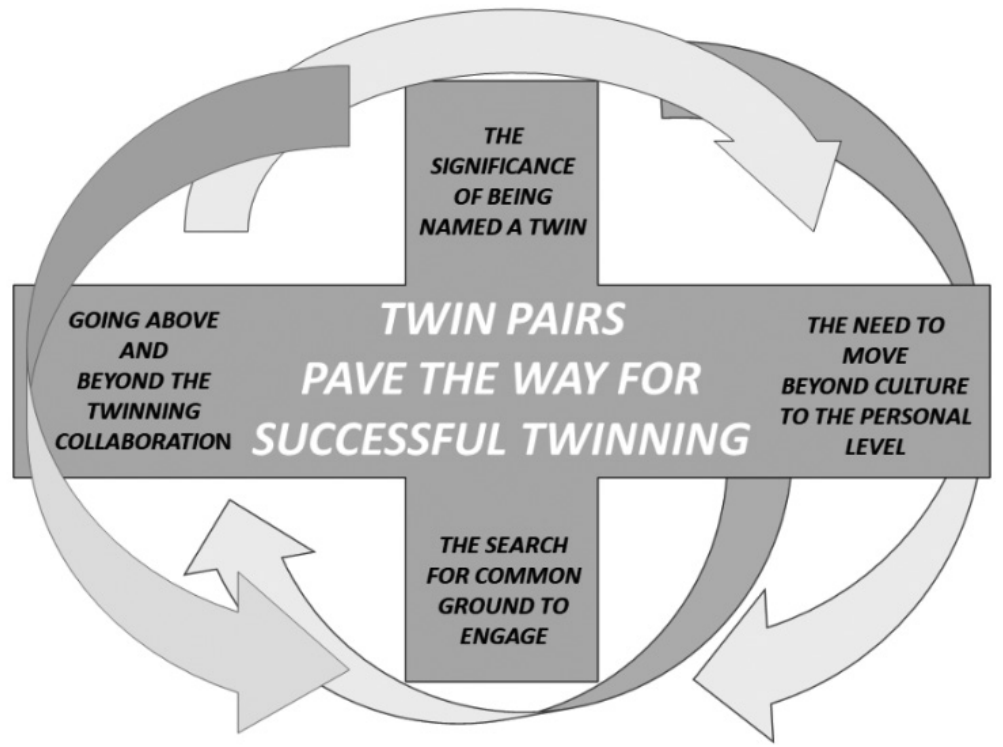

Figure 2. The emergent theory: Twin pairs pave the way for successful twinning.

\section{DISCUSSION}

Adding twin pairs to twinning collaborations appears to have had a positive impact on the twinning collaborations in this study. Paired twins reported both personal and professional growth and the twinning collaboration as a whole benefitted because the strength of the pair relationships safeguarded the group from human differences. However, even though twins paved the way for successful twinning, they did not act in isolation. Their activities, both together and separately, were interwoven with the whole group and the project team throughout the different aspects of the twinning collaboration.

\section{TWIN SISTERS, A NEW DIMENSION TO TWINNING}

Different cultural beliefs about twins may have complicated the personal identification with this term because twins are regarded differently in different cultures. In some cultures, twins are seen as a sign of virility of the father and in many, including Sierra Leone, being one of a twin is seen as a sign of good luck ${ }^{[35]}$. For some, becoming a twin meant that you are a twin for life, whereas for others this was not the case. This can explain the dismay some twins had after their relationship ended. Adding to these cultural beliefs surrounding twins, the twin pairs that took part in these two twinning collaborations were midwives who had varying professional experiences with caring for women in their midwifery practise. This too will have influenced the appeal of the term twin sister, which may not be experienced by 
other professionals involved in twinning in the same way. It is questionable if men would have adapted the term twin brother as readily as these midwives, all women, adopted twin sister. The degree to which one-to-one twinning would be embraced by men, or mixed-sex pairs remains as yet unexplored.

\section{GROUP CULTURE VERSUS INDIVIDUAL PERSONALITY}

It appears from the interview data that, at a personal level, twin pairs are more able to be nuanced than we found in our study of the group dynamics of the Moroccan-Dutch collaboration ${ }^{[15]}$. One to one, twins can no longer mask their personal views behind a cultural or professional façade and are therefore more likely to move away from cultural generalisations to see things in a contextual and personal perspective ${ }^{[36]}$.

The process of building trust between individuals, learning to value each other as autonomous human beings instead of seeing one another to be part of a generalised cultural group, has been well described ${ }^{[37]}$. Our findings from these two twinning collaborations reiterates that the hindrances experienced in building trust between twin groups, where normative values are more often at play, are more easily overcome between individual twin pairs ${ }^{[38]}$. Their individual bond supported twin pairs to make the twinning collaboration safer for human differences. Similar to Wilson (2017) we found that having sufficient professional similarities - by twins having similar midwifery interests - supported this bond [21]. Trust grows more easily between individuals when there is an understanding of the context, when we manage expectations, and suspend judgement by giving the benefit of the doubt ${ }^{[39,40]}$. Twins indicated that by visiting each other's homes and work, trust was more easily achieved with their twin than with the whole group at large. The individual relationship gave twins personal support and reassurance which helped them build their self-confidence to play their part within the twinning collaboration as a whole ${ }^{[41]}$. Learning to be more adaptable and moving beyond the group culture to the personal level added a personal dimension that gave the overall twinning collaboration a boost. It is, however, difficult to determine what came first: did twins gain adaptability by learning from the collaborations, or do twinning collaborations attract more adaptable people? Twin pairs bonded to differing degrees and their adaptability appeared to play an important part in the process. It is well known that flexible people tend to adapt more easily ${ }^{[42]}$. However, we do not claim that twins only build trust with their assigned twin exclusively. Trust clearly grew within the group as a whole during group gatherings such as their joint visit to midwifery conferences, exchange visits and workshops.

Even though the data indicate that twin pairs pave the way for successful twinning, twin pairs that did not have a good match did not pave the way, although they did not hinder others moving forward. A few twins that found the relationship with their own assigned twin too challenging, found other twins to relate to. The reasons given for the differences were mostly assigned to personality and rarely to culture.

\section{TWINNING IS COMPLEX}

When asked to describe the essence of the twin pair relationship, all twins struggled to put their ideas and feelings into words. Many twins pointed to their own relationship and a few pointed to other twin pairs as exemplary. Twins were able to say what their twin was not: she was not just a colleague, different from a friend, and not a real sister. Even though common midwifery interests and adaptability supported twins to build trusting relationships, predicting which twins pairs will or will not build a trusting relationships remains difficult.

Cause and effect are not linear in the twinning process; the interactions and interdependencies within the twinning group and between twin pairs occur in an ever-changing context. Twinning is described as a reciprocal process ${ }^{[23]}$. When we view this reciprocity as a complex adaptive process negotiated by twin pairs, we are better able to understand how twinning can be successful $[43,44]$. Complexity theory has its origins in physics and does not have the limitations of linear, reductionist thinking. Complexity theory can therefore deal better with the ambiguity and uncertainty expressed by twins, and with the unpredictability, creativity, and spontaneous self-organisation of twinning collaborations at large.

Complexity theory helps clarify why, in twinning, one size does not fit all. Every midwife association and twin pair steps into twinning differently - coming from different places and working in different contexts - and therefore successful results cannot be directly related to specific activities. The inevitability of unanticipated events underscores the unpredictable process of twinning and the need for adaptability ${ }^{[45]}$.

\section{STRENGTHS AND LIMITATIONS}

The strength of this study is that the data are extensive and rich as it was gathered from 13 participants from two twinning collaborations in low, middle, and high income countries from Europe, Northern and Sub-Sahara Africa. Saturation of the data was achieved after the analysis of the seven in-depth interviews, and the following six interviews affirmed the categories that emerged. This adds to the reliability and global applicability of the findings. The data were gathered between one and four years after finalisation of the twinning collaborations providing a long term perspective of participant views - and not just a positive "afterglow" that might be found immediately after the collaboration was completed. All authors have extensive backgrounds in qualitative research. The first author was deeply immersed in managing twinning collaborations, and all other authors have gained a substantial familiarity with the concept of twinning 
Our study also has limitations. Despite the fact that purposeful sampling - based on differences in the intensity of commitment to their twin relationships - was used to generate a balanced representation of the range of twin pair collaborations, the sampling was solely based on the impressions of the twinning management team. It is noteworthy that twin pairs who appeared to have overtly challenging relationships or little contact did not describe themselves as such. Even though we guaranteed confidentiality and made an effort to create an open constructive atmosphere during the in-depth interviews, it is still possible that twins' familiarity with the first author as well as cultural and personal differences in sharing these negative aspects of their experiences may have hindered them from expressing themselves freely or choosing to frame it positively as a learning experience. An argument can be made that the double role of twinning manager and researcher/first author could be problematic. However, qualitative methods in general, and grounded theory more specifically, call for intimate familiarity with the topic under study ${ }^{[32,46]}$. The position of the first author allowed that familiarity and the inclusion of two experienced researchers who were not involved in the twinning collaboration allowed a more objective view of the data. Finally, language issues meant that a professional interpreter was necessary for some of the in-depth interviews. Some nuance may have been lost during translation.

\section{CONCLUSION AND RECOMMENDATIONS}

Twin pairs relate positively to being named a twin, move beyond culture to the personal level, search for common ground to engage, and go above and beyond the twinning collaboration. This opened opportunities for twin pairs to pave the way for successful twinning. The dynamics of twinning collaborations are complex because of the interplay of personal, professional, group, organisational, and cultural processes within an everchanging context. The relatively new development of adding twin pairs gives an extra dimension to twinning, yet does not appear to complicate twinning further. Instead, twins help to navigate this complexity contributing to the joint goals of the project while bringing along additional unexpected positive results.

Bonding between twin pairs is enabled by twins building trusting relationships. This is facilitated by the adaptability of each twin as well as by issues such as meeting face to face, sharing a common language, making history together through joint activities in the private and midwifery sphere, and having shared interests. Clearly, not all twins built strong relationships, but in our study twins with relational challenges did not have a negative impact on the twinning collaboration as a whole.
It is tempting to think that there is a right way to set up twinning collaborations. However, the one size fits all idea is an illusion and set up to fail. The strength of twinning is that its appearance becomes apparent through a unique process that adapts itself to the reality of different contexts. This was reiterated by twin pairs themselves who were unable to pinpoint the essence of their relationship except for pointing out what it was not.

With the growing demand for twinning by midwife associations globally, we recommend the pairing of twins in twinning collaborations. Coaching twins personally and professionally, without coercion, can support them in building trusting relationships, crucial for bonding. Sharing a common spoken and written language, while not always possible, will reduce unnecessary communication complications. Pairing twins can also benefit other forms of collaborations such as exchanges, working visits, and partnerships.

\section{ACKNOWLEDGMENTS}

We would like to acknowledge all the midwives who took part in this study.

\section{AUTHOR STATEMENTS}

All authors have contributed substantially to the study conception and design, data acquisition, analysis and interpretation. All authors have contributed to the drafting of the article for intellectual content. All authors agree to be accountable for all aspects of the work related to the accuracy or integrity of any part of the work. All authors approved of the final version.

Authors' contributions:

\begin{tabular}{ll}
\hline Criteria & Author Initial \\
\hline The conception and design of the study, acquisition of data, analysis and interpretation of data & All authors \\
Drafting the article or revising it critically for important intellectual content & All authors \\
Final approval of the version to be submitted & All authors
\end{tabular}

\section{FUNDING}

This study received funding from the Stichting Bijzondere Voorzieningen Moederschapszorg (SBVM) in Heerlen, the Netherlands (Charity Special Provisions Maternity-care) to enable the first author to dedicate time to writing this article. The funders had no involvement in the study design, in the collection, analysis and interpretation of data, in the writing of the report and in the decision to submit the article for publication. No other funding was received from funding agencies in the public, commercial, or not-for-profit sector.

\section{CONFLICT OF INTEREST}

No conflict of interest has been declared by the authors. 


\section{REFERENCES}

1. United Nations Population Fund (UNFPA). (2014). The State of the World's Midwifery, a universal pathway. A woman's right to health. Editor Bernis L.D. UNFPA, ICM, WHO: New York. ISBN: 978-0-89714-026-3 Available from: http://www.unfpa.org/sowmy.

2. World Health Organization (WHO). (2018). WHO recommendations on intrapartum care for a positive childbirth experience. World Health Organization. ISBN 978-92-4-155021-5.

3. World Health Organization (WHO), International Confederation of Midwives (ICM), White Ribbon Alliance (WRA). (2016). Midwives voices, midwives realities. Findings from a global consultation on providing quality midwifery care. ISBN 9789241516112

4. World Health Organization (WHO). (2016). Global strategy for women's, children's and adolescents' health (2016-2030). [cited 2020] Available from: https://www.who.int/life-course/partners/global-strategy/globalstrategy-key-statistics-03-11-2017.pdf.

5. ten Hoope-Bender P, de Bernis L, Campbell J, Downe S, Fauveau V, Fogstad H, et al. Improvement of maternal and newborn health through midwifery. The Lancet. 2014;384(9949):1226-35. doi: 10.1016/s01406736(14)60930-2.

6. Renfrew, M. J., McFadden, A., Bastos, M. H., Campbell, J., Channon, A. A., Cheung, N. F., ... \& McCormick, F. (2014). Midwifery and quality care: findings from a new evidence-informed framework for maternal and newborn care. The Lancet, 384(9948), 1129-1145. http://dx.doi.org/10.1016/ S0140-6736(14)60789-3.

7. Mwaniki, M. K. (2016). Quality in provision of maternity services: the missing link in health-care investments in LMICs? The Lancet Global Health, 4(11), e769-e770. http://dx.doi.org/10.1016/S2214-109X(16)30239-X.

8. Renfrew, M. J., Ateva, E., Dennis-Antwi, J. A., Davis, D., Dixon, L., Johnson, P.,... \& McFadden, A. (2019) Midwifery is a vital solution-What is holding back global progress?. Birth (Berkeley, Calif.), 46(3), 396. doi: 10.1111/birt.12442

9. Kennedy, H. P., Cheyney, M., Dahlen, H. G., Downe, S., Foureur, M. J., Homer, C. S.,... \& Soltani, H. (2018). Asking different questions: A call to action for research to improve the quality of care for every woman, every child. Birth, 45(3), 222-231. doi:10.1111/birt.12361.

10. International Confederation of Midwives (ICM) twinning manual. (2014). Editor Moyo N. T. the Hague.

11. Japanese Midwives Association. (2018). Midwives Reducing Obesity in Mongolia. Midwives For Life. [cited 2018]. Available from: http://www.m2025-weobservatory.org/midwives-reducing-obesity-in-mongolia.htm.

12. Sandwell, R., Bonser, D., Hebert, E., Kilroy, K., Leshabari, S., Mwanga, F.,... \& Moritz, A. (2018). Stronger together: midwifery twinning between Tanzania and Canada. Globalization and health, 14(1), 1-10. doi: 10.1186/ s12992-018-0442-x.

13. midwives4mothers charity.(2020). Twinning up North. Twinning project Iceland and the Netherlands. [cited 2019]. Available from: https://midwives4mothers.nl/projecten/twinning-up-north/

14. Ireland, J., Van Teijlingen, E., \& Kemp, J. (2015). Twinning in Nepal: the Royal College of Midwives UK and the Midwifery Society of Nepal working in partnership. Journal of Asian Midwives, 2(1), 26-33. http://ecommons. aku.edu/jam/vol2/iss $1 / 5$.
15. Cadée, F., Nieuwenhuijze, M. J., Lagro-Janssen, A. L., \& de Vries, R. (2020). Embrace the Complex Dynamics of Twinning! SAGE Open (in review, minor changes).

16. Cadée, F., Nieuwenhuiize, M. J., Lagro-Janssen, A. L., \& de Vries, R. (2018). From equity to power: Critical Success Factors for Twinning between midwives, a Delphi study. Journal of advanced nursing, 74(7), 15731582. https://doi.org/10.1111/jan.13560

17. Weyreter, M. (2003). Germany and the town twinning movement. Contemporary Review, 282, 37-43.

18. University of Wisconcin-Madison. (2009). Ethiopia Global health Twinning. A Global Health Partnership between the University of Wisconsin-Madison, Addis Ababa University: University of Wisconcin-Madison. [cited 2019]. Avaliable from: https://www.surgery.wisc.edu/international-collaborations/ethiopia-global-health-twinningpartnerships/

19. Royal College of Midwives (RCM). (2015). Global midwifery twinning project. [cited 2020] Available from: https:// www.rcm.org.uk/promoting/global/projects/global-midwifery-twinning-project/.

20. Ndenga E, Uwizeye G, Thomson DR, Uwitonze E, Mubiligi J, Hedt-Gauthier BL. (2016). Assessing the twinning model in the Rwandan human resources for health programme: goal setting, satisfaction and perceived skill transfer. Glob Health12. doi: 10.1186/s12992-016-0141-4.

21. Wilson, A. (2017). International Twinning: The Foundation for Organizational Development and Program Growth. In Toronto: Conference Presentation presented at: International Confederation of Midwives Conference.

22. International Confederation of Midwives (ICM). (2019). Membership Survey. (Unpublished work). ICM, the Hague.

23. Cadée, F., Nieuwenhuijze, M. J., Lagro-Janssen, A. L. M., \& De Vries, R. (2016). The state of the art of twinning, a concept analysis of twinning in healthcare. Globalization and health, 12(1), 66. doi: 10.1186/s12992-0160205-5.

24. European Community (EC). (1999) TACIS City Twinning Programme. [cited 2015]. Available from: http:// ec.europa.eu/agriculture/rur/leader2/rural-en/euro/p8-1-5.htm.

25. Cooper, L. (1984). The twinning of institutions: its use as a technical assistance delivery system. The World Bank. [cited 2020]. Available from: http://agris.fao.org/agris-search/search.do?recordlD=US2012400749.

26. World Health Organization (WHO). (2001). Guidelines for city twinning. WHO Regional office Europe. [cited 2020]. Avaliable from: https://apps.who.int/iris/bitstream/handle/10665/107370/E74547. pdf? sequence=1.

27. Cadée, F., Perdok, H., Sam, B., de Geus, M., \& Kweekel, L. (2013). Twin2twin an innovative method of empowering midwives to strengthen their professional midwifery organisations. Midwifery, 29(10), 1145-1150. https://doi.org/10.1016/j.midw.2013.07.002

28. Kemp, J., Shaw, E., \& Musoke, M. G. (2018). Developing a model of midwifery mentorship for Uganda: the MOMENTUM project 2015-2017. Midwifery, 59, 127-129. doi: 10.1016/j.midw.2018.01.013.

29. Wilson, G., \& Johnson, H. (2007). Knowledge, learning and practice in North-South practitionerto-practitioner municipal partnerships. Local government studies, 33(2), 253-269. https://doi. org/10.1080/03003930701200544

30. Central Committee on Research involving Human Subjects the Netherlands (CNMO) (2019). [cited 2019]. Available from: https://english.ccmo.n/linvestigators/legal-framework-for-medical-scientific-research/yourresearch-is-it-subject-to-the-wmo-or-not. 
31. University of Maastricht. (2019). Ethics Review Committee Health, Medicine and Life Sciences. Website: Maastricht University, the Netherlands. [cited 2019]. Available from: https://www.maastrichtuniversity.n//aboutum/faculties/health-medicine-and-life-sciences/research/ethics-review-committee-health.

32. Levy, V. (1999). Protective steering: a grounded theory study of the processes by which midwives facilitate informed choices during pregnancy. Journal of advanced nursing, 29(1), 104-112. doi:10.1046/j.13652648.1999.00867.x.

33. Dedoose. (2016). Web application for managing, analyzing, and presenting qualitative and mixed method research data Version 7.0.23. Los Angeles, CA: SocioCultural Research Consultants, LLC. Available from: www. dedoose.com.

34. Network Standards for reporting qualitative research Oxford, UK. (2019). Centre for Statistics in Medicine (CSM), NDORMS, University of Oxford. [cited 2019]. Available from: http://www.equator-network.org/reportingguidelines/srar/.

35. Hide J. (2017). Twin traditions in various cultures Medium. [cited 2019]. Available from: https://medium.com/@ info_71574/twin-traditions-in-various-cultures-fd626a3ff847.

36. Eriksen, T.H. (2017). What is anthropology. Second edition ed, ed. Erikson. London: Pluto Press. ISBN-10: 0745399657

37. Kottak, C. P. (2011). Anthropology: Appreciating human diversity. McGraw-Hill.New York.

38. Kugler, T., Bornstein, G., Kocher, M. G., \& Sutter, M. (2007). Trust between individuals and groups: Groups are less trusting than individuals but just as trustworthy. Journal of Economic psychology, 28(6), 646-657. https:// doi.org/10.1016/j.joep.2006.12.003.

39. Möllering, G. (2001). The nature of trust: From Georg Simmel to a theory of expectation, interpretation and suspension. Sociology, 35(2), 403-420. https://doi.org/10.1017/S0038038501000190

40. Vangen, S., \& Huxham, C. (2003). Nurturing collaborative relations: Building trust in interorganizational collaboration. The Journal of Applied Behavioral Science, 39(1), 5-31. https://doi.org/10.1177/0021886303 039001001.

41. Kweekel, L., Gerrits, T., Rijnders, M., \& Brown, P. (2017). The role of trust in centeringpregnancy: building interpersonal trust relationships in group-based prenatal care in The Netherlands. Birth, 44(1), 41-47. https:// doi.org/10.1111/birt.12260

42. Sam, D. L., \& Berry, J. W. (2010). Acculturation: When individuals and groups of different cultural backgrounds meet. Perspectives on psychological science, 5(4), 472-481. doi: 10.1177/1745691610373075.

43. Glouberman, S., \& Zimmerman, B. (2002). Complicated and complex systems: what would successful reform of Medicare look like?. Romanow Papers, 2, 21-53. Toronto Canada. ISBN 0-8020-8616-7.

44. Cilliers, P. (2002). Complexity and postmodernism: Understanding complex systems. Routledge. https://doi. org/10.4324/9780203012253

45. Ramaswamy, R., Reed, J., Livesley, N., Boguslavsky, V., Garcia-Elorrio, E., Sax, S.,... \& Parry, G. (2018). Unpacking the black box of improvement. International Journal for Quality in Health Care, 30(suppl_1), 15-19. https://doi.org/10.1093/intqhe/mzy009.

46. Pawluch, D., \& Neiterman, E. (2010). What is grounded theory and where does it come from. The SAGE handbook of qualitative methods in health research, 174-192. ISBN 978-1-84787-292-0. 


\section{INTRODUCTION}

In this thesis we provide new and important insights into how twinning - a specific form of collaboration - works in healthcare with special reference to twinning collaborations between midwife associations. In this concluding chapter, we reflect on the main findings of our study using the lens of complexity theory, a useful tool for examining the reality of twinning in the field - that is, how twinning actually works. We also consider the limitations and strengths of the thesis and provide recommendations for future research and for practice.

\section{SUMMARY OF THE FINDINGS}

Widely touted, but rarely studied, twinning is offered as a way to strengthen both professionals and the organisations that support their work ${ }^{[1]}$. Our research offers one of the first systematic examinations of the twinning process. This thesis opened with an exploration of the general characteristics of twinning in healthcare. Because twinning is not well defined, we started our research with a concept analysis, allowing us to generate a new operational definition: 'Twinning is a cross-cultural, reciprocal process where two groups of people work together to achieve joint goals'. We next consulted experts to identify the ingredients necessary to make twinning successful. Using a Delphi process, we distilled twenty-five critical success factors, the majority of which focused on equity. We then looked at the factors that facilitate and hinder the professional growth of midwives who participate in twinning, using data collected from those who participated in twinning collaborations between the midwife associations of Morocco and the Netherlands. We found that cultural differences can both hinder and facilitate professional growth, depending on one's personal preparedness to bridge cultural differences. In our final study, we examined the role of the one-to-one twin pair relationship in the twinning process. The data for this study were drawn from two twinning collaborations: one between midwives from the associations of Sierra Leone and the Netherlands and another between the midwife associations of Morocco and the Netherlands. We found that in the midst of the complex dynamics of twinning, twin pairs can pave the way to success. Together, these four studies give a broad view of important aspects of twinning collaborations. Overall, we found that twinning enables midwives to develop the agency needed to play a proactive part in organising and delivering quality midwifery care.

Others who wish to use twinning as a way to develop professional expertise and agency can learn from the work presented here. However, in reflecting on what we learned in our research, it became clear that successful twinning requires an understanding of the complexity of the process and not just a step-by-step guide on "How to twin". In order to unpack the complexities of twinning, we look at our findings in light of complexity theory.

\section{TWINNING IS A COMPLEX PROCESS}

There can be a tendency to look at twinning as a linear process in which certain activities or interventions result in favourable outcomes. During the five-year process in which the four studies described in this thesis were carried out, it became increasingly apparent that twinning is not an intervention that can be introduced in a linear, straightforward fashion. It is, instead, a complex and dynamic process. This complexity can be found in its unpredictability, non-linearity, and its continuously changing, yet reoccurring patterns, or feedback loops that appear at different levels. These characteristics require special skills - including the ability to understand the specific context and an openness to adapt to unexpected change - on the part of those who wish to implement twinning projects [2-4]. Familiarity with complexity theory can help would-be implementers of twinning to expect and prepare for the unexpected.

Complexity theory has its origin in physics, where it emerged as a way to understand nonlinear processes such as the weather ${ }^{\left[{ }^{[}\right]}$. Applying complexity theory to social processes addresses the limitations of linear, reductionist thinking, where a specific intervention results in a clear and anticipated result. Linear thinking cannot explain unexpected outcomes of interactive, creative processes between agents that appear to be unpredictable and spontaneously self-organised. In complexity theory, these agents are often people who have the capacity to exchange information with their environment and then adjust their behaviour accordingly. A diversity of agents, such as seen in twinning, increases the chances of changed patterns of behaviour ${ }^{[4]}$.

Our four studies demonstrate that twinning is made up of interconnected patterns and interactions amongst agents - including twins - within their changing context as individuals and groups ${ }^{[3]}$. A complexity theory lens helps us to recognize these interdependencies as well as the dynamics at play ${ }^{[2]}$. A focus on the process and patterns of the day-today activities of twinning - looking at the processes from a distance in its ever-changing context of past, present, and future - offers important insights into how twinning works ${ }^{[2}$, 4, 6]. This perspective requires asking different, non-linear, questions such as how one can learn from various disruptions and adapt the implementation accordingly. Reflecting on how these adaptations come forward in project evaluations, we can explore how contextual issues influence the process of twinning and better understand adaptation as a unique, 
but generalisable concept. Whilst we cannot specify exactly how a twinning team must adapt, we can point to the need to allow for the influence of specific contexts and general directions. By giving credit to the complexity of twinning, in which every situation requires a unique approach, we can strengthen its positive impact ${ }^{[7]}$.

Nieuwenhuijze et al., developed a taxonomy, or classification system, of the complexity theory in healthcare, identifying eleven main components. These components are also applicable in the context of maternity care, illustrating the dynamic processes at play (Table 1.) ${ }^{[5]}$.

Table 1. A taxonomy for complexity theory in healthcare, adapted from Nieuwenhuijze (2015) P6 ${ }^{[5]}$.

1. Simple rules: Shared, internalized principles or values that can be hard to determine explicitly and are broad enough to provide space for creativity.

2. Emergence: Systems that develop (emerge) and carry on adapting over time in response to their context and to internal and external challenges. Diversity of agents enhances interaction and enhances the development of new and richer patterns.

3. Interconnection: The system thrives on a dynamic range of agents (people) who interact together through interdependent relationships and who are also connected to various other systems with free flow of information. Open boundaries: The boundaries of the system are vaque and cannot be separated from the environment. Agents, information, and ideas move between systems and are part of other systems simultaneously.

5. Self-Organisation: Agents can adapt spontaneously to different challenges without hierarchical regulation.

6. Feedback loops: Interacting agents cause both positive and negative effects on each other and the context which affects their further behaviour.

7. Initial conditions: The history, background, or context of a system shapes the way we will look at the present

8. Non-linearity: Small changes can have large, non-proportional effects that are directly evident. The effect can S

9. Unpredictability: Random, unplanned occurrences appear and have an unpredictable effect. Accurate,

10. Co-evolution: A complex system co-evolves with its environment, because it is embedded in other systems. Mutual transformation is initiated by continuous change in the environment requiring new responses and

11. Attractors: Eventually, the system will achieve balance. This new situation is likely to be temporary as the system will adapt again when changes take place.

Each of these eleven components can be found in the twinning collaborations studied for this thesis. In an effort to allow this taxonomy to be used by those implementing twinning projects, we have organised the eleven components into three categories, framing them as considerations for those who wish to set up a twinning collaboration:

1. Pay attention to context.

2. Mind the process.

3. Be prepared for the unexpected.

Using this frame to think about the complexities that emerged in our research and the dayto-day management of the twinning collaborations studied helps us gain a broader view of the interdependent relationships between actions, interactions, and actors as a part of a dynamic complex process ${ }^{[8]}$

\section{PAY ATTENTION TO CONTEXT}

As our research progressed, it became clear that, even with the new operational definition described in chapter two and the development of 25 critical success factors presented in chapter three, it is unlikely that one standardised method will work for twinning. Worldwide, midwife associations are at different stages of development ${ }^{\left[{ }^{9}\right]}$. The education, regulation, and scope of midwifery is different in every country and continuously evolving ${ }^{[10]}$. Also, the position of midwives varies enormously and generally reflects the position of women in that same society ${ }^{[11,12]}$. Any attempt to twin two associations of midwives must take into account these contextualities. These initial conditions also determine the context because, even though the twinning path might not be linear, they give a sense of direction [13]. Two elements of complexity theory - initial conditions and simple rules - call for the need to pay attention to the contexts in which twinning operates (Table 2).

Table 2. Two context elements: From a taxonomy for complexity theory in healthcare (adapted from ${ }^{(5)}$ ).

\section{Initial con \\ and future.}

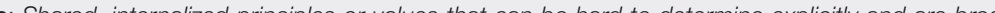
enough to provide space for creativity.

We see these two elements in our work. In our first study, an exploration of the general characteristics of twinning in healthcare, we noted that the initial conditions of each of the twinning collaborations included in the concept analysis were different ${ }^{[14]}$. The idea of twinning itself begins with initial conditions that differ from other, more classic forms of international aid, where, for the most part, the rich give to the poor. Twinning includes reciprocity as a core value, a feature of the process that is reiterated in the strong focus on equity found in the 25 critical success factors (Chapter 3). The "simple rules" of twinning - reciprocity and equity are broad and allow twins, as individuals, or in pairs or groups, to shape twinning according to their own personal initial conditions. We found that the personal backgrounds of twins, including their education, living conditions, and culture, influence the way twins assimilate to their role as twins. Additional simple rules that influenced the projects described here include a preparedness to bridge culture gaps (Chapter 4) which influences the variety of twins' strategies for paving the way to successful twinning (Chapter 5).

Understanding twinning as a complex dynamic system does not make twinning chaotic, as long as there is a clear joint commitment to the simple rules for twins. For midwives, 
these globally-shared simple rules include the shared philosophy of practice and a deep motivation to contribute towards quality sexual and reproductive healthcare and rights for women. For specific twinning collaborations, the attractors also include jointly agreed-upon goals set at the onset of twinning ${ }^{[5]}$.

\section{MIND THE PROCESS}

When we talk of the complexity of twinning, we do not mean that all aspects of twinning are complex. The combination of our four studies in this thesis have shown that twinning also encompasses simple and complicated issues. Simple issues, which generally need a basic technique that, once mastered, results in high assurance of success, includes things like the logistics of booking flights and arranging accommodation for 20 midwives. Complicated issues are more difficult but are still reducible to a series of steps. For example, it is a complicated task to prepare materials for a workshop on cultural sensitivity for two diverse groups, including the need to study and understand the role of culture in group dynamics and presentation strategies. There is a tendency to treat twinning as a machine with both simple and complicated elements that can be analysed, taken apart, and reassembled to allow for success ${ }^{[2,6]}$. However, twinning is complex and not reducible to a combination of plannable simple or complicated elements ${ }^{[15]}$. Complex issues require an understanding of changeable processes and flexibility to adapt ${ }^{[15]}$. The following elements of complexity theory give additional insight into twinning: interconnection, open boundaries, emergence, and co-evolution and attractors (Table 3).

Table 3. Five process elements: From a taxonomy for complexity theory in healthcare (adapted from ${ }^{(5)}$ ).

Interconnection: The system thrives on a dynamic range of agents (people) who interact together through interdependent relationships and who are also connected to various other systems with free flow of information Open boundaries: The boundaries of the system are vague and cannot be separated from the environment. Agents, information and ideas move between systems and are part of other systems simultaneously

Emergence: Systems that develop (emerge) and carry on adapting over time in response to their context and to internal and extent chalenges. Divesity of agents en aches interaction and enhances the development of new and richer petterns.

Co-evoluter system co-evolves with its environment, because it is embedded in other systems. initiated by continuous change in the environment requiring new responses and

a thentually, the system will settle down in a new balance. This new situation is likely to be temporary as the system will adapt again when changes take place.

The existence of the 25 critical success factors (CSF) for twinning (Chapter 3) may imply that implementation of the complete set of CSFs will guarantee success. This is an illusion. During twinning, the CSFs cannot be separated from the context, including the people. In our Delphi study we found, for example, that mutual commitment by twins to each other and to the overall twinning collaboration was considered critical to success. But in reality, as we learned in our projects, the way this commitment was interpreted by twins changed, because of having to adapt to the changes in depth and frequency of contact. Twins in our projects stayed together for several days, sharing private and work-related activities, and then they would not see each other for a year with only intermittent access to the internet. Some twins carried on with their agreed activities by themselves, and did not always communicate the progress made. Other twins became engrossed in the routine of daily midwifery and domestic work and suspended their twinning activities until it came close to the next moment of face-to-face contact.

Changing contexts, such as the Ebola crisis in Sierra Leone and the growing global tensions between ethnic and religious groups, were intertwined with both twinning collaborations given the open boundaries. As the context transformed, twins adapted and readapted in a variety of ways. This explains why a state of balance (attractor) was hard to achieve and wasn't always achieved. This process of adapting to changing circumstances reflects another of the critical success factors - "goals are adapted if circumstances change" (CSF 18) $P 8^{[16]}$. Communication issues such as language barriers as well as differences in access to, and use of, electronic means of communication like email, Skype, and WhatsApp, created the need to find new ways to work together. The 25 critical success factors are better seen as stepping-stones that enable the process to develop in its own unique way.

\section{BE PREPARED FOR THE UNEXPECTED}

Complexity theory takes into account the many elements of surprise that emerge in social processes. The twinning process was often unpredictable; understanding the dynamics at play was important to the success of the collaboration. Not all of the results attributed to twinning can be definitively associated with specific actions or interventions because the process is non-linear. Some results, both positive and negative, are unanticipated outcomes of twinning. For example, one twin pair in Sierra Leone was responsible for operationalising an ambulance service in a rural area. During the period of the project, the maternal mortality rate declined, but we must hasten to add that a variety of other conditions also changed in that period, including the building of a new road from the capital Freetown to the rural areas. This co-evolution made transport to hospitals easier for pregnant women, clearly another factor in the declining mortality.

Participating twins are all adults and leaders in their own right who will not always conform to hierarchical structures or agreements of the group at large. Self-organisation, feedback loops, non-linearity and unpredictability appear and re-appear continuously in twinning, and recognising these four elements of complexity gives insights into the dynamics at play (Table 4). 
Table 4. Four unexpected elements: from a taxonomy for complexity theory in healthcare (adapted from ${ }^{(5)}$.

$\checkmark \quad$ Self-organisation: Agents can adapt spontaneously to different challenges without hierarchical regulation. Feedback loops: Interacting agents cause both positive and negative effects on each other and the contex which affects their further behaviour.

Non-linearity: Small changes can have large, non-proportional effects that are directly evident. The effect can cause a 'tipping point' changing the present situation.

$\checkmark$ Unpredictability: Random, unplanned occurrences appear and have an unpredictable effect. Accurate, detailed planning is impossible.

Together with our concept analysis and our identification of the critical success factors, the twins who participated in the collaborations studied here confirm the centrality of reciprocity as a core value of twinning. Reciprocity is, by its very nature, non-linear. It can be seen from the focus groups and in-depth interview data that twins continuously struggled with the tri-partite process of giving, receiving, and reciprocating. Having something to offer gives power over the receiver, who must reciprocate or barter, either consciously or unconsciously, to balance the power between them ${ }^{[17]}$. Feedback loops, where twins adapt to each other during bartering, is integral to this process. This applies to both group and individual relationships. The struggle with this process is both a strength and a potential weakness of twinning.

As an example, twin pairs in the Dutch-Morocco collaboration happened to develop a higher number of small midwifery projects for the Moroccan context. Even though the Dutch group was part of this process, they felt increasingly disadvantaged by this imbalance and justified their more frequent - self-funded - travel to Morocco as a way to redress the balance. These trips were all self-organised, the project management team was rarely consulted, and it gave an extra dimension to the collaboration with many unintended results, such as the organisation of workshops in Morocco about vertical positions during birth. Predicting or trying to plan for these self-organised activities was impossible and even inappropriate as twins took the lead, which is a main goal of twinning. Twins also produced many unplanned products such as wrapping cloths printed with drawings about malaria prevention, badges to encourage blood donation, research published in international journals about diabetes in pregnancy, the introduction of birthing stools including evidence-based classes for midwives for their use, and stress management tools for student midwives, to mention but a few.

While our third (focus group) study shows that twinning results in the overall professional growth of twins, it is important to note that this was influenced by an intangible combination of personal values and cultural norms within a non-linear context that was constantly changing. The dynamics within the groups of twins from Morocco and the Netherlands show many feedback loops as twins, individually and in groups, discussed, reacted, and adapted to different actions and activities, resulting in unpredictable positive and negative responses. One telling example is of a photo of a not fully dressed woman in a hammam (public steam baths) that was visited by some twins, shared in the group WhatsApp. The visit was self-organised and the shared photo caused initial mayhem between and within the two groups. A public apology plus a discussion about privacy resulted in the positive development of clear social media rules as well as a negative development of lasting distrust between several twins.

Our study of the one-to-one twin relationships (Chapter 5) found that twin pairs navigated the complexity of twinning by building trusting relationships, allowing them to move forward $[18,19]$. Some pairs, however, were unable to build trusting relationships. It appears that self-organisation, where twins pro-actively adapt to the circumstances, plays an important role in this process, but here too, it is difficult to predict outcomes. We also found that a balance of power between twins influenced success and facilitated personal and professional flourishing. Yet failure lurked in the form of the temptation for both twins to regress into a classical aid mindset, characterised by an imbalance of power as a result of 'Northern' partner domination ${ }^{[20,21]}$. Twinning is not aid as classically conceived, because twinning invites participants to discover and equitably exchange their own resources ${ }^{[22]}$, strengthening both twins ${ }^{[23]}$. In this way, twinning is an innovative answer to the critique that international aid keeps the poor in the grip of the rich ${ }^{[20,24]}$. In the day-to-day dynamics of twinning, feedback loops influenced the continuous juggling of this power balance by twins. Their pro-active adaptation and self-organisation was crucial to success.

Using a complexity lens to understand twinning may make implementing this type of collaboration seem a daunting task, but it is reassuring to know that, like simple and complicated issues, twinning as a complex process can be approached with a degree of optimism ${ }^{[15]}$. After all, it can be complex to facilitate growth and agency in midwives, but we can look forward to working on these for the good of midwifery globally and be inspired while doing it.

\section{STRENGTHS AND LIMITATIONS}

Even though the positive potential of twinning is evident from our four studies, and the conclusions of many studies on twinning between midwife associations ${ }^{[1-4,11,18-24]}$, twinning is not a panacea for global human (health)inequities. 
This thesis constitutes four separate qualitative studies giving a wide as well as an in-depth approach to exploring twinning collaborations between associations of midwives. All authors have extensive backgrounds in qualitative research. The first author was deeply immersed in managing twinning collaborations, and all other authors have gained a substantial familiarity with the concept of twinning. Creating an open, respectful, and confidential atmosphere for participants, specifically for the studies presented in chapters 4 and 5 , was a high priority for the research team. However, the familiarity of the first author in particular, and to some extent, the second author, may have hindered some participants from expressing themselves freely. Added to this, the likely cultural bias due to the 'Western' perspective of the whole researcher team, may have influenced the research design, process and analysis. In terms of our analysis, the two experienced researchers on the team were not directly involved in the twinning collaborations under study, which allowed a more objective view of the data.

While many twinning collaborations exist globally, this is the first time the concept of twinning has been analysed and the first time an operational definition was developed. We analysed 19 publications on twinning in various sectors, making our definition broadly applicable. Twinning was the main search term we used, a possible limitation, as it may be that we excluded collaborations that worked according to twinning principles without using the name twinning.

In our Delphi study we used Rockart's four Critical Success Factors categories, originally developed for the business world. While this allowed us to illuminate aspects of twinning, it required a certain amount of interpretation to fit the factors identified by the 33 midwife twinning experts into these pre-existing categories. The final classifications were reached after rigorous discussion and agreement within our research group to minimize bias. Furthermore, four of the 12 non-respondents in our Delphi study were from low-income countries, diminishing the representativeness of our findings. We were aware that insufficient command of English, unreliability of internet access, and cultural challenges influenced both the response rate and the ability to be critical of the ranking process. All four countries of the UK were involved in the twinning collaborations used in our Delphi study, creating the potential for bias ${ }^{[25]}$. However, we think the potential for bias is limited because of the density of the information collected, the focused aim of our study, the inclusion of experts from diverse backgrounds, and the quality and detail of the comments provided [26]. We were unable to validate our initial questionnaire, but the validity and reliability of the overall study was strengthened by the use of the independent and broad responses of the experts to the open questions in the first round, which then became the basis for the statements in the second and third rounds [27]
Our two qualitative studies (Chapters 4 and 5) include rich and extensive data from participants in low, middle, and high-income countries from Europe, Northern Africa and Sub-Sahara Africa, adding to the global applicability of these studies. We were able to reaffirm the categories emerging from our data after saturation was reached midway through the analysis of both studies. The longitudinal aspect of the study using in-depth interviews (Chapter 5) strengthens the reliability and applicability of these data. Despite purposeful sampling, a bias was likely, as twins in the in-depth interviews mainly shared their successes even though they had been explicitly invited to share both positive and negative experiences and had also been guaranteed their confidentiality. This positive framing applied equally to the Dutch, Moroccan, and Sierra Leone midwife participants indicating that culture may not be the main cause. In the focus groups, cultural group norms did appear to influence the degree of positive and negative feedback, with the Moroccan focus groups being overwhelmingly positive and the Dutch focus groups slanting towards the negative. The familiarity of the first author as project manager of these twinning collaborations likely had an influence on the in-depth interviews and, to a lesser extent, on the focus groups. On the other hand this is also a strength of qualitative research, allowing deep familiarity with the context ${ }^{22,29]}$. Not unimportantly, language barriers meant that a professional interpreter was necessary for some of the in-depth interviews and focus groups. Some nuance may have been lost during translation.

Finally, although we studied several dimensions of twinning, the generalisability of the outcomes of this thesis for other (healthcare) professionals and the influence of other factors, like gender, remain unexplored.

\section{RECOMMENDATIONS FOR RESEARCH}

Our thesis has given a broad view of how twinning works including the factors that facilitate and hinder success, yet much is left unanswered. In reflecting on our research we developed four research recommendations.

1) Our study of twinning was limited to collaborations between midwives, all of whom were women. There is a need to investigate how twinning works for other healthcare professionals, including professions with gender diversity.

2) Our research looked at twinning between midwives from countries with substantial cultural, geographic, and income gaps. Additional research is needed to examine twinning between those from like-situated contexts.

3) Given our positive findings, a business case for twinning, with specific reference to its sustainability, including the possibility of micro financing, would be valuable. This study 
needs to take into account the broad range of benefits and costs of twinning, including professional and personal growth, the cost and benefit of volunteerism, the exchange of 'in kind' commodities, and other non-tangible attributes.

4) To gain more insight into the contextual and generalisable aspects of twinning we recommend a study of a project that uses the critical success factors as well as a complexity lens at the outset - including implementation, monitoring, adaptation, and evaluation.

\section{RECOMMENDATIONS FOR PRACTICE}

In light of the findings of, and reflections on, our four studies, we have developed six recommendations to facilitate the agency of midwives through twinning.

1) Implement twinning between associations of midwives globally to enhance the agency of midwives to play a key role in sexual reproductive healthcare.

2) Adhere to the new operational definition of twinning and pick the critical success factors that work for a specific context, as this will support and uphold the shared values of reciprocity and equity and keep your unified focus on a joint goal.

3) Be aware of and take into account the powerful influence of contextual differences between twin partners such as differences in culture, income category, geographical region, and language.

4) Incorporate one-to one twin pairs in the twinning collaboration as they can pave the way for success by moving beyond culture to the personal level, protecting the group from the damaging effects of stereotyping.

5) Take into account that twinning is a complex dynamic process during implementation, monitoring, adaptation, and evaluation processes by asking yourself non-linear, process questions and allowing space for a change of direction when needed. This gives unplanned additional developments the opportunity to emerge as part of the outcomes and enhances the overall chances of success.

6) Last but not least, celebrate successes consciously and visibly and remain optimistic during challenges remembering the ultimate goal. Twinning can promote the agency of midwives to do what they are best at - upholding and promoting sexual and reproductive human rights of women and giving quality and respectful midwifery care globally.

\section{CONCLUSION}

This thesis provides broad evidence for twinning and explores the complex process of twinning, offering a realistic and effective way of strengthening the agency of midwives globally. Twin pairs can give twinning an extra beneficial dimension by paving the way to success and overcoming problems of human prejudice and intolerance. Twinning is not predictable, or generalisable. This means that a 'one size fits all' strategy will not work. Instead, by allowing twinning to grow as a complex, dynamic process, while at the same time focusing - but not rigidly hanging on to - shared values and joint, inspiring goals, twinning can bring success and may even result in unintended positive outcomes. Setting up twinning collaborations between associations of midwives globally is a promising and dynamic process for enhancing the agency of midwives. 


\section{REFERENCES}

1. International Confederation of Midwives (ICM). (2014). Twinning manual. Editor Moyo N. T. the Hague.

2. Anderson, R. A., Crabtree, B. F., Steele, D. J., \& McDaniel Jr, R. R. (2005). Case study research: The view from complexity science. Qualitative health research, 15(5), 669-685. https://doi.org/10.1177/1049732305275208.

3. Capra, F. (2005). Complexity and life. Theory, Culture \& Society, 22(5), 33-44. https://doi. org/10.1177/0263276405057046

4. Cilliers, P. (2002). Complexity and postmodernism: Understanding complex systems. Routledge. https://doi. org/10.4324/9780203012253.

5. Nieuwenhuijze, M., Downe, S., Gottfreðsdóttir, H., Rijnders, M., du Preez, A., \& Rebelo, P. V. (2015). Taxonomy for complexity theory in the context of maternity care. Midwifery, 31(9), 834-843. https://doi.org/10.1016/j. midw.2015.05.009.

6. Plsek, P. E., \& Wilson, T. (2001). Complexity, leadership, and management in healthcare organisations. Bmj, 323(7315), 746-749. doi: https://doi.org/10.1136/bmi.323.7315.746.

7. Ramaswamy, R., Reed, J., Livesley, N., Boguslavsky, V., Garcia-Elorrio, E., Sax, S.,... \& Parry, G. (2018). Unpacking the black box of improvement. International Journal for Quality in Health Care, 30(suppl_1), 15-19. https://doi.org/10.1093/intahc/mzy009.

8. Bucknall, T., \& Hitch, D. (2018). Connections, Communication and Collaboration in Healthcare's Complex Adaptive Systems: Comment on" Using Complexity and Network Concepts to Inform Healthcare Knowledge Translation". International journal of health policy and management, 7(6). 556. doi: 10.15171/iihpm.2017.138.

9. World Health Organization (WHO), International Confederation of Midwives (ICM), White Ribbon Alliance (WRA). (2016). Midwives voices, midwives realities. Findings from a global consultation on providing quality midwifery care. ISBN 9789241516112

10. United Nations Population Fund (UNFPA). (2014) The State of the World's Midwifery, a universal pathway. A woman's right to health. Editor Bernis L.D. UNFPA, ICM, WHO: New York. ISBN: 978-0-89714-026-3 Available from: http://www.unfpa.org/sowmy

11. Walsh, D. M. Christianson, and Stewart M. (2015). Why midwives should be feminists. MIDIRS Midwifery Digest, 2015. 25(2): p. 154-160. MIDIRS Midwifery Digest. 25(2):154-160.

12. Coe, I. (2019). Feminism is for everybody. The Lancet, 393(10171), 493. https://doi.org/10.1016/501406736(19)30239-9.

13. International Confederation of Midwives (ICM). (2017). Definitions. [cited 2020]. Available from: https://www. internationalmidwives.org/our-work/policy-and-practice/icm-definitions.html.

14. Cadée, F., Nieuwenhuijze, M. J., Lagro-Janssen, A. L. M., \& De Vries, R. (2016). The state of the art of twinning, a concept analysis of twinning in healthcare. Globalization and health, 12(1), 66. doi: 10.1186/s12992-016$0205-5$.

15. Glouberman, S., \& Zimmerman, B. (2002). Complicated and complex systems: what would successful reform of Medicare look like?. Romanow Papers, 2, 21-53. Toronto Canada. ISBN 0-8020-8616-7.

16. Cadée, F., Nieuwenhuijze, M. J., Lagro-Janssen, A. L., \& de Vries, R. (2018). From equity to power: Critical
Success Factors for Twinning between midwives, a Delphi study. Journal of advanced nursing, 74(7), 15731582. https://doi.org/10.1111/jan.13560

17. Kowalski. R. (2011). The Gift-Marcel Mauss and international aid. Journal of Comparative Social Welfare, 27(3) 189-205. https://doi.org/10.1080/17486831.2011.595069.

18. Möllering, G. (2001). The nature of trust: From Georg Simmel to a theory of expectation, interpretation and suspension. Sociology, 35(2), 403-420. https://doi.org/10.1017/S0038038501000190.

19. Vangen, S., \& Huxham, C. (2003). Nurturing collaborative relations: Building trust in interorganizational collaboration. The Journal of Applied Behavioral Science, 39(1), 5-31. https://doi.org/10.1177/0021886303 039001001.

20. Moyo, D. (2009). Dead aid: Why aid is not working and how there is a better way for Africa. Macmillan. ISBN 978-0-141-03118-7.

21. Anderson, F., Donkor, P., de Vries, R., Appiah-Denkyira, E., Dakpallah, G. F., Rominski, S.... \& Rana, G. K. (2014). Creating a charter of collaboration for international university partnerships: The Elmina Declaration for Human Resources for Health. Academic Medicine, 89(8), 1125-1132. doi: 10.1097/ACM.0000000000000384.

22. Stirrat, R. L., \& Henkel, H. (1997). The development gift: The problem of reciprocity in the NGO world. The Annals of the American Academy of Political and Social Science, 554(1), 66-80. https://doi.org/10.1177/0002 716297554001005.

23. Cadée, F., Perdok, H., Sam, B., de Geus, M., \& Kweekel, L. (2013). 'Twin2twin'an innovative method of empowering midwives to strengthen their professional midwifery organisations. Midwifery, 29(10), 1145-1150. htps://doi.org/10.1016/.midw.2013.07.002

24. Chang, H. J. (2010). Bad Samaritans: The myth of free trade and the secret history of capitalism. Bloomsbury Publishing USA. ISBN10 1596915986

25. Royal College of Midwives (RCM). (2015). Global midwifery twinning project. [cited 2020] Available from: https:// www.rcm.org.uk/promoting/global/projects/global-midwifery-twinning-project/.

26. Malterud, K., Siersma, V. D., \& Guassora, A. D. (2016). Sample size in qualitative interview studies: guided by information power. Qualitative health research, 26(13), 1753-1760. https://doi.org/10.1177/1049732315617444.

27. Keeney, S., Hasson, F., \& McKenna, H. (2006). Consulting the oracle: ten lessons from using the Delphi technique in nursing research. Journal of advanced nursing, 53(2), 205-212. https://doi.org/10.1111/j.13652648.2006.03716.x.

28. Pawluch, D., \& Neiterman, E. (2010). What is grounded theory and where does it come from. The SAGE handbook of qualitative methods in health research, 174-192. ISBN 978-1-84787-292-0.

29. Levy, V. (1999). Protective steering: a grounded theory study of the processes by which midwives facilitate informed choices during pregnancy. Journal of advanced nursing, 29(1), 104-112. doi:10.1046/j.13652648.1999.00867.x. 


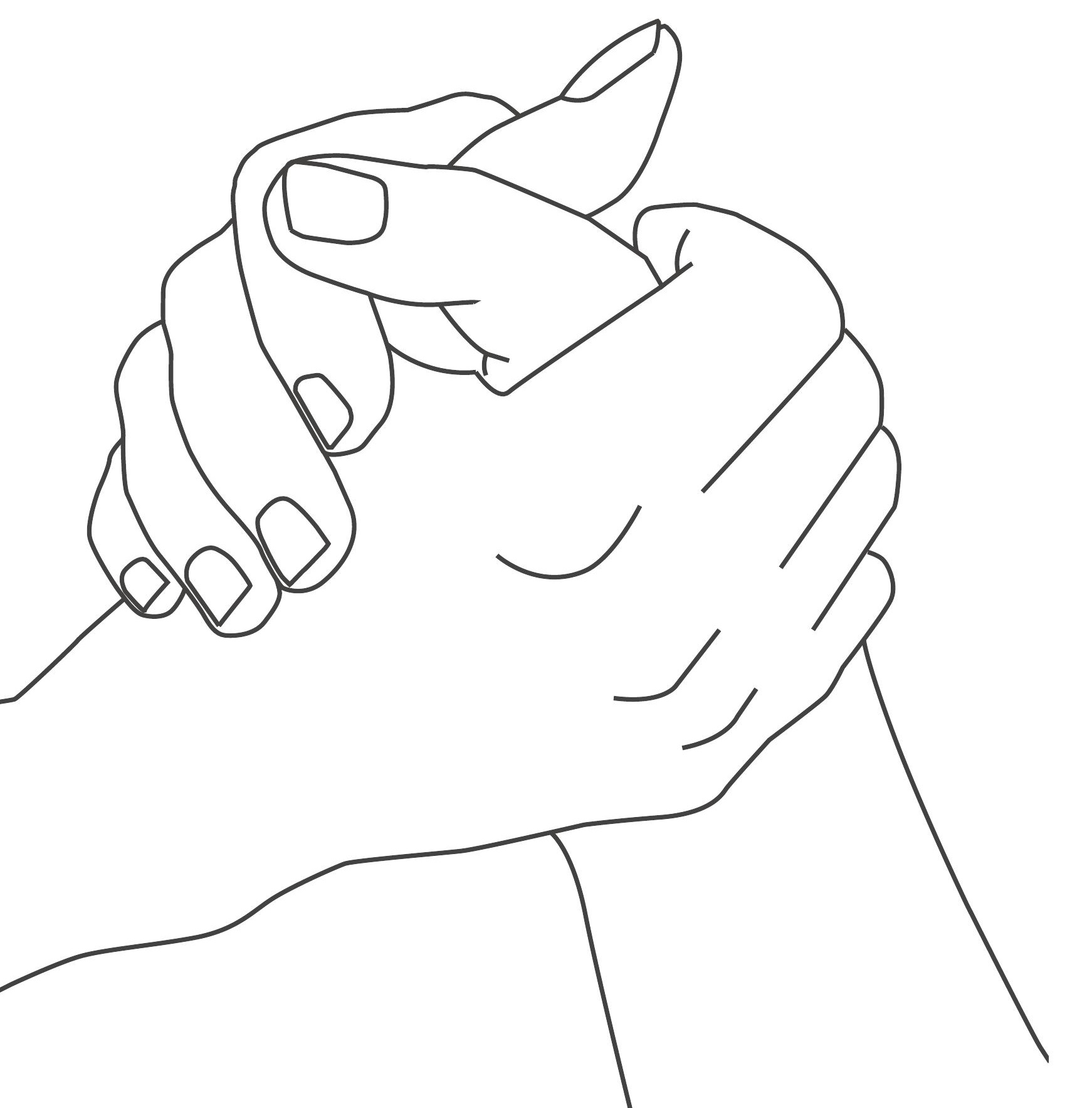

\section{Summary in English}

Twinning, a promising dynamic process to strengthen the agency of midwives

\section{Franka Cadée}

Marianne J. Nieuwenhuijze

Antoine L.M. Lagro-Janssen

Raymond De Vries 


\section{SUMMARY}

\section{CHAPTER 1}

This introductory chapter sets the scene for further exploration of twinning in healthcare with specific reference to twinning between associations of midwives.

Global inequities in health have garnered international attention and are now addressed in Sustainable Development Goals 3 and 5, which seek to 'promote well-being for all' and 'achieve gender equality and to empower all women and girls' by 2030. With just one decade left to attain these goals, a certain urgency calls for innovative and evidence based approaches.

International publications identify midwives as key for improving maternity care worldwide. Yet patriarchal models have resulted in the lack agency of midwives to take on this role. Twinning, where two groups - from educational institutions, hospitals or towns - work together cross-culturally on joint goals, have garnered potential to develop agency in professionals, but as yet, there is no empirical evidence or theory that offers insight into their value. Even though twinning collaborations, are increasingly common worldwide, a stronger evidence base is needed in order to understand whether twinning has its promised potential, and, if so, to develop a clearer understanding of what twinning is, how it works, and how to optimise its potential. The four studies plus the general discussion in this thesis address this knowledge gap.

\section{CHAPTER 2}

Because twinning in healthcare is relatively new, the definition and understanding of twinning lacks clarity and rigorous monitoring and evaluation are rare, we conducted a concept analysis (CA) of twinning in healthcare using Morse's method. For this a qualitative study of the broad literature was performed, including scientific papers, manuals, project reports, and websites. We identified relevant papers through a systematic search using scientific databases, backtracking of references, and experts in the field.

We found nineteen papers on twinning in healthcare. This included twelve peer reviewed research papers, four manuals on twinning, two project reports, and one website. Seven of these papers offered no definition of twinning. In the other twelve papers definitions varied. Our CA of the literature resulted in four main attributes of twinning in healthcare. First, and most frequently mentioned, was reciprocity. The other three attributes were that twinning: 2) entails the building of personal relationships, 3) is a dynamic process, 4) is between two named organisations across different cultures. The literature also indicated that these four attributes, and especially reciprocity, can have an empowering effect on healthcare professionals.

Based on these four attributes we developed the following operational definition: 'Twinning is a cross-cultural, reciprocal process where two groups of people work together to achieve joint goals'. A greater understanding and a mature definition of twinning results in clear expectations for participants and thus more effective twinning. This can be the starting point for new collaborations and for further international studies on the effect of twinning in healthcare.

\section{CHAPTER 3}

In this study we aimed to gain consensus for Critical Success Factors associated with twinning in midwifery. Between 2016-2017, we approached 56 midwife twinning experts from 19 countries to participate in a Delphi study consisting of three rounds.

In round one, experts gave input through an open ended questionnaire and this was analysed to formulate Critical Success Factors statements that were scored on a 1-7 Likert scale aiming to gain consensus in round 2 and 3 . These statements were operationalised for practical use such as a check list in planning, monitoring and evaluation in the field.

Thirty-three experts from 14 countries took part in all three Delphi rounds, producing 58 initial statements. This resulted in 25 Critical Success Factors (CSF) covering issues of management, communication, commitment and values. Most of these CSF's focus on equity.

From this we concluded that the Critical Success Factors formulated in this study, represent the necessary ingredients for successful twinning by providing a practical implementation framework, and promote further research into the effect of Twinning. The findings show that making equity explicit in twinning may contribute towards the agency of midwives to take on their identified key role in sexual and reproductive healthcare.

\section{CHAPTER 4}

In this chapter we explored how the professional growth of midwives, essential for optimising midwifery agency globally, can be enhanced through twinning collaborations. We payed specific attention to how professional growth is affected by cultural differences between twins. This study was performed by means of a longitudinal qualitative design including data from open-ended questionnaires and focus groups. These data were analysed using content analysis. 
Our findings show that cultural differences were capable of both hindering and facilitating professional growth. Within the complex dynamics of twinning, professional growth was facilitated by twins' preparedness to bridge cultural differences. Common goals positively influenced this process. Friction was more likely, and professional growth was hindered, when midwives were unprepared to bridge cultural differences. To conclude, we recommend a clear focus on common goals and consideration of the interaction between the length of a project and the extent of the cultural differences between twins, to optimise professional growth through twinning.

\section{CHAPTER 5}

The aim of this fourth study was to explore the contribution of one-to-one relationships between twins to twinning projects, as exemplified in projects between Dutch and Moroccan, and Dutch and Sierra Leone midwives. For this we conducted thirteen in-depth interviews with midwives from two twinning collaborations. Interviews were transcribed and analysed using an iterative, grounded theory process, yielding a theoretical understanding of one-toone twinning relationships for twinning collaborations.

We found that participant comments fell into four substantive categories: 1) Being named a twin, 2) moving beyond culture to the personal level, 3) searching for common ground to engage, 4) going above and beyond the twinning collaboration. Their interplay demonstrates the value of twin pairs in paving the way for successful twinning.

A complex combination of contextual inequities, personality, and cultural differences affect the twin relationship. Trusting relationships promote effective collaboration; however, as 'trust' cannot be mandated, it must be built by coaching twins in personal flexibility and (cultural) communication. By offering original insights into the ways twinning relationships are built, our research explores how twin pairs can enhance the success of twinning projects.

\section{CHAPTER 6}

In the final general discussion of our four studies we reflect on the main findings using a complexity theory lens, a useful tool for examining the reality of twinning in the field - that is, how twinning actually works. We also consider the limitations and strengths of the thesis and provide recommendations for future research and for practice.

During the five years of our research, it became clear that successful twinning is not a linear process. Twinning is not predictable, or generalisable. This means that a 'one size fits all' strategy will not work. Instead, by allowing twinning to grow as a complex, dynamic process, while at the same time focusing - but not rigidly hanging on to - shared values and joint, inspiring goals, twinning can bring success and may even result in unintended positive outcomes. We concluded that an understanding of the complexity of the twinning process, supports successful twinning collaborations between associations of midwives globally as a promising and dynamic process for enhancing the agency of midwives. 


\section{SAMENVATTING}

\section{HOOFDSTUK 1}

Dit inleidende hoofdstuk schetst de achtergrond van 'twinning' in de gezondheidszorg met een specifieke verwijzing naar twinning tussen verenigingen van verloskundigen.

Wereldwijde ongelijkheden op het gebied van gezondheid krijgen internationale aandacht en worden nu behandeld in de duurzame ontwikkelingsdoelstellingen (SDG) 3 en 5, die vóór 2030 'welzijn voor iedereen' willen bevorderen en 'gendergelijkheid en empowerment willen bereiken voor alle vrouwen en meisjes'. We hebben nog maar tien jaar om deze doelen te bereiken. Daarom is er dringend behoefte aan innovatieve en bewezen effectieve benaderingswijzen

Internationale publicaties wijzen een sleutelrol toe aan verloskundigen om moeder- en kindzorg wereldwijd te verbeteren. Patriarchale systemen hebben er echter toe geleid dat verloskundigen onvoldoende zelfbeschikking hebben om deze rol optimaal te kunnen vervullen. Twinning, waarbij twee groepen - van onderwijsinstellingen, zorginstellingen of steden - transcultureel samenwerken aan gezamenlijke doelen, heeft aangetoond de potentie te hebben om deze zelfbeschikking bij professionals verder te ontwikkelen. Vooralsnog is er echter geen empirisch bewijs of theorie om aan te tonen hoe waardevol twinning nu werkelijk is. Hoewel twinningsamenwerkingsverbanden wereldwijd steeds gebruikelijker zijn, is er een sterkere wetenschappelijke basis nodig om te begrijpen of twinning het beloofde potentieel kan realiseren. Verder is er behoefte aan een beter begrip van wat twinning is, hoe het werkt en hoe we het optimaal kunnen toepassen.. De vier studies in deze thesis, samen met de algemene bespreking, behandelen deze kenniskloof.

\section{HOOFDSTUK 2}

Twinning in de gezondheidszorg is relatief nieuw. Daarom is het geen verrassing dat de definitie en het begrip van twinning onduidelijk zijn en dat twinningsamenwerkingsverbanden in het veld zelden grondig worden gepland, gemonitord en geëvalueerd. Als reactie op deze lacune hebben we een conceptanalyse (CA) van twinning in de gezondheidszorg uitgevoerd. Volgens de methode van Morse hebben we de literatuur, waaronder wetenschappelijke artikelen, handleidingen, projectrapporten en websites, kwalitatief geanalyseerd. We hebben relevante artikelen geïdentificeerd door middel van systematisch onderzoek met behulp van wetenschappelijke databases, back-tracking van referenties en overleg met experts in het veld.

We hebben negentien publicaties over twinning in de gezondheidszorg gevonden. Deze bestonden uit twaalf wetenschappelijke artikelen, vier handleidingen over twinning, twee projectrapporten en één website. Zeven van deze publicaties boden geen definitie van twinning. In de andere twaalf publicaties liepen de definities uiteen. Onze CA van de literatuur resulteerde in vier hoofdkenmerken van twinning in de gezondheidszorg. Het eerste en meest genoemde kenmerk was wederkerigheid. De andere drie kenmerken waren: 2) twinning omvat het opbouwen van persoonlijke relaties, 3) twinning is een dynamisch proces, 4) twinning vindt plaats tussen twee genoemde organisaties van verschillende culturen. De literatuur gaf ook aan dat deze vier kenmerken, en met name wederkerigheid, een versterkend effect kunnen hebben op het vermogen tot zelfbeschikking van zorgverleners. Op basis van deze vier kenmerken hebben we de volgende werkdefinitie van twinning ontwikkeld: 'Twinning is een intercultureel, wederkerig proces waarbij twee groepen mensen samenwerken om gezamenlijke doelen te bereiken'. Een beter begrip en een verder ontwikkelde definitie van twinning leiden tot duidelijke verwachtingen bij deelnemers en dus tot een effectievere twinning. Ons werk biedt een startpunt voor nieuwe samenwerkingen en voor verder internationaal onderzoek naar het effect van twinning in de gezondheidszorg.

\section{HOOFDSTUK 3}

In deze studie wilden we consensus bereiken over de kritische succesfactoren voor twinning in de verloskunde. Tussen 2016-2017 hebben we 56 verloskundigen die twinningexperts waren uit 19 landen uitgenodigd om deel te nemen aan een Delphi-onderzoek bestaande uit drie rondes.

In de eerste ronde leverden experts input door middel van een open vragenlijst, die vervolgens werd geanalyseerd om stellingen over kritische succesfactoren te formuleren. In de tweede en derde ronde werden deze stellingen beoordeeld met behulp van een Likertschaal om de mate van consensus onder de experts te meten. Tenslotte werden deze stellingen geoperationaliseerd voor praktisch gebruik, waaronder de ontwikkeling van een checklist voor planning, monitoring en evaluatie in het veld.

Drieëndertig experts uit 14 landen namen deel aan alle drie de Delphi-rondes en produceerden 58 eerste stellingen. Na de tweede en derde ronde werd de lijst teruggebracht tot 25 kritieke succesfactoren die betrekking hebben op management, communicatie, betrokkenheid en waarden. De meeste factoren zijn gericht op gelijkwaardigheid. We concludeerden dat de kritieke succesfactoren die in deze studie zijn geformuleerd de noodzakelijke ingrediënten zijn voor succesvolle twinning omdat ze een praktische handleiding vormen om de implementatie en verder onderzoek naar het effect van twinning te bevorderen. De bevindingen tonen aan dat het expliciet benoemen van gelijkwaardigheid in twinning verloskundigen kan versterken, waardoor zij hun erkende en belangrijke rol in de seksuele 


\section{HOOFDSTUK 4}

In dit hoofdstuk hebben we onderzocht hoe twinning eraan kan bijdragen dat verloskundigen steeds professioneler worden, iets wat essentieel is voor het wereldwijd optimaliseren van verloskundigenzorg. We hebben speciale aandacht besteed aan hoe professionele groei wordt beïnvloed door culturele verschillen tussen twins. Deze studie werd uitgevoerd door middel van een longitudinale, kwalitatieve onderzoeksopzet op basis van gegevens uit open vragenlijsten en focusgroepen. Deze gegevens werden geanalyseerd met behulp van inhoudsanalyse.

Onze bevindingen tonen aan dat culturele verschillen professionele groei zowel konden belemmeren als faciliteren. Binnen de complexe dynamiek van twinning werd professionele groei mogelijk gemaakt doordat de twins bereid waren om culturele verschillen te overbruggen. Gemeenschappelijke doelen hadden een positieve invloed op dit proces. Als verloskundigen niet bereid waren om culturele verschillen te overbruggen trad vaker wrijving op en werd professionele groei belemmerd. We bevelen tenslotte aan dat er voor het optimaliseren van professionele groei door middel van twinning een duidelijke focus moet zijn op gemeenschappelijke doelen, en dat er een connectie gemaakt moet worden tussen de lengte van een project en de omvang van de culturele verschillen tussen twins.

\section{HOOFDSTUK 5}

In onze vierde studie onderzoeken we de bijdrage van één-op-één relaties tussen twinparen aan twinningsamenwerkingsverbanden, zoals geillustreerd in samenwerkingsverbanden tussen Nederlandse en Marokkaanse en Nederlandse en Sierra Leonese verloskundigen. Hiervoor hebben we dertien diepte-interviews gehouden met verloskundigen uit deze twee twinning samenwerkingsverbanden. De interviews werden getranscribeerd en geanalyseerd door middel van een iteratief, gefundeerd theorieproces, dat theoretisch inzicht opleverde in de waarde van één-op-één twinningrelaties voor twinningsamenwerkingsverbanden.

We ontdekten dat de opmerkingen van de deelnemers in vier inhoudelijke categorieën vielen: 1) erkend worden in hun rol als twin, 2) naar het persoonlijke vlak kunnen gaan, cultuur overstijgend, 3) zoeken naar een gemeenschappelijke basis om deel te nemen, 4) verder willen gaan dan de kaders van het twinning-samenwerkingsverband. Het samenspel van deze vier elementen van twinning toont aan hoe waardevol het is dat twinparen de weg banen naar succesvolle twinning. Een complexe combinatie van contextuele ongelijkheden, persoonlijkheid en culturele verschillen beïnvloedt de twinrelatie. Vertrouwen bevordert effectieve samenwerking, maar omdat 'vertrouwen' niet kan worden afgedwongen moet het worden opgebouwd. Dit kan gebeuren door twins te coachen in persoonlijke flexibiliteit en (culturele) communicatie. Door creatieve inzichten te bieden in de manier waarop twinrelaties worden opgebouwd, toont ons onderzoek hoe twinparen het succes van twinningsamenwerkingsverbanden kunnen vergroten.

\section{HOOFDSTUK 6}

Het laatste hoofdstuk is een algemene bespreking van onze vier studies. In dit hoofdstuk reflecteren we op de belangrijkste bevindingen met behulp van een lens uit de complexiteitstheorie, een nuttig hulpmiddel om de realiteit van twinning in het veld te onderzoeken, oftewel te begrijpen hoe twinning nu eigenlijk werkt. We houden ook rekening met de beperkingen en sterke punten van het proefschrift en doen aanbevelingen voor toekomstig onderzoek en voor de praktijk.

Gedurende de vijf jaar van ons onderzoek werd duidelijk dat succesvolle twinning geen lineair proces is. Twinning is niet voorspelbaar en kan niet worden teruggebracht tot een reeks algemene stappen. Dit betekent dat een 'one size fits all' -strategie niet werkt. Door twinning te laten groeien als een complex, dynamisch proces, en tegelijkertijd te focussen op - maar niet strikt vast te houden aan - gedeelde waarden en gezamenlijke, inspirerende doelen, kan twinning succesvol zijn en zelfs leiden tot onbedoelde, positieve resultaten. We concluderen dat een goed begrip van de complexiteit van het twinningproces succesvolle twinningsamenwerkingsverbanden tussen verenigingen van verloskundigen wereldwijd ondersteunt en als zodanig een veelbelovende en dynamische strategie kan zijn voor het verbeteren van het zelfbeschikkingsrecht van verloskundigen. 


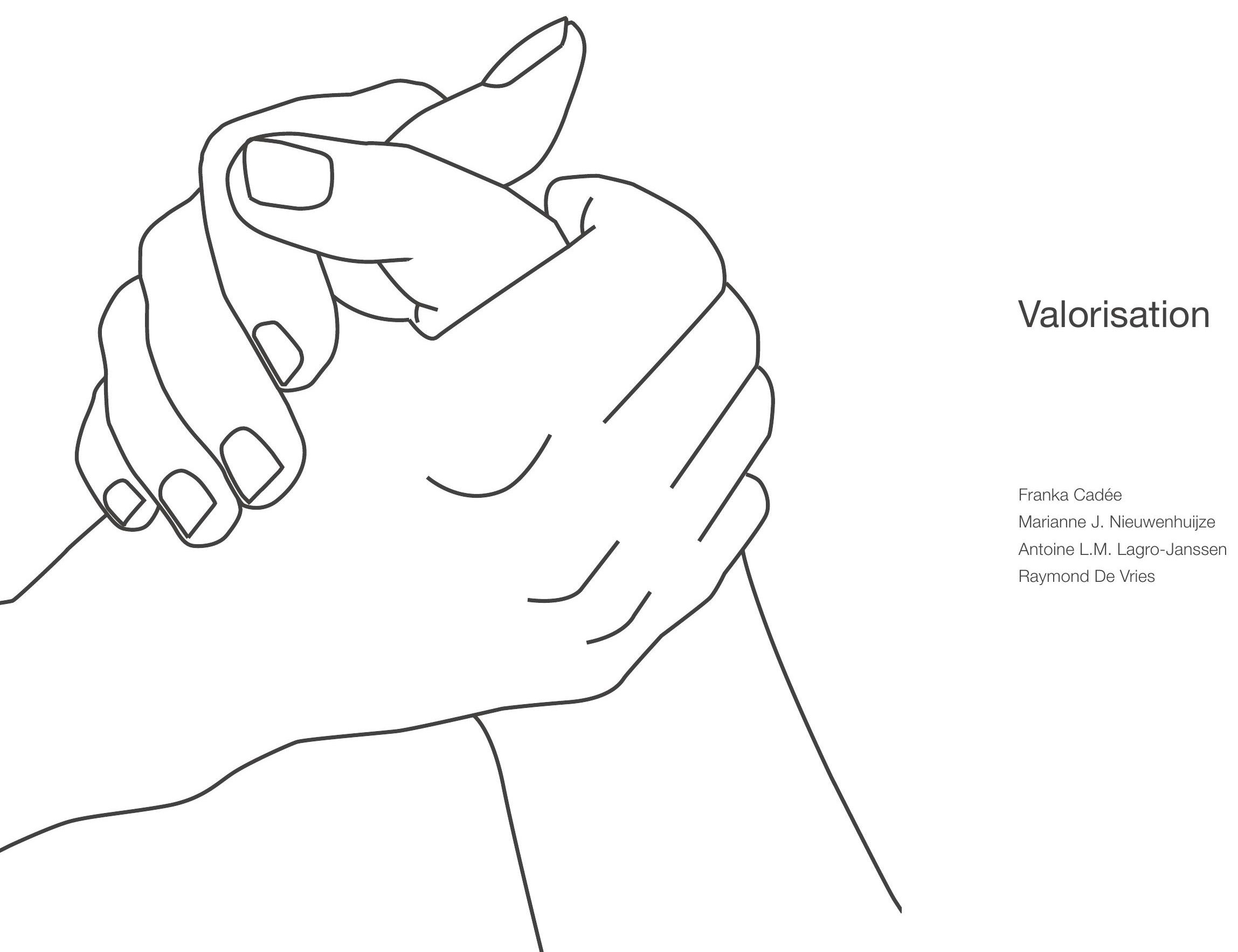


"Twinning has opened my eyes, I never knew that while we (midwives) live such different lives we face similar issues. It has given me the courage to speak up!' Twin, collaboration Sierra Leone \& the Netherlands.

\section{TIMELY RELEVANCE OF THIS THESIS}

It is easy to become complacent, thinking that great progress is being made in sexual and reproductive health and rights of women and girls globally. After all, grassroots and global campaigns are underway. The \#metoo movement is addressing issues of sexual harassment. The White Ribbon Alliance is looking @whatwomenwant via the largest ever open-ended survey of women's opinions about their needs for quality reproductive and maternal healthcare. The Women Deliver campaign, Deliver for Good, is encouraging investment in girls and women because it will 'power progress for all'. All these campaigns are urgently needed, but nevertheless, the fact is that at present, the provision of sexual and reproductive healthcare and the protection of the rights of girls and women are seriously threatened.

Most of the world's health workforce is female. The World Economic Forum's Global Gender Gap Report 2020 projects that, if current trends continue, the global gender gap will take 99.5 years to close. This has severe repercussions for the development and sustainability of the female health workforce. Just one of many gender aspects that undermines both midwives and nurses is the conflation of their professions. Whilst the work of a paediatrician is clearly delineated from the work of a cardiologist, as conflating these professions would endanger patients, women's work is just women's work. Midwifery and nursing are distinct professions but often, especially in low and middle income countries where staff shortages are common, midwives and nurses are expected to take over the tasks of the other. If we are to maintain a woman's right to receive skilled reproductive and sexual healthcare, it is important to maintain this distinction and to allow midwives to offer their expertise to women.

This year, 2020, has been proclaimed the year of the midwife and the nurse by the World Health Organization (WHO). The International Confederation of Midwives (ICM) is the united voice of midwives globally. The option of twinning, allowing midwives to support other midwives and to strengthen their agency to take the actions called for by women, comes at an opportune time. The focus of twinning on equity is in line with women's and midwives' call for equity. In the ICM member expectations and needs survey (2018), members explicitly asked for support to initiate twinning collaborations between midwife associations. There is a future for twinning. The four studies in this thesis offer the foundation for the successful realisation of this request by ICM midwife associations and beyond.

\section{RESEARCH HAND IN HAND WITH PRACTISE}

There has been a direct and constant connection between the four studies in this thesis and the running of the collaborations described here, as well as ongoing twinning projects. Outcomes from these four studies have been directly integrated into the approach of twinning collaborations as well as shared with others involved in twinning. In this way our research has contributed to new knowledge to strengthen midwives and improve international and intercultural collaboration. The research has also had implications for the choice of twinning partners as well as the way twinning collaborations have been planned and evaluated. Two examples are 1) the choice for twinning between Morocco and the Netherlands, where the shorter distance was considered to be an important factor, and 2) a new collaboration between the Netherlands and Iceland was chosen because of a smaller income gap. The latter twinning collaboration is still under study and the results will be published after completion of the project.

The potential of one-to-one twinning as described in chapter 5 has offered new insights to improve the positive impact of twinning. This innovation has challenged midwife associations globally involved in twinning to reconsider their own approach. The publication as well as presentation of our research has stimulated others involved in twinning to evaluate and publish their own insights and outcomes. This has resulted in a growing body of evidence and knowledge on twinning.

\section{WHO CAN BENEFIT FROM TWINNING?}

Twinning is not exclusive to midwives. Other professions, organisations, and groups can also benefit from creating twinning collaborations. Our new definition of twinning was formulated with the use of articles, papers, manuals and websites related to twinning between different healthcare professionals, allowing it to be applied to twinning between healthcare professionals in general. But our research does not preclude twinning between professionals outside the health sector, or even between different professionals. There is potential for twinning between teachers and nurses, midwives and lawyers, obstetricians and plumbers, academics and farmers to give but a few (wild) suggestions.

\section{ACTIVITIES}

Our four studies and past and ongoing twinning collaborations with Sierra Leone, Ghana, the Netherlands, Iceland and Morocco have brought us in contact with many specialists and organisations who are actively working in the field of strengthening healthcare workers, and specifically midwives. These include midwife associations from the UK, Sweden, Japan, Canada and Germany. These contacts have led to many spontaneous conversations and discussions about the do's and don'ts of twinning. Through the years our research team 
has given regular organisational support for the improvement of twinning collaborations globally. As a direct consequence of these conversations, there will be an inaugural meeting of midwife associations involved in international work and our four studies will be presented during a symposium at the International Confederation of Midwives (ICM) Triennial Congress in Bali, 2020. In addition, different practical aspects of setting up twinning collaborations as well as the results from our four studies have been presented at the following international congresses:

\section{ORAL PRESENTATIONS}

2019 Twinning in practise. Congress of the Nordic Federation of Midwives: Midwifery across borders. Reykjavik, Iceland.

2017 So we are Twinning, and then? International Confederation of Midwives (ICM) Triennial Congress, Toronto, Canada.

2016 What is twinning \& why should we care? Radboud Medical Centre, Nijmegen, the Netherlands.

2016 A Concept Analysis of twinning in healthcare. European Midwives Association (EMA) Education Conference, London, UK.

2015 Outcomes of twinning between Dutch \& Moroccan midwives. Colloquium for maternal \& neonatal health, Rabat, Morocco.

2014 Twinning an innovative method of co-development. International Confederation of Midwives (ICM) Triennial Congress, Prague, Czech Republic.

2012 Twinning in practice. National Conference to celebrate the day of the Midwife, Royal College of Midwives, London, UK.

2011 Twinning Sierra Leone \& the Netherlands. International Confederation of Midwives (ICM) Triennial Congress, Durban, South Africa. 


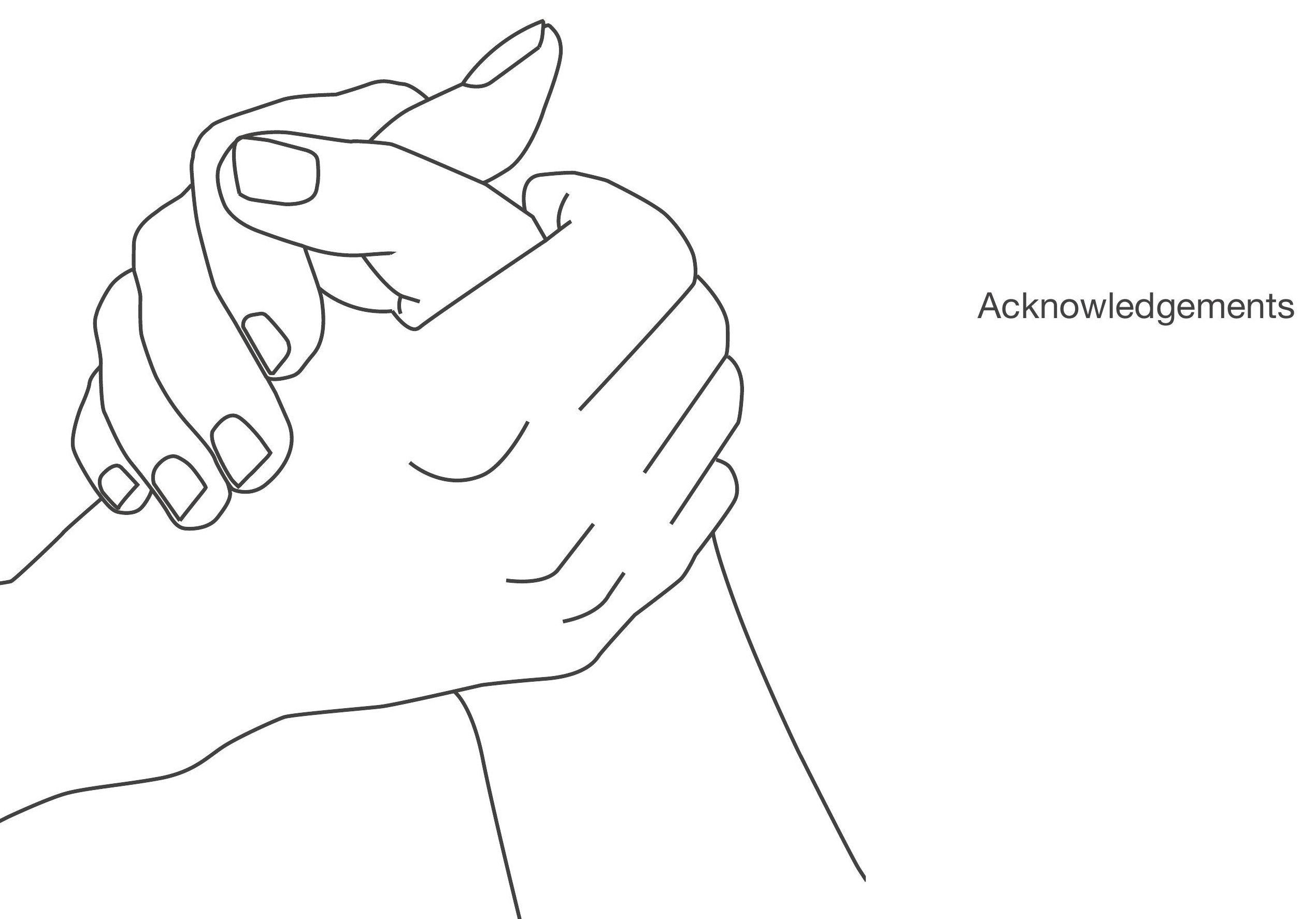




\section{THANK YOU!}

As a midwife, the 1st of May, labour day, seemed ideal for my PhD defence, however life is not predictable and the Covid-19 Pandemic together with my wish for a 'live' public defence followed by a party meant that the date was delayed by 5 months. Time has flown, and what started off as curiosity and passion, got out of hand and turned into a five year $\mathrm{PhD}$ period that has felt like a privilege and great learning experience for most of the time. I am deeply aware that it would not have been possible without the support of many very special people.

\section{OWE MY CREDIBILITY TO MY SUPERVISORS}

I remember the first discussion I had with my three supervisors, it was about having to 'kill my darlings', which I tried with limited success. At the same time I gathered many new darlings, my three supervisors are amongst them. We are a great team!

Thank you Marianne for you optimism, helping me distinguish twinning project issues from research issues, introducing me to your broad academic knowledge, being a great travel companion, supporting me with my interview skills, and your never ending patience.

Dear Toine, thank you for your gentle sharpness, for being a role model to me, pointing out my gender normative behaviour, your encouraging cards, helping me to see when things are, or aren't good enough, and your talent for catchy titles.

Raymond, your deep understanding of the significance of midwives for society has been inspirational, your abstract and clear thinking has keep me on my toes, I will always be envious of your writing skills, and never appearing to be irritated or stressed.

A special thanks to the Assessment and Defence Committee for giving your dedicated time and attention to my twinning research work. I do worry that you may realise that I am an imposter.

\section{OWE FEELING ACCEPTED AND SECURE TO MY FAMILY}

My gorgeous man and soulmate Jos has stood by my side with pride while my attention was often elsewhere, especially in the last year where the combination of my current job with my PhD writing, gave little room for anything else. Thank you for cooking all those meals and celebrating small victories together with beer by the fire. Thank you for waving me goodbye so often and then welcoming me back home again with open arms. Thank you Ella for the creative design of the cover of this thesis that would never have been pink without you. To Ella, Tobias and Benjamin, thank you for accepting and loving me for the mother and person I am.
Thank you Tessa, Mark, Jeroen and Iris for letting me to love my grandchildren Kate, Julia, Mats, $\mathrm{Mia}^{\dagger}$ and Sophie while I am so often not around.

Thank you to my parents and sister who explored the world with me when I was still very young which garnered in me an interest in life's colourful diversity and the value of equality.

\section{OWE MY SANITY TO MY FRIENDS}

A give a deep bow to the Sisters and my special friend, Mother Emiliani of the Monastere Orthodoxe Dame de Toute protection, for welcoming me every year, and to be a part of your community while doing my PhD writing. To Karin, Carin, Sjoukje and Saskia for listening to me, singing together and enjoying the much needed visits to the sauna. To Mary, for being my midwife not only at the birth of Ella, but also the birth of my PhD thesis by proofreading it so diligently. Thank you Nicola for teasing me and please, don't cheer on the Defence Committee like you do with football.

Thank you to Sjoukje and Liselotte for standing next to me during the PhD defence. I know you know I find it more impressive than I let on.

\section{OWE MY REASON FOR BEING TO MIDWIFERY}

Becoming a midwife was a life changing experience for me because it has given me a clear focus and the drive to bring to the world's and specifically women's attention, the value of midwifery for a healthy future. Through my twinning work and the studies in this PhD thesis, my anthropological background and midwifery came together.

I am very grateful to the $+\backslash-140$ midwives that took part in the different twinning projects, and who were willing to be part of a live experiment that was a great ride, but did not always succeed. Thank you for the inspirational team work and dedication of the twinning project teams from Sierra Leone: Betty \& Safiatu, Ghana: Gifty \& Netta, Morocco: Lislotte \& Fatima, the Netherlands/Iceland: Liselotte \& Edythe. Your personal dedication and support made this research possible.

Thank you to the midwives of my former midwife practise de Bakermat, for accommodating my whims, and selling close to 1000 baby hats to support the different twinning initiatives. Thank you for the inspiration I received from the many midwives I met during this journey and who all contribute to the wealth of knowledge, skills and the art of midwifery. I hope the International Confederation of Midwives can make use of this work to support midwives to be heard and listened to. 


\section{FRANKA CADÉE CURRICULUM VITAE}

1963

1969

1971

1974

1975

1975-2017

1979

1984

1988

1988

1988

1990

1990

1990

1992-2012

1996

2000

2002-2008

2004-2007

2005-2007

2007

2007-2010

Born at home, Voorburg, the Netherlands

Primary education, home schooling, Multan, Pakistan

Primary education, Roncalli School, Alphen a/d Rijn, the Netherlands

Secondary education, Unity High School, Khartoum, the Sudan

Secondary education (O levels), Downe House School, Cold Ash, the UK

Singer in various classical and pop choirs, the UK and the Netherlands

Secondary education (International Baccalaureate), St Claire's Hall,

Oxford, the Uk

BSc Cultural Anthropology, London School of Economics \& Politica

Science, London, the UK

State registered nurse, The Royal London Hospital Trust, London, the UK

Evening school, car mechanics, London, the UK

Birth of daughter Ella, at home, London, the UK

Registered midwife, The Royal London Hospital Trust, London, the UK

Midwife at the Royal London Hospital Trust and independent midwife

London, the UK

Volunteer midwife, Muhimbili Hospital, Dar es Salaam, Tanzania

Midwife partner in independent midwives practise, Wageningen,

the Netherlands (Assisted $>1500$ births, $75 \%$ at home)

https://verloskundigenwageningen.nl/

Birth of son Tobias, at home, Wageningen, the Netherlands

Birth of son Benjamin, in hospital, Wageningen, the Netherlands

Treasurer in executive board International Confederation of Midwives

(ICM) https://www.internationalmidwives.org/

Editor Dutch Midwifery journal, Royal Dutch Organisation of Midwives (KNOV), Utrecht, the Netherlands

Chair of Board, Gelderse Vallei regional midwives consortium, the Netherlands

Advisor for the development of Code of Conduct for Domestic violence and child abuse, Royal Dutch Organisation of Midwives (KNOV), Utrecht, the Netherlands

Midwife policy advisor for vulnerable communities (asylum seekers, female genital mutilation, domestic violence, child abuse) Royal Dutch Organisation of Midwives (KNOV), Utrecht, the Netherlands

2009 achterstandssituaties, Stuurgroep zwangerschap en geboorte) Ministry

of Health, the Netherlands. On behalf of Royal Dutch Organisation of Midwives (KNOV), Utrecht, the Netherlands

2010-2013

2010-2016

2013

2013-date

2016

2017

2017-date

2017-date

2020-date

2020

2020
Initiator and Board member midwives4mothers Charity,

https://midwives4mothers.nl/

Policy advisor international midwifery affairs, Royal Dutch Organisation of Midwives (KNOV), Utrecht, the Netherlands

Initiator and developer of the 'Dutch midwifery summer school' a two week program for midwives from abroad to experience the Dutch Midwifery system Royal Dutch Organisation of Midwives (KNOV), Utrecht, the Netherlands

Twinning project manager Sierra Leone/Morocco/the Netherlands

/Ghana/Iceland- Royal Dutch Organisation of Midwives (KNOV) supported by midwives4mothers Charity https://midwives4mothers.nl/

Manager independent midwives practice 'De Bakermat praktijk voo verloskunde', Wageningen, the Netherlands https://midwife.n//

Founding member social enterprise twin to win http://twintowin.com/ and twintowin Web Application (French \& English)

Strategy \& Policy manager, Royal Dutch Organisation of Midwives (KNOV), Utrecht, the Netherlands

President International Confederation of Midwives (ICM)

https://www.internationalmidwives.org/

Board member, Partnership for Maternal, New-born and Child Health (PMNCH) https://www.who.int/pmnch/en/

Board member (treasurer), With Women Charity

https://www.withwomen.net/

Steering Group Humanitarian and Fragile Settings, (PMNCH)

https://www.who.int/pmnch/en/

Doctoral Public Defence, Maastricht University, the Netherlands

Twinning, a promising dynamic process

to strengthen the agency

of midwives 
